\title{
TRANSVERSALS, DUALITY, AND IRRATIONAL ROTATION
}

\author{
ANNA DUWENIG AND HEATH EMERSON
}

\begin{abstract}
An early result of Noncommutative Geometry was Connes' observation in the 1980's that the Dirac-Dolbeault cycle for the 2-torus $\mathbb{T}^{2}$, which induces a Poincaré self-duality for $\mathbb{T}^{2}$, can be 'quantized' to give a spectral triple and a $\mathrm{K}$-homology class in $\mathrm{KK}_{0}\left(A_{\theta} \otimes A_{\theta}, \mathbb{C}\right)$ providing the co-unit for a Poincaré self-duality for the irrational rotation algebra $A_{\theta}$ for any $\theta \in \mathbb{R} \backslash \mathbb{Q}$. Connes' proof, however, relied on a K-theory computation and does not supply a representative cycle for the unit of this duality. Since such representatives are vital in applications of duality, we supply such a cycle in unbounded form in this article. Our approach is to construct, for any non-zero integer $b$, a finitely generated projective module $\mathcal{L}_{b}$ over $A_{\theta} \otimes A_{\theta}$ by using a reduction-toa-transversal argument of Muhly, Renault, and Williams, applied to a pair of Kronecker foliations along lines of slope $\theta$ and $\theta+b$, using the fact that these flows are transverse to each other. We then compute Connes' dual of $\left[\mathcal{L}_{b}\right]$ and prove that we obtain an invertible $\tau_{b} \in \mathrm{KK}_{0}\left(A_{\theta}, A_{\theta}\right)$, represented by an equivariant bundle of Dirac-Schrödinger operators. An application of equivariant Bott Periodicity gives a form of higher index theorem describing functoriality of such ' $b$-twists' and this allows us to describe the unit of Connes' duality in terms of a combination of two constructions in KK-theory. This results in an explicit spectral representative of the unit - a kind of 'quantized Thom class' for the diagonal embedding of the noncommutative torus.
\end{abstract}

\section{INTRODUCTION}

The (irrational) rotation algebra $A_{\theta}$ is the crossed-product $\mathrm{C}^{*}$-algebra $C(\mathbb{T}) \rtimes_{\theta} \mathbb{Z}$ associated to a rotation $z \mapsto e^{2 \pi i \theta} z$ of the circle by an (irrational) angle $\theta$. The complex coordinate $V(z)=z$ on $\mathbb{T}$ and the generator $U$ of the group action in the crossed-product, are a pair of unitaries in $A_{\theta}$ which generate it as a $\mathrm{C}^{*}$-algebra and satisfy the relation

$$
V U=e^{2 \pi i \theta} U V .
$$

In particular, when $\theta=0$ we obtain the commutative $\mathrm{C}^{*}$-algebra $C\left(\mathbb{T}^{2}\right)$ of continuous functions on the 2 -torus, and accordingly $A_{\theta}$ is often called the 'noncommutative torus.'

Compact spin ${ }^{c}$-manifolds such as $\mathbb{T}^{2}$ exhibit duality in KK. Two $\mathrm{C}^{*}$-algebras $A$ and $B$ are dual in $\mathrm{KK}$ if there exists a pair of classes

$$
\Delta \in \mathrm{KK}_{0}(A \otimes B, \mathbb{C}), \quad \widehat{\Delta} \in \mathrm{KK}_{0}(\mathbb{C}, B \otimes A)
$$

satisfying the zig-zag equations:

$$
\left(1_{A} \otimes \widehat{\Delta}\right) \otimes_{A \otimes B \otimes A}\left(\Delta \otimes 1_{A}\right)=1_{A}, \quad\left(\widehat{\Delta} \otimes 1_{B}\right) \otimes_{B \otimes A \otimes B}\left(1_{B} \otimes \Delta\right)=1_{B} .
$$

Received by the editors March 20, 2020, and, in revised form, July 16, 2020.

2020 Mathematics Subject Classification. Primary 19K35; Secondary 58B34, 46L08.

This research was supported by an NSERC Discovery grant. 
We will refer to the class $\widehat{\Delta}$ as the unit, and $\Delta$ as the co-unit of the duality, with reference to the theory of adjoint functors. A cup-cap operation using $\Delta$ determines a map

$$
\Delta \cup_{\sqcup}: \mathrm{KK}_{i}\left(D_{1}, A_{\theta} \otimes D_{2}\right) \cong \mathrm{KK}_{i}\left(A_{\theta} \otimes D_{1}, D_{2}\right),
$$

for any pair $D_{1}, D_{2}$ of separable $\mathrm{C}^{*}$-algebras, and it can be checked that $\widehat{\Delta}$ provides a similar map which inverts it, because of the zig-zag equations.

If $X$ is a compact $\operatorname{spin}^{c}$-manifold, then the diagonal embedding $\delta: X \rightarrow X \times X$ has a normal bundle $\nu$ with canonical K-orientation and a Thom class $\xi \in \mathrm{K}^{-n}(\nu)$. Using a tubular neighbourhood embedding $\nu \subseteq X \times X$, we can extend the Thom cycle and class to zero outside the neighbourhood, yielding a $\mathrm{K}$-theory class for $X \times X$ that is equal by definition to $\widehat{\Delta} \in \mathrm{KK}_{+n}(\mathbb{C}, C(X \times X))$, and which is supported in an arbitrarily small neighbourhood of the diagonal $X \subset X \times X$. This construction determines the unit for a self-duality for $X$.

The co-unit $\Delta \in \mathrm{KK}_{-n}(C(X \times X), \mathbb{C})$ in this duality is represented, analytically, by the Dirac cycle for $X$, consisting of the Dirac operator acting on $L^{2}$-spinors on $X$. This gives a cycle for $\mathrm{KK}_{-n}(C(X), \mathbb{C})$, and pulling it back by the *-homomorphism $C(X \times X) \rightarrow C(X)$ of restriction to the diagonal results in a cycle for $\mathrm{KK}_{-n}(C(X \times X), \mathbb{C})$.

In the 80 's, Connes suggested that there might be $\mathrm{C}^{*}$-algebras which behave in some sense like 'noncommutative manifolds,' and one possible way in which this might happen would be if there were examples of $\mathrm{C}^{*}$-algebras arising in geometric situations which exhibit KK-duality. He pointed out that the Dirac cycle for the 2torus can be adapted slightly so as to give a cycle and class $\Delta_{\theta} \in \mathrm{KK}_{0}\left(A_{\theta} \otimes A_{\theta}, \mathbb{C}\right)$ inducing duality even for the noncommutative $A_{\theta}$ 's (see [3] and [2]). There are now several other examples of $\mathrm{C}^{*}$-algebras with KK-theoretic duality: groupoid $\mathrm{C}^{*}$-algebras arising from hyperbolic dynamical systems ([12] and [13]), crossedproducts by actions of Gromov hyperbolic groups on their boundaries [8], and orbifold $\mathrm{C}^{*}$-algebras [6]. Duality for Cuntz-Pimsner algebras is studied in [25]; in the special case of $A_{\theta}$, they recover Connes' formula for the unit $\widehat{\Delta}_{\theta}$ in terms of known K-theory generators for $A_{\theta}$. In some cases, the Baum-Connes conjecture boils down to a form of duality between a group $\mathrm{C}^{*}$-algebra and its classifying space, and some of these special cases are studied in [22].

Connes' remark about $A_{\theta}$ was that the class $\Delta_{\theta}$ built from the Dolbeault operator on $\mathbb{T}^{2}$ induces a self-duality for $A_{\theta}$ because the induced intersection form

$$
\mathrm{K}_{*}\left(A_{\theta}\right) \times \mathrm{K}_{*}\left(A_{\theta}\right) \longrightarrow \mathrm{K}_{*}\left(A_{\theta} \otimes A_{\theta}\right) \stackrel{\left\langle\cdot, \Delta_{\theta}\right\rangle}{\longrightarrow} \mathbb{Z}
$$

can be computed directly and is non-degenerate. However, Connes' formula for the unit $\widehat{\Delta}_{\theta}$ has no obvious representative cycle. What is desired in a KK-duality is a pair of cycles: one for the K-theory and one for the K-homology of $A_{\theta} \otimes A_{\theta}$ in this case. Cycles lead to applications (for example to 'noncommutative Lefschetz fixed-point formulas' 7].)

The purpose of this article is to describe a geometrically defined spectral (that is, unbounded) cycle for $\mathrm{K}_{0}\left(A_{\theta} \otimes A_{\theta}\right)$ representing $\widehat{\Delta}_{\theta}$. Our method yields a connection between Connes' Dolbeault duality class, and a geometric construction with noncompact transversals, which goes back to ideas in [21].

Let $\mathcal{B}_{\theta}$ and $\mathcal{B}_{\theta+b}$ denote the transformation groupoids corresponding to Kronecker flows on $\mathbb{T}^{2}$ along lines of slope $\theta$ and $\theta+b$. Since $\mathcal{B}_{\theta}$ and $\mathcal{B}_{\theta+b}$ are transverse, the restriction of the groupoid $\mathcal{B}_{\theta} \times \mathcal{B}_{\theta+b}$ to the diagonal $\mathbb{T}^{2}$ in its unit space $\mathbb{T}^{2} \times \mathbb{T}^{2}$ is 
étale. A well-known construction of Muhly, Renault, and Williams [20] provides an explicit strong Morita equivalence between the restricted groupoid and $\mathcal{B}_{\theta} \times \mathcal{B}_{\theta+b}$, and hence with $\left(\mathbb{T} \rtimes_{\theta} \mathbb{Z}\right) \times\left(\mathbb{T} \rtimes_{\theta+b} \mathbb{Z}\right)$, and then with $\left(\mathbb{T} \rtimes_{\theta} \mathbb{Z}\right) \times\left(\mathbb{T} \rtimes_{\theta} \mathbb{Z}\right)$.

We obtain a strong Morita equivalence between the unital $\mathrm{C}^{*}$-algebra of the restricted groupoid and $A_{\theta} \otimes A_{\theta}$. Since the former is étale, the strong Morita equivalence bimodule is finitely generated projective as an $A_{\theta} \otimes A_{\theta}$-module. Let $\left[\mathcal{L}_{b}\right] \in \mathrm{KK}_{0}\left(\mathbb{C}, A_{\theta} \otimes A_{\theta}\right)$ be its class.

We next construct a morphism

$$
\tau_{b} \in \mathrm{KK}_{0}\left(A_{\theta}, A_{\theta}\right)
$$

for any $b \in \mathbb{Z}$, which we call the ' $b$-twist,' and which is represented by applying descent

$$
\mathrm{KK}_{0}^{\mathbb{Z}}(C(\mathbb{T}), C(\mathbb{T})) \rightarrow \mathrm{KK}_{0}\left(C(\mathbb{T}) \rtimes_{\theta} \mathbb{Z}, C(\mathbb{T}) \rtimes_{\theta} \mathbb{Z}\right)=\mathrm{KK}_{0}\left(A_{\theta}, A_{\theta}\right)
$$

to the class of a $\mathbb{Z}$-equivariant bundle of Dirac-Schrödinger operators $\frac{\partial}{\partial r}+r$ over the circle $\mathbb{T}$. The $b$-twist has the features of acting as multiplication by the matrix $\left[\begin{array}{ll}1 & b \\ 0 & 1\end{array}\right]$ on $\mathrm{K}_{0}\left(A_{\theta}\right)$ with the standard identification $\mathrm{K}_{0}\left(A_{\theta}\right) \cong \mathbb{Z}^{2}$, and acting as the identity on $\mathrm{K}_{1}\left(A_{\theta}\right)$. (In particular, $\tau_{b}$ is not represented by any automorphism of $A_{\theta}$.)

In Section 4, using equivariant Bott Periodicity, we prove a kind of index theorem about $b$-twists, to the effect that the family of morphisms $\left\{\tau_{b}\right\}_{b \in \mathbb{Z}}$ form a cyclic group in the invertibles in $\mathrm{KK}_{0}\left(A_{\theta}, A_{\theta}\right)$.

The main result of this article is:

Theorem 1.1. The class $\Delta_{\theta}$ of Connes, and $\widehat{\Delta}_{\theta}:=\left(1_{A_{\theta}} \otimes \tau_{-b}\right)_{*}\left(\left[\mathcal{L}_{b}\right]\right)$ for $b>0$ are the co-unit and unit of a KK-self-duality for $A_{\theta}$.

The description of $\widehat{\Delta}_{\theta}$ given in the theorem leads to an explicit unbounded representative of $\widehat{\Delta}_{\theta}$ in the form of a self-adjoint operator on a Hilbert module a kind of 'quantized' Thom class for the diagonal embedding $\mathbb{T}_{\theta}^{2} \rightarrow \mathbb{T}_{\theta}^{2} \times \mathbb{T}_{\theta}^{2}$. See Theorem 6.5 for the exact statement.

\section{Preliminaries}

2.1. Irrational rotation on the circle. In this paper, we are mainly interested in a class of group actions, but we will use groupoid methods prolifically.

Irrational rotation on the circle $\mathbb{T}$ is given by the $\mathbb{Z}$-action $n \mapsto \alpha_{n}$ where $\alpha_{n}([x])=[x+n \theta],[x] \in \mathbb{T}:=\mathbb{R} / \mathbb{Z}$. The action determines a transformation groupoid $\mathcal{A}_{\theta}:=\mathbb{T} \rtimes_{\theta} \mathbb{Z}$ with composition rules

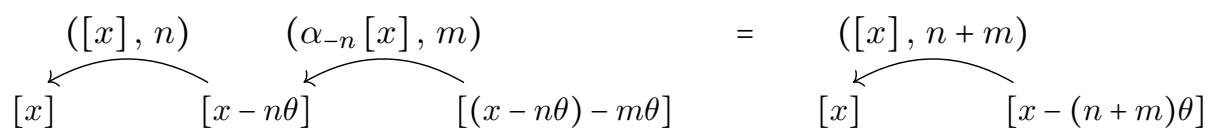

Inverses are given by $([x], n)^{-1}=\left(\alpha_{-n}[x],-n\right)$.

The irrational rotation algebra $A_{\theta}$ is the groupoid $\mathrm{C}^{*}$-algebra of this groupoid. Equivalently, $A_{\theta}$ is the crossed-product

$$
A_{\theta}:=C^{*}\left(\mathcal{A}_{\theta}\right) \cong C(\mathbb{T}) \rtimes_{\theta} \mathbb{Z} .
$$


As is well-known, the irrational rotation algebra is the universal $\mathrm{C}^{*}$-algebra $A_{\theta}$ generated by two unitaries $U, V$ subject to the relation $V U=e^{2 \pi i \theta} U V$. Note that

$$
\mathfrak{A}:=\left\{\sum_{m, n \in \mathbb{Z}} a_{m, n} V^{m} U^{n} \mid\left(a_{m, n}\right)_{m, n} \in \mathcal{S}\left(\mathbb{Z}^{2}\right)\right\}
$$

is a dense subalgebra, where $\left(a_{m, n}\right)_{m, n} \in \mathcal{S}\left(\mathbb{Z}^{2}\right)$ if and only if for all $k \in \mathbb{Z}^{+}$,

$$
\sup _{m, n}\left\{\left(|m|^{k}+|k|^{k}\right)\left|a_{m, n}\right|\right\}<\infty .
$$

In the crossed product picture, $V$ corresponds to the generator of $C(\mathbb{T})$ and $U$ to the generator of $\mathbb{Z}$.

As such, $A_{\theta}$ is sometimes referred to as the noncommutative torus, since the $\mathrm{C}^{*}$ algebra $C\left(\mathbb{T}^{2}\right)$ of continuous functions on the 2 -torus is generated by two commuting unitaries $U, V$ (namely, the coordinate projections).

2.2. Poincaré duality. A KK-theoretic Poincaré duality between two $\mathrm{C}^{*}$-algebras $A$ and $B$, determines an isomorphism between the K-theory groups of $A$ and the K-homology groups of $B$. An important motivating example comes from smooth manifold theory: If $X$ is a smooth compact manifold, then it is a result of Kasparov that $C(X)$ is Poincaré dual to $C_{0}(T X)$, where $T X$ is the tangent bundle. The Poincaré duality isomorphism sends the K-theory class defined by the symbol of an elliptic operator, to the K-homology class of the operator.

If $X$ carries a $\operatorname{spin}^{c}$-structure, i.e. a K-orientation on its tangent bundle, then $C_{0}(T X)$ is KK-equivalent to $C(X)$ by the Thom isomorphism, and so $C(X)$ has a self-duality of a dimension shift of $\operatorname{dim} X$. A basic example is $X=\mathbb{T}^{2}$.

Duality in this sense is an example of an adjunction of functors, and is, like with adjoint functors in general, determined by two classes, usually called the the unit and co-unit, here denoted $\widehat{\Delta}$ and $\Delta$ respectively.

Definition 2.1. We say that two (nuclear, separable, unital) $\mathrm{C}^{*}$-algebras $A, B$ are Poincaré dual (with dimension shift of zero) if there exist $\Delta \in \mathrm{KK}_{0}(A \otimes B, \mathbb{C}$ ) and $\widehat{\Delta} \in \mathrm{KK}_{0}(\mathbb{C}, B \otimes A)$ which satisfy the following so-called zig-zag equations,

$$
\begin{aligned}
& \widehat{\Delta} \otimes_{B} \Delta:=\left(1_{A} \otimes \widehat{\Delta}\right) \otimes_{A \otimes B \otimes A}\left(\Delta \otimes 1_{A}\right)=1_{A} \in \operatorname{KK}_{0}(A, A) \quad \text { and } \\
& \widehat{\Delta} \otimes_{A} \Delta:=\left(\widehat{\Delta} \otimes 1_{B}\right) \otimes_{B \otimes A \otimes B}\left(1_{B} \otimes \Delta\right)=1_{B} \in \operatorname{KK}_{0}(B, B) .
\end{aligned}
$$

We call $(\Delta, \widehat{\Delta})$ (Poincaré) duality pair.

The co-unit $\Delta \in \mathrm{KK}_{0}(A \otimes B, \mathbb{C})$, for example, determines a cup-cap product map

$$
\Delta \cup \cup_{\lrcorner}: K_{*}\left(D_{1}, B \otimes D_{2}\right) \rightarrow \mathrm{KK}_{*}\left(A \otimes D_{1}, D_{2}\right), \quad \Delta \cup f:=\left(1_{A} \otimes_{\mathbb{C}} f\right) \otimes_{A \otimes B} \Delta .
$$

The unit can be similarly used to define a system of maps dual to the above, and some manipulations show that the maps are inverse if the zig-zag equations hold.

There are now a number of examples of Poincaré dual pairs of $\mathrm{C}^{*}$-algebras: see [6], 12, [8], 13. The first noncommutative example, a Poincaré self-duality for the irrational rotation algebra $A_{\theta}$, is due to Connes (see [2]) and is the primary interest of this article.

Although we have not included it in the definition, one hopes to find explicit cycles for the classes $\Delta$ and $\widehat{\Delta}$ in a Poincaré duality. Connes has defined a cycle ([2], p. 604) whose class $\Delta_{\theta} \in \mathrm{KK}_{0}\left(A_{\theta} \otimes A_{\theta}, \mathbb{C}\right)$ determines the duality for $A_{\theta}$ alluded 
to above, but the formula he gave for the dual class $\widehat{\Delta}_{\theta} \in \mathrm{KK}_{0}\left(\mathbb{C}, A_{\theta} \otimes A_{\theta}\right)=$ $\mathrm{K}_{0}\left(A_{\theta} \otimes A_{\theta}\right)$ was of the type $\widehat{\Delta}=x \otimes_{\mathbb{C}} y+x^{\prime} \otimes_{\mathbb{C}} y^{\prime}+\ldots$, where $x, x^{\prime} \in \mathrm{K}_{*}(A)$ and $y, y^{\prime} \in \mathrm{K}_{*}(B)$, and $\otimes_{\mathbb{C}}$ refers to the external product in $\mathrm{KK}$; this does not specify a cycle, but a class. It is this missing cycle, representing $\widehat{\Delta}_{\theta}$, that this article aims to supply.

Connes' class $\Delta_{\theta} \in \mathrm{KK}_{0}\left(A_{\theta} \otimes A_{\theta}, \mathbb{C}\right)$ can be defined as follows.

Lemma 2.2. On $L^{2}:=L^{2}(\mathbb{T} \times \mathbb{Z})$, define

$$
\omega_{1}, \omega_{2}: C(\mathbb{T}) \rightarrow \mathcal{B}\left(L^{2}\right) \quad \text { and } \quad u, v: \mathbb{Z} \rightarrow \mathcal{U}\left(L^{2}\right)
$$

for $f \in C(\mathbb{T}), k \in \mathbb{Z}, \xi \in L^{2}(\mathbb{T}), \mathrm{e}_{n} \in \ell^{2}(\mathbb{Z})$ by

$$
\begin{array}{ccc}
\omega_{1}(f)\left(\xi \otimes \mathrm{e}_{n}\right):=\left(\alpha_{-n}(f) \cdot \xi\right) \otimes \mathrm{e}_{n} & \text { and } & u_{k}\left(\xi \otimes \mathrm{e}_{n}\right):=\xi \otimes \mathrm{e}_{k+n} \\
\omega_{2}(f)\left(\xi \otimes \mathrm{e}_{n}\right):=(f \cdot \xi) \otimes \mathrm{e}_{n} & & v_{k}\left(\xi \otimes \mathrm{e}_{n}\right):=(k \cdot \xi) \otimes \mathrm{e}_{n-k},
\end{array}
$$

where $k . \xi=\xi \circ \alpha_{-k}$ for $\xi$ in the subspace $C(\mathbb{T}) \subseteq L^{2}(\mathbb{T})$.

Then the pairs $\left(\omega_{1}, u\right)$ and $\left(\omega_{2}, v\right)$ are covariant for $(C(\mathbb{T}), \alpha, \mathbb{Z})$ and hence induce representations of $A_{\theta}$ on $L^{2}$. Moreover, these two representations commute and thus give a representation $\pi$ of $A_{\theta} \otimes A_{\theta}$ on $L^{2}$, so we obtain an unbounded cycle

$$
\left(L^{2} \oplus L^{2}, \pi \oplus \pi, d_{\Delta}\right) \text { for } \mathrm{KK}_{0}\left(A_{\theta} \otimes A_{\theta}, \mathbb{C}\right),
$$

where

$$
d_{\Delta}:=\left[\begin{array}{cc}
0 & D_{\mathbb{Z}}-i D_{\mathbb{T}} \\
D_{\mathbb{Z}}+i D_{\mathbb{T}} & 0
\end{array}\right]
$$

with

$$
D_{\mathbb{Z}}=2 \pi \mathrm{M}^{\mathbb{Z}} \text { and } D_{\mathbb{T}}=-i \frac{\partial}{\partial \Theta} \text {, i.e. }\left(D_{\mathbb{Z}} \pm i D_{\mathbb{T}}\right)\left(z^{m} \otimes \mathrm{e}_{n}\right)=2 \pi(n \pm i m) \cdot z^{m} \otimes \mathrm{e}_{n} .
$$

The operator $d_{\Delta}$ is, more precisely, the closure of the corresponding essentially self-adjoint operator with essential domain two copies of the Schwartz space

$$
\left\{\sum_{m, n \in \mathbb{Z}} a_{m, n} z^{m} \otimes \mathrm{e}_{n} \in L^{2}(\mathbb{T}) \otimes l^{2}(\mathbb{Z}) \mid\left(a_{m, n}\right)_{m, n} \in \mathcal{S}\left(\mathbb{Z}^{2}\right)\right\} .
$$

For the definition of $\mathcal{S}\left(\mathbb{Z}^{2}\right)$, see Equation (2.1).

Definition 2.3. We let $\Delta_{\theta} \in \mathrm{KK}_{0}\left(A_{\theta} \otimes A_{\theta}, \mathbb{C}\right)$ be the class of the cycle described in Lemma 2.2

\section{Pairs of transverse Kronecker flows}

The Kronecker flow on the 2 -torus $\mathbb{T}^{2}$ for angle $\theta$ is given by the $\mathbb{R}$-action on $\mathbb{T}^{2}=\mathbb{R}^{2} / \mathbb{Z}^{2}$ defined by

$$
\beta_{t}\left[\begin{array}{l}
x \\
y
\end{array}\right]=\left[\begin{array}{c}
x+t \theta \\
y+t
\end{array}\right] .
$$

The corresponding transformation groupoid $\mathcal{B}_{\theta}:=\mathbb{T}^{2} \rtimes_{\theta} \mathbb{R}$ is defined as:
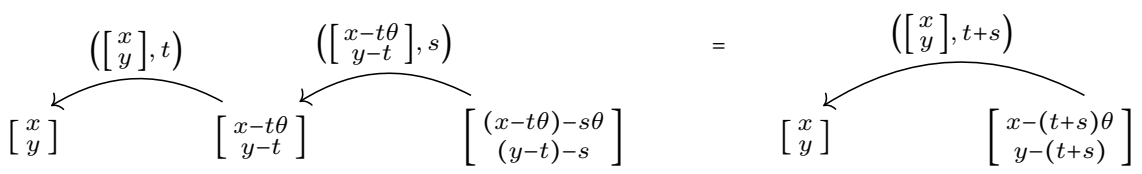
In particular, $\left(\left[\begin{array}{l}x \\ y\end{array}\right], t\right)^{-1}=\left(\left[\begin{array}{c}x-t \theta \\ y-t\end{array}\right],-t\right)$. We denote the momentum maps of $\mathcal{B}_{\theta}$ by $s_{\theta}$ and $r_{\theta}$. Orbits of the Kronecker flow are lines $\vec{x}+t\left[\begin{array}{l}\theta \\ 1\end{array}\right]$ in the 2 -torus $\mathbb{T}^{2}$. If

$$
X:=\left\{t\left[\begin{array}{l}
1 \\
0
\end{array}\right] \mid t \in \mathbb{R}\right\} \subseteq \mathbb{T}^{2}=\left(\mathcal{B}_{\theta}\right)^{(0)}
$$

denotes the $x$-axis, then the associated reduction groupoid,

$$
\mathcal{R}_{\theta}:=s_{\theta}^{-1}(X) \cap r_{\theta}^{-1}(X) \subseteq \mathcal{B}_{\theta},
$$

is isomorphic to $\mathcal{A}_{\theta}$ : an element $\left(\left[\begin{array}{l}x \\ y\end{array}\right], s\right)$ is in $\mathcal{R}_{\theta}$ if and only if $[y]=[0]$ and $s \in \mathbb{Z}$, and the map

$$
\begin{gathered}
\mathcal{R}_{\theta} \longrightarrow \mathbb{T} \times \mathbb{Z} \\
\left(\left[\begin{array}{l}
x \\
y
\end{array}\right], s\right) \longmapsto([x], s)
\end{gathered}
$$

is a groupoid isomorphism between $\mathcal{R}_{\theta}$ and $\mathcal{A}_{\theta}$.

In particular, since $X$ is closed and meets every orbit, and since the restriction of $\mathcal{B}_{\theta}$ 's range and source maps to $s_{\theta}^{-1}(X)$ and to $r_{\theta}^{-1}(X)$ are open maps onto their image, Example 2.7 in [20] implies that we have an equivalence $\mathcal{X}_{\theta}$ of groupoids,

$$
\mathcal{X}_{\theta}: \quad \mathcal{B}_{\theta} \curvearrowright s_{\theta}^{-1}(X) \curvearrowleft \mathcal{A}_{\theta} .
$$

Instead of reducing $\mathcal{B}_{\theta}$ to its $x$-axis, we could have reduced to a line $t\left[\begin{array}{c}q \\ -p\end{array}\right]$ of slope $\frac{-p}{q}$ for $p, q$ relatively prime, in which case we would have gotten an equivalence between $\mathcal{B}_{\theta}$ and $\mathcal{A}_{M(\theta)}$ where

$$
M(\theta)=\frac{m \theta+n}{p \theta+q} \text { for } M=\left[\begin{array}{cc}
m & n \\
p & q
\end{array}\right] \in \mathrm{SL}_{2}(\mathbb{Z})
$$

is the Möbius transform of $\theta$. An alternative approach is to change the slope on the foliated torus instead of the rotational angle on the circle, using the following:

Lemma 3.1. For any $M=\left[\begin{array}{cc}m & n \\ p & q\end{array}\right]$ in $\mathrm{GL}_{2}(\mathbb{Z})$, the transformation groupoids $\mathcal{B}_{\theta}$ and $\mathcal{B}_{M(\theta)}$ are isomorphic via

$$
\begin{aligned}
\varphi_{\theta}^{M}: \quad \mathcal{B}_{\theta} & \longrightarrow \mathcal{B}_{M(\theta)} \\
\left(\left[\begin{array}{l}
x \\
y
\end{array}\right], t\right) & \longmapsto\left(M\left[\begin{array}{l}
x \\
y
\end{array}\right], t(p \theta+q)\right)
\end{aligned}
$$

Note that $\varphi_{M(\theta)}^{N} \circ \varphi_{\theta}^{M}=\varphi_{\theta}^{N M}$ for $N$ another such matrix and $\varphi_{\theta}^{\mathbb{1}_{2}}=\mathrm{id}_{\mathcal{B}_{\theta}}$. Further, even though $M(\theta)=(-M)(\theta)$, we should note that $\varphi_{\theta}^{M} \neq \varphi_{\theta}^{-M}$.

Definition 3.2. Let $\mathcal{X}_{\theta}^{M}$ be the $\left(\mathcal{B}_{M(\theta)}, \mathcal{A}_{\theta}\right)$-equivalence constructed out of $\mathcal{X}_{\theta}$ via $\varphi_{\theta}^{M}$.

Given two matrices $M, N \in \mathrm{GL}_{2}(\mathbb{Z})$, then $\mathcal{X}_{\theta}^{M} \times \mathcal{X}_{\theta}^{N}$ is a groupoid equivalence between $\mathcal{B}_{M(\theta)} \times \mathcal{B}_{N(\theta)}$ and $\mathcal{A}_{\theta} \times \mathcal{A}_{\theta}$. Moreover, if $M(\theta) \neq N(\theta)$, the diagonal

$$
D_{M, N}:=\left\{[x, y, x, y] \mid[x, y] \in \mathbb{T}^{2}\right\} \subseteq \mathbb{T}^{2} \times \mathbb{T}^{2}=\left(\mathcal{B}_{M(\theta)} \times \mathcal{B}_{N(\theta)}\right)^{(0)}
$$

meets every orbit. Hence, $\mathcal{B}_{M(\theta)} \times \mathcal{B}_{N(\theta)}$ is equivalent to the reduction groupoid

$$
\mathcal{D}_{M, N}:=\left(\mathcal{B}_{M(\theta)} \times \mathcal{B}_{N(\theta)}\right)_{D_{M, N}}^{D_{M, N}}
$$

via $r_{\theta}^{-1}\left(D_{M, N}\right)$, and all in all we have the following chain of equivalences:

$$
\mathcal{D}_{M, N} \curvearrowright r_{\theta}^{-1}\left(D_{M, N}\right) \curvearrowleft \mathcal{B}_{M(\theta)} \times \mathcal{B}_{N(\theta)} \curvearrowright \mathcal{X}_{\theta}^{M} \times \mathcal{X}_{\theta}^{N} \curvearrowleft \mathcal{A}_{\theta} \times \mathcal{A}_{\theta} .
$$

Thus, we can construct a Morita equivalence from the $\mathrm{C}^{*}$-algebra of $\mathcal{D}_{M, N}$ to $A_{\theta} \otimes A_{\theta}$. It will turn out that $\mathcal{D}_{M, N}$ is an étale groupoid with compact unit space, 
so its $\mathrm{C}^{*}$-algebra is unital, and the Morita equivalence is actually a right f.g.p. module over $A_{\theta} \otimes A_{\theta}$, i.e. corresponds to a K-theory class.

While this description of the K-theory class is nice and geometric, we will try to find an easier one. To this end, consider the following diagram:

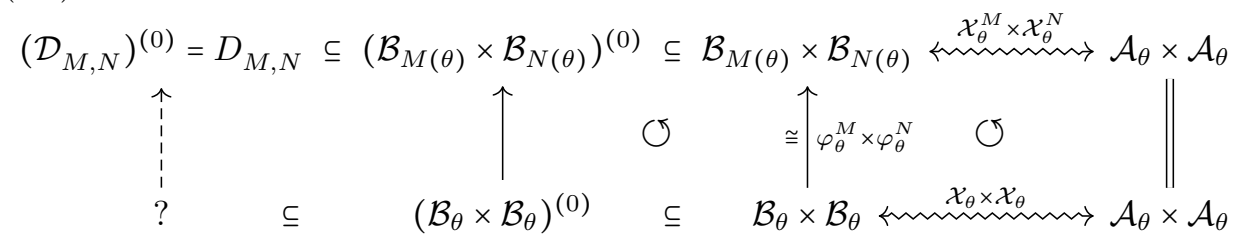

The right-hand square of the diagram commutes since, by definition, the map $\varphi_{\theta}^{M} \times \varphi_{\theta}^{N}$ turns the equivalence $\mathcal{X}_{\theta} \times \mathcal{X}_{\theta}$ into the equivalences $\mathcal{X}_{\theta}^{M} \times \mathcal{X}_{\theta}^{N}$. The middle square commutes since $\varphi_{\theta}^{M} \times \varphi_{\theta}^{N}$ is a groupoid isomorphism, i.e. it maps unit space to unit space. We want to fill in the bottom left to make the left-hand square commute as well. In other words, the question mark represents the preimage of $D_{M, N}$ under $\varphi_{\theta}^{M} \times \varphi_{\theta}^{N}$, which we compute to be

$$
\left(\varphi_{M^{-1}}^{M(\theta)} \times \varphi_{N^{-1}}^{N(\theta)}\right)\left(D_{M, N}\right)=\left\{\left(M^{-1}\left[\begin{array}{l}
x \\
y
\end{array}\right], 0, N^{-1}\left[\begin{array}{l}
x \\
y
\end{array}\right], 0\right) \mid\left[\begin{array}{l}
x \\
y
\end{array}\right] \in \mathbb{T}^{2}\right\} .
$$

This justifies denoting this subset of $\left(\mathcal{B}_{\theta} \times \mathcal{B}_{\theta}\right)^{(0)}$ by $F_{g}$ for $g:=N^{-1} M$. As far as K-theory is concerned, the f.g.p. $A_{\theta} \otimes A_{\theta}$-module constructed out of the bottom row of Diagram (3.4),

$$
\mathcal{F}_{g}:=r_{\theta}^{-1}\left(F_{g}\right) \cap s_{\theta}^{-1}\left(F_{g}\right) \curvearrowright r_{\theta}^{-1}\left(F_{g}\right) \curvearrowleft \mathcal{B}_{\theta} \times \mathcal{B}_{\theta} \curvearrowright \mathcal{X}_{\theta} \times \mathcal{X}_{\theta} \curvearrowleft \mathcal{A}_{\theta} \times \mathcal{A}_{\theta}=: \mathcal{A},
$$

is the same as the module constructed from the top row,

$\mathcal{D}_{M, N}=r_{\theta}^{-1}\left(D_{M, N}\right) \cap s_{\theta}^{-1}\left(D_{M, N}\right) \curvearrowright r_{\theta}^{-1}\left(D_{M, N}\right) \curvearrowleft \mathcal{B}_{M(\theta)} \times \mathcal{B}_{N(\theta)} \curvearrowright \mathcal{X}_{\theta}^{M} \times \mathcal{X}_{\theta}^{N} \curvearrowleft \mathcal{A}$,

by commutativity of the diagram, and since the induced $\mathrm{C}^{*}$-isomorphism between the $\mathrm{C}^{*}$-algebras of $\mathcal{D}_{M, N}$ and $\mathcal{F}_{g}$ is unital. The clear advantage of considering $\mathcal{F}_{g}$ instead of $\mathcal{D}_{M, N}$ is that we only have to deal with the matrix $g=N^{-1} M$, and not with all 8 entries of $M$ and $N$. The inequality $M(\theta) \neq N(\theta)$ (i.e. $g(\theta) \neq \theta$ ), which we needed to construct $\mathcal{D}_{M, N}$, can be rephrased to

$$
\mu(g):=(a \theta+b)-(c \theta+d) \theta \neq 0 \quad \text { where } g=\left[\begin{array}{ll}
a & b \\
c & d
\end{array}\right] .
$$

We can construct the equivalence between $\mathcal{F}_{g}$ and $\mathcal{A}=\mathcal{A}_{\theta} \times \mathcal{A}_{\theta}$ using $\mathcal{Y}_{g}:=$ $r_{\theta}^{-1}\left(F_{g}\right)$ and $\mathcal{X}:=\mathcal{X}_{\theta} \times \mathcal{X}_{\theta}$ as

$$
\mathcal{F}_{g} \curvearrowright \mathcal{Y}_{g} \star_{\mathcal{B}} \mathcal{X} \curvearrowleft \mathcal{A}
$$

c.f. Proposition 5.1 for the details in the case where $g$ is upper triangular. This equips $C_{c}\left(\mathcal{Y}_{g} *_{\mathcal{B}} \mathcal{X}\right)$ with a $C_{c}\left(\mathcal{F}_{g}\right)-C_{c}(\mathcal{A})$ pre-imprimitivity bimodule structure, which can be completed to a $C^{*}\left(\mathcal{F}_{g}\right)-C^{*}(\mathcal{A})$ Morita equivalence bimodule we call $\mathrm{Z}_{g}$.

Lemma 3.3. Let $X$ be the $x$-axis in $\mathbb{T}^{2}=\left(\mathcal{B}_{\theta}\right)^{(0)}$ as in Equation (3.1). If we use the bijection

$$
\begin{gathered}
s_{\theta}^{-1}(X) \longrightarrow \mathbb{T} \times \mathbb{R} \\
\left(\left[\begin{array}{l}
x \\
y
\end{array}\right], s\right) \longmapsto([x-s \theta], s) \\
\left(\left[\begin{array}{c}
x+s \theta \\
s
\end{array}\right], s\right) \longleftrightarrow([x], s)
\end{gathered}
$$


to identify $\mathcal{X}_{\theta}$ with $\mathbb{T} \times \mathbb{R}$, then $\mathcal{X}_{\theta}$ has the following actions by $\mathcal{B}_{\theta}$ and $\mathcal{A}_{\theta}$ :

$$
\begin{array}{ll}
\mathcal{B}_{\theta} \curvearrowright \mathcal{X}_{\theta}: & \left(\left[\begin{array}{c}
x+(s+r) \theta \\
s+r
\end{array}\right], r\right) \cdot([x], s)=([x], r+s) \\
\mathcal{X}_{\theta} \curvearrowleft \mathcal{A}_{\theta}: & ([x], s) \cdot([x], k)=([x-k \theta], s+k)
\end{array}
$$

where we used the map from Equation (3.2) to identify $s_{\theta}^{-1}(X) \cap r_{\theta}^{-1}(X) \cong \mathcal{A}_{\theta}$.

The proof is straight forward. Let us next describe $\mathcal{F}_{g}$ : one checks

$$
r_{\theta}^{-1}\left(F_{g}\right)=\left\{\left(\left[\begin{array}{l}
x \\
y
\end{array}\right], t_{1}, g\left[\begin{array}{l}
x \\
y
\end{array}\right], t_{2}\right) \mid\left[\begin{array}{l}
x \\
y
\end{array}\right] \in \mathbb{T}^{2}, t_{1}, t_{2} \in \mathbb{R}\right\}
$$

and thus

$$
\mathcal{F}_{g}=\left\{\left(\left[\begin{array}{l}
x \\
y
\end{array}\right], \frac{k+l \theta}{\mu(g)}, g\left[\begin{array}{l}
x \\
y
\end{array}\right], \frac{k(c \theta+d)+l(a \theta+b)}{\mu(g)}\right) \mid k, l \in \mathbb{Z}\right\},
$$

where $\mu(g)$ is as in Equation (3.6). In the following, we will write $\left[\begin{array}{l}x \\ y\end{array}\right]+t\left(\begin{array}{l}\theta \\ 1\end{array}\right):=$ $\left[\begin{array}{c}x+t \theta \\ y+t\end{array}\right]$.

Lemma 3.4. The groupoid $\mathcal{F}_{g}$ is isomorphic to the transformation groupoid of the following $\mathbb{Z}^{2}$ action on $\mathbb{T}^{2}$ :

$$
\mathbb{T}^{2} \curvearrowleft \mathbb{Z}^{2}: \quad\left[\begin{array}{l}
x \\
y
\end{array}\right] \cdot(k, l)=\left[\begin{array}{l}
x \\
y
\end{array}\right]+\frac{k+l \theta}{\mu(g)}\left(\begin{array}{l}
\theta \\
1
\end{array}\right)
$$

In particular, $\mathcal{F}_{g}$ is étale with compact unit space and its $C^{*}$-algebra $C^{*}\left(\mathcal{F}_{g}\right)$ is therefore unital.

Proof. The map

$$
\begin{aligned}
\mathcal{F}_{g}=r_{\theta}^{-1}\left(F_{g}\right) \cap s^{-1}\left(F_{g}\right) & \longrightarrow \mathbb{T}^{2} \rtimes \mathbb{Z}^{2} \\
\left(\left[\begin{array}{l}
x \\
y
\end{array}\right], \frac{k+l \theta}{\mu(g)}, g\left[\begin{array}{l}
x \\
y
\end{array}\right], \frac{k(c \theta+d)+l(a \theta+b)}{\mu(g)}\right) & \longmapsto\left(\left[\begin{array}{l}
x \\
y
\end{array}\right], k, l\right)
\end{aligned}
$$

is an isomorphism of groupoids, where the right-hand side is the alleged transformation groupoid. In particular, the unit space of $\mathcal{F}_{g}$ is $\mathbb{T}^{2}$ and hence compact.

Since $\mathbb{Z}^{2}$ is discrete, the transformation groupoid is étale, and so its unit space is clopen. Its characteristic function is hence a continuous, compactly supported function on $\mathcal{F}_{g}$ and serves as unit in $C^{*}\left(\mathcal{F}_{g}\right)$.

Corollary 3.5. The bimodule $\mathrm{Z}_{g}$ is finitely generated projective as a right $C^{*}(\mathcal{A})$ module, so $\mathcal{L}_{g}=\iota^{*}\left(\mathrm{Z}_{g}\right)$ defines a class in $\operatorname{KK}\left(\mathbb{C}, C^{*}(\mathcal{A})\right)$ where $\iota: \mathbb{C} \rightarrow C^{*}\left(\mathcal{F}_{g}\right)$ is the unique unital map.

Proof. We have seen that $C^{*}\left(\mathcal{F}_{g}\right)$, which acts by compact operators on the Morita bimodule $\mathrm{Z}_{g}$, is unital. Therefore, the operator $\operatorname{id}_{\mathrm{Z}_{g}}$ is $C^{*}(\mathcal{A})$-compact, which means $Z_{g}$ is f.g.p. by [10, Proposition 3.9].

Definition 3.6. We let

$$
\left[\mathcal{L}_{g}\right]:=\iota^{*}\left(\mathrm{Z}_{g}\right) \in \mathrm{KK}_{0}\left(\mathbb{C}, C^{*}(\mathcal{A})\right)=\mathrm{KK}_{0}\left(\mathbb{C}, A_{\theta} \otimes A_{\theta}\right)
$$

be the class of the finitely generated projective right $C^{*}(\mathcal{A})$-module constructed from any $g \in \mathrm{SL}_{2}(\mathbb{Z})$ satisfying Equation (3.6). For $g=\left[\begin{array}{ll}1 & b \\ 0 & 1\end{array}\right]$ with $b \in \mathbb{Z} \backslash\{0\}$, we write $\mathcal{L}_{b}$ instead of $\mathcal{L}_{g}$.

We will use the following lemmas in the arguments to follow. 
Lemma 3.7. If we use the bijection

$$
\begin{gathered}
\mathcal{Y}_{g}=r_{\theta}^{-1}\left(F_{g}\right) \longrightarrow \mathbb{R}^{2} \times \mathbb{T}^{2} \\
\left(\left[\begin{array}{l}
x \\
y
\end{array}\right], t_{1}, g\left[\begin{array}{l}
x \\
y
\end{array}\right], t_{2}\right) \longmapsto\left(t_{1}, t_{2},\left[\begin{array}{l}
x \\
y
\end{array}\right]\right)
\end{gathered}
$$

to identify $\mathcal{Y}_{g} \cong \mathbb{R}^{2} \times \mathbb{T}^{2}$, then the right action by $\mathcal{B}:=\mathcal{B}_{\theta} \times \mathcal{B}_{\theta}$ on an element $\left(t_{1}, t_{2},\left[\begin{array}{l}x \\ y\end{array}\right]\right) \in \mathcal{Y}_{g}$ is given by

$$
\left(t_{1}, t_{2},\left[\begin{array}{l}
x \\
y
\end{array}\right]\right) \cdot\left(\left[\begin{array}{l}
x \\
y
\end{array}\right]-t_{1}\left(\begin{array}{l}
\theta \\
1
\end{array}\right), r_{1}, g\left[\begin{array}{l}
x \\
y
\end{array}\right]-t_{2}\left(\begin{array}{l}
\theta \\
1
\end{array}\right), r_{2}\right)=\left(t_{1}+r_{1}, t_{2}+r_{2},\left[\begin{array}{l}
x \\
y
\end{array}\right]\right) .
$$

If we further use the bijection in Equation (3.7) to identify $\mathcal{F}_{g} \cong \mathbb{T}^{2} \rtimes \mathbb{Z}^{2}$, then the left action of $\mathcal{F}_{g}$ on $\mathcal{Y}_{g}$ is given by

$$
\left(\left[\begin{array}{l}
x \\
y
\end{array}\right]+\frac{k+l \theta}{\mu(g)}\left(\begin{array}{l}
\theta \\
1
\end{array}\right), k, l\right) \cdot\left(t_{1}, t_{2},\left[\begin{array}{l}
x \\
y
\end{array}\right]\right)=\left(\frac{k+l \theta}{\mu(g)}+t_{1}, \frac{k(c \theta+d)+l(a \theta+b)}{\mu(g)}+t_{2},\left[\begin{array}{l}
x \\
y
\end{array}\right]+\frac{k+l \theta}{\mu(g)}\left(\begin{array}{l}
\theta \\
1
\end{array}\right)\right) \text {. }
$$

Elements of $\mathcal{Y}_{g} * \mathcal{X}$, where $\mathcal{X}=\mathcal{X}_{\theta} \times \mathcal{X}_{\theta}$ as before, are given by those $\left(t_{1}, t_{2},[x, y],[v]\right.$, $\left.s_{1},[w], s_{2}\right)$ in $\left(\mathbb{R}^{2} \times \mathbb{T}^{2}\right) \times(\mathbb{T} \times \mathbb{R} \times \mathbb{T} \times \mathbb{R})$ which satisfy

$$
\begin{aligned}
& s_{\mathcal{Y}}\left(t_{1}, t_{2},[x, y]\right)=r_{\mathcal{X}}\left([v], s_{1},[w], s_{2}\right) \\
\Longleftrightarrow & {[x, y]-t_{1}[\theta, 1]=[v, 0]+s_{1}[\theta, 1] \text { and } g[x, y]-t_{2}[\theta, 1]=[w, 0]+s_{2}[\theta, 1] . }
\end{aligned}
$$

In other words,

$$
\left[\begin{array}{l}
x \\
y
\end{array}\right]=\left[\begin{array}{l}
v \\
0
\end{array}\right]+\left(s_{1}+t_{1}\right)\left(\begin{array}{l}
\theta \\
1
\end{array}\right)=g^{-1}\left(\left[\begin{array}{c}
w \\
0
\end{array}\right]+\left(s_{2}+t_{2}\right)\left(\begin{array}{l}
\theta \\
1
\end{array}\right)\right) .
$$

Now, in the balanced $\mathcal{Y}_{g} *_{\mathcal{B}} \mathcal{X}$, we identify the following elements of $\mathcal{Y}_{g} * \mathcal{X}$ :

$$
\left(t_{1}, t_{2},[x, y],[v], s_{1},[w], s_{2}\right) \sim\left(t_{1}+t_{1}^{\prime}, t_{2}+t_{2}^{\prime},[x, y],[v], s_{1}-t_{1}^{\prime},[w], s_{2}-t_{2}^{\prime}\right)
$$

for any $t_{1}^{\prime}, t_{2}^{\prime} \in \mathbb{R}$. We conclude:

Lemma 3.8. If we let

$$
\mathcal{Z}_{g}:=\left\{\left(r_{1}, r_{2},\left[\begin{array}{l}
v \\
w
\end{array}\right]\right) \in \mathbb{R}^{2} \times \mathbb{T}^{2} \mid g\left(\left[\begin{array}{l}
v \\
0
\end{array}\right]+r_{1}\left(\begin{array}{c}
\theta \\
1
\end{array}\right)\right)=\left[\begin{array}{c}
w \\
0
\end{array}\right]+r_{2}\left(\begin{array}{l}
\theta \\
1
\end{array}\right)\right\},
$$

then the following are mutually inverse bijections:

$$
\begin{gathered}
\mathcal{Y}_{g} \star_{\mathcal{B}} \mathcal{X} \longrightarrow \mathcal{Z}_{g} \\
{\left[t_{1}, t_{2},[x, y],[v], s_{1},[w], s_{2}\right] \mapsto\left(t_{1}+s_{1}, t_{2}+s_{2},\left[\begin{array}{c}
v \\
w
\end{array}\right]\right)} \\
{\left[r_{1}, r_{2},\left[v+r_{1} \theta, r_{1}\right][v], 0,[w], 0\right] \longleftrightarrow\left(r_{1}, r_{2},\left[\begin{array}{c}
v \\
w
\end{array}\right]\right)}
\end{gathered}
$$

\section{THE B-TWIST}

Connes' cycle (Definition 2.3 and prior discussion) and corresponding class $\Delta_{\theta} \epsilon$ $\mathrm{KK}_{0}\left(A_{\theta} \otimes A_{\theta}, \mathbb{C}\right)$ is the co-unit of the duality we are going to establish. By the general mechanics of $\mathrm{KK}$, the class $\Delta_{\theta}$ determines a map

$$
\Delta_{\theta} \cup{ }_{\sqcup}: \mathrm{KK}_{0}\left(\mathbb{C}, A_{\theta} \otimes A_{\theta}\right) \rightarrow \mathrm{KK}_{0}\left(A_{\theta}, A_{\theta}\right), f \mapsto\left(f \otimes 1_{A_{\theta}}\right) \otimes_{A_{\theta}^{3}}\left(\Delta_{\theta} \otimes 1_{A_{\theta}}\right),
$$

and the first zig-zag equation asserts that, if $f \in \mathrm{KK}_{0}\left(\mathbb{C}, A_{\theta} \otimes A_{\theta}\right)$ is the unit for a duality with co-unit $\Delta_{\theta}$, then $\Delta_{\theta} \cup f=1_{A_{\theta}}$.

We are going to show in this article that

$$
\Delta_{\theta} \cup\left[\mathcal{L}_{b}\right]=\tau_{b},
$$

where $\left[\mathcal{L}_{b}\right]=\left[\mathcal{L}_{g}\right] \in \mathrm{KK}_{0}\left(\mathbb{C}, A_{\theta} \otimes A_{\theta}\right)$ is the class of the finitely generated projective $A_{\theta} \otimes A_{\theta}$-module constructed in the last section from the transversals for $g=\left[\begin{array}{ll}1 & b \\ 0 & 1\end{array}\right]$ upper triangular and non-trivial, and $\tau_{b}$ is a certain invertible in $\mathrm{KK}_{0}\left(A_{\theta}, A_{\theta}\right)$ which we describe explicitly first. 
Let $b \in \mathbb{Z}$ be any integer. Equip $C_{c}(\mathbb{T} \times \mathbb{R})$ with the following structure:

$$
\begin{aligned}
\phi, \psi \in C_{c}(\mathbb{T} \times \mathbb{R}): & \left\langle\phi_{1} \mid \phi_{2}\right\rangle_{C(\mathbb{T})}([x]) & =\int_{\mathbb{R}} \overline{\phi_{1}} \phi_{2}([x], r) \mathrm{d} r, \\
\mathbb{Z} \curvearrowright C_{c}(\mathbb{T} \times \mathbb{R}): & (l \bullet \phi)([x], r) & =\phi([x-l \theta], r-l), \\
C(\mathbb{T}) \curvearrowright C_{c}(\mathbb{T} \times \mathbb{R}): & (f \bullet \phi)([x], r) & =f([x+r b]) \phi([x], r), \\
C_{c}(\mathbb{T} \times \mathbb{R}) \curvearrowleft C(\mathbb{T}): & (\phi \bullet f)([x], r) & =\phi([x], r) f([x]) .
\end{aligned}
$$

Let $H_{b}^{ \pm}$be the completion of $C_{c}(\mathbb{T} \times \mathbb{R})$ with respect to the pre-inner product given above and set $H_{b}:=H_{b}^{+} \oplus H_{b}^{-}$. For $\lambda \in \mathbb{R}^{\times}$, let $d_{\lambda,+}$ be the closure of the following essentially self-adjoint Dirac-Schrödinger operator on $L^{2}(\mathbb{R})$ with essential domain the Schwartz functions $\mathcal{S}(\mathbb{R})$ on $\mathbb{R}$ :

$$
d_{\lambda,+}:=\lambda \mathrm{M}+\frac{\partial}{\partial r} \quad \text { with adjoint } \quad d_{\lambda,-}:=\lambda \mathrm{M}-\frac{\partial}{\partial r} .
$$

Here, $\mathrm{M}$ is the operator that multiplies by the input of the $\mathbb{R}$-component.

If $A$ and $B$ are $\mathbb{Z}$-C*-algebras, we will denote by $\Psi_{*}^{\mathbb{Z}}(A, B)$ the $\mathbb{Z}$-equivariant unbounded cycles for $\mathrm{KK}_{*}^{\mathbb{Z}}(A, B)$ in the sense of [24, Definition 2.11]. Our KKautomorphism $\tau_{b}$ of $A_{\theta}$ will be obtained by applying Kasparov's descent map

$$
j: \mathrm{KK}_{0}^{\mathbb{Z}}(C(\mathbb{T}), C(\mathbb{T})) \rightarrow \mathrm{KK}_{0}\left(A_{\theta}, A_{\theta}\right) .
$$

By [24, Proposition 2.12], the descent construction of Kasparov adapts to one at the level of unbounded cycles, giving a map

$$
\Psi_{0}^{\mathbb{Z}}(C(\mathbb{T}), C(\mathbb{T})) \rightarrow \Psi_{0}\left(A_{\theta}, A_{\theta}\right),
$$

which, by a slight abuse of notation, we will also denote by $j$. Utilizing it, we will obtain an unbounded cycle representing $\tau_{b}$, which is easier to compute with.

Theorem 4.1. If $\mathbb{Z}$ acts by rotation on $\mathbb{T}$, and $\lambda \in \mathbb{R}^{\times}$, then the pair $\left(H_{b}, \operatorname{id}_{C(\mathbb{T})} \otimes\right.$ $\left.d_{\lambda}\right)$ with

$$
d_{\lambda}:=\left[\begin{array}{cc}
0 & d_{\lambda,-} \\
d_{\lambda,+} & 0
\end{array}\right]
$$

is a cycle in $\Psi_{0}^{\mathbb{Z}}(C(\mathbb{T}), C(\mathbb{T}))$.

Recall that $\operatorname{id}_{C(\mathbb{T})} \otimes d_{\lambda}$ denotes the closure of the operator $\operatorname{id}_{C(\mathbb{T})} \odot d_{\lambda}$ and that the latter's domain contains the dense subspace $C(\mathbb{T}) \odot \mathcal{S}(\mathbb{R})$. The proof proceeds through the following two lemmas.

Lemma 4.2. The operator $\operatorname{id}_{C(\mathbb{T})} \otimes d_{\lambda}$ is odd, self-adjoint, regular, and has compact resolvent.

Note that this in particular implies that $\operatorname{id}_{C(\mathbb{T})} \otimes d_{\lambda}$ is linear with respect to the right $C(\mathbb{T})$-action.

Proof. By construction, $\operatorname{id}_{C(\mathbb{T})} \odot d_{\lambda}$ is odd and symmetric. We compute

$$
d_{\lambda}^{2}=\left[\begin{array}{cc}
\lambda^{2} \mathrm{M}^{2}-\frac{\partial^{2}}{\partial r^{2}}-\lambda & 0 \\
0 & \lambda^{2} \mathrm{M}^{2}-\frac{\partial^{2}}{\partial r^{2}}+\lambda
\end{array}\right],
$$

Consider the $L^{2}$-normalized functions

$$
\psi_{0}(r)=|\lambda|^{\frac{1}{2}} \pi^{\frac{1}{4}} \mathrm{e}^{-|\lambda| \frac{r^{2}}{2}} \quad \text { and } \quad \psi_{l}=(2 l|\lambda|)^{-\frac{1}{2}} \cdot\left(|\lambda| \mathrm{M}-\frac{\partial}{\partial r}\right) \psi_{l-1} .
$$

Note that $\psi_{0}$ is a Schwartz function, i.e. in the domain of $d_{\lambda, \pm}$, and therefore so are all $\psi_{l}$. Moreover, they span a dense subspace of $L^{2}(\mathbb{R})([26$, Proposition 9.8]) and 
they are eigenfunctions of $\lambda^{2} \mathrm{M}^{2}-\frac{\partial^{2}}{\partial r^{2}}([26$, Lemma 9.6]) with corresponding set of eigenvalues

$$
\{(2 l+1)|\lambda|: l=1,2, \ldots\} .
$$

We conclude that the operator $d_{\lambda}^{2}+1$ has the eigenfuctions $\psi_{l} \oplus 0$ and $0 \oplus \psi_{l}$. Thus, the orthonormal basis $\left\{\psi_{l} \oplus 0,0 \oplus \psi_{l}: l \in \mathbb{N}_{0}\right\}$ of $L^{2}(\mathbb{R}) \oplus L^{2}(\mathbb{R})$ is in the range of $d_{\lambda}^{2}+1$, which proves that the range of $\left(\operatorname{id}_{C(\mathbb{T})} \odot d_{\lambda}\right)^{2}+1$ is dense, so $\operatorname{id}_{C(\mathbb{T})} \otimes d_{\lambda}$ is self-adjoint and regular. Moreover, $d_{\lambda}^{2}+1$ is diagonalizable and its eigenvalues $(2 l+1)|\lambda|$ tend to infinity. This shows that $d_{\lambda}^{2}+1$ has compact inverse, and that $\left(\left(\operatorname{id}_{C(\mathbb{T})} \otimes d_{\lambda}\right)^{2}+1\right)^{-1}=\operatorname{id}_{C(\mathbb{T})} \otimes\left(d_{\lambda}^{2}+1\right)^{-1}$ is compact as tensor product of compact operators. Thus, $\operatorname{id}_{C(\mathbb{T})} \otimes d_{\lambda}$ has compact resolvent.

Lemma 4.3. The operator $\operatorname{id}_{C(\mathbb{T})} \otimes d_{\lambda}$ is almost equivariant, i.e. for any $n \in \mathbb{Z}$, the operator $\left(\mathrm{id}_{C(\mathbb{T})} \otimes d_{\lambda}\right)-\mathrm{Ad}_{n}\left(\mathrm{id}_{C(\mathbb{T})} \otimes d_{\lambda}\right)$ on $\operatorname{Dom}\left(\mathrm{id}_{C(\mathbb{T})} \otimes d_{\lambda}\right)$ extends to an adjointable operator. Furthermore, the subalgebra $\left\{f \in C(\mathbb{T}):\left[\operatorname{id}_{C(\mathbb{T})} \otimes d_{\lambda}, f\right] \in \mathcal{L}\left(H_{b}\right)\right\}$ is dense in $C(\mathbb{T})$.

Proof. For $\phi \in C(\mathbb{T}) \odot \mathcal{S}(\mathbb{R}) \subseteq H_{b}{ }^{ \pm}$, we have $n \bullet \frac{\partial \phi}{\partial r}=\frac{\partial}{\partial r}(n \bullet \phi)$, and

$$
\begin{aligned}
n \bullet(\mathrm{M}(-n) \bullet \phi)([x], r) & =(\mathrm{M}(-n) \cdot \phi)([x-n \theta], r-n) \\
& =(r-n) \cdot \phi([x], r) .
\end{aligned}
$$

Hence we have on the dense subspace $(C(\mathbb{T}) \odot \mathcal{S}(\mathbb{R}))^{\oplus 2}$ of $H_{b}$,

$\left(\operatorname{id}_{C(\mathbb{T})} \otimes d_{\lambda}\right)-\operatorname{Ad}_{n}\left(\operatorname{id}_{C(\mathbb{T})} \otimes d_{\lambda}\right)=\left[\begin{array}{cc}0 & \lambda \mathrm{M} \\ \lambda \mathrm{M} & 0\end{array}\right]-\left[\begin{array}{cc}0 & \operatorname{Ad}_{n}(\lambda \mathrm{M}) \\ \operatorname{Ad}_{n}(\lambda \mathrm{M}) & 0\end{array}\right]=\left[\begin{array}{cc}0 & \lambda n \\ \lambda n & 0\end{array}\right]$.

Thus, for any fixed $n \in \mathbb{Z}$, the operator $\left(\operatorname{id}_{C(\mathbb{T})} \otimes d_{\lambda}\right)-\operatorname{Ad}_{n}\left(\operatorname{id}_{C(\mathbb{T})} \otimes d_{\lambda}\right)$ extends to an adjointable operator.

Let $f \in C^{\infty}(\mathbb{T})$. We have $\mathrm{M}(f \bullet \phi)=f \bullet(\mathrm{M} \phi)$, as $f \bullet$ does not change the $\mathbb{R}$-coordinate. Secondly, define

$$
f_{b}([x], r):=f([x+b r]),
$$

so that $f \bullet \phi=f_{b} \cdot \phi$, and

$$
\frac{\partial(f \bullet \phi)}{\partial r}-f \bullet \frac{\partial \phi}{\partial r}=\frac{\partial f_{b}}{\partial r} \cdot \phi .
$$

This is a bounded operator of $\phi$, i.e.

$$
H_{b}{ }^{ \pm} \supseteq C(\mathbb{T}) \odot \mathcal{S}(\mathbb{R}) \ni \phi \longmapsto\left(\lambda \mathrm{M} \pm \frac{\partial}{\partial r}\right)(f \bullet \phi)-f \bullet\left(\lambda \mathrm{M} \pm \frac{\partial}{\partial r}\right)(\phi)= \pm \frac{\partial f_{b}}{\partial r} \cdot \phi
$$

extends to an adjointable operator on $H_{b}^{ \pm}$with adjoint $\phi \mapsto \pm \frac{\partial \overline{f_{b}}}{\partial r} \cdot \phi$. Thus, the dense subalgebra $C^{\infty}(\mathbb{T})$ of $C(\mathbb{T})$ is contained in $\left\{f \in C(\mathbb{T}):\left[\operatorname{id}_{C(\mathbb{T})} \otimes d_{\lambda}, f\right] \in \mathcal{L}\left(H_{b}\right)\right\}$.

This concludes the proof of Theorem 4.1

It follows that $j\left(\left(H_{b}, \operatorname{id}_{C(\mathbb{T})} \otimes d_{\lambda}\right)\right)=:\left(\mathcal{H}_{b}, D_{\lambda}\right)$ is a cycle in $\Psi_{0}\left(A_{\theta}, A_{\theta}\right)$, where $j$ is the descent map on cycles $\Psi_{0}^{\mathbb{Z}}(C(\mathbb{T}), C(\mathbb{T})) \rightarrow \Psi_{0}\left(C(\mathbb{T}) \rtimes_{\theta} \mathbb{Z}, C(\mathbb{T}) \rtimes_{\theta} \mathbb{Z}\right)=$ $\Psi_{0}\left(A_{\theta}, A_{\theta}\right)$. For reference, let us explicitly describe the structure of $\mathcal{H}_{b}$, which can be constructed using descent and the definition of its lift $H_{b}$ on page 263.

Lemma 4.4. The left $\mathfrak{A}$-action on $C_{c}(\mathbb{Z} \times \mathbb{T} \times \mathbb{R}) \subseteq \mathcal{H}_{b}^{ \pm}$is given by

$$
\left(a \cdot \mathcal{H}_{b} \Phi\right)(n,[x], r)=\sum_{m \in \mathbb{Z}} a([x+r b], m) \Phi(n-m,[x-m \theta], r-m) .
$$


and the right action by

$$
\left(\Phi \cdot \mathcal{H}_{b} a\right)(n,[x], r)=\sum_{m \in \mathbb{Z}} \Phi(m,[x], r) a([x-m \theta], n-m) .
$$

Its (pre-) inner product is given by:

$$
\left\langle\Phi_{1} \mid \Phi_{2}\right\rangle_{A_{\theta}}^{\mathcal{H}_{b}}\left([w], l_{2}\right)=\sum_{k_{1}} \int_{\mathbb{R}} \overline{\Phi_{1}}\left(k_{1},\left[w+k_{1} \theta\right], r\right) \Phi_{2}\left(l_{2}+k_{1},\left[w+k_{1} \theta\right], r\right) \mathrm{d} r .
$$

Definition 4.5. The b-twist $\tau_{b}$ is the element of $\mathrm{KK}_{0}\left(A_{\theta}, A_{\theta}\right)$ represented by the descent $\left(\mathcal{H}_{b}, D_{1}\right)$ of the $\mathbb{Z}$-equivariant unbounded cycle $\left(H_{b}, \mathrm{id}_{C(\mathbb{T})} \otimes d_{1}\right)$ for $\mathrm{KK}_{0}^{\mathbb{Z}}(C(\mathbb{T}), C(\mathbb{T}))$.

Remark 4.6. Note that since we have proved that $d_{\lambda}$ defines an elliptic operator for any real $\lambda \neq 0$, any two of the cycles $\left(H_{b}, i_{C(\mathbb{T})} \otimes d_{\lambda}\right)$ with $\lambda$ of the same sign, are homotopic to each other as unbounded Kasparov modules from $C^{\infty}(\mathbb{T})$ to $C(\mathbb{T})$ in the sense of [11, Definition 4.4]. In particular, by Theorem 4.1 of the same preprint, they represent the same class in KK-theory. (Of course, $d_{\lambda}$ is not homotopic to $d_{-\lambda}$, since their nonzero Fredholm indices have opposite signs.)

The duality result we are proving in this article, like all dualities known to the authors, uses Bott Periodicity (specifically in this case, $\mathbb{Z}$-equivariant Bott Periodicity) at some point in the proof. In our case, it is embedded in the proof of the following result.

Theorem 4.7. The twist morphisms $\left\{\tau_{b}\right\}_{b \in \mathbb{Z}} \in \mathrm{KK}_{0}\left(A_{\theta}, A_{\theta}\right)$ form a cyclic group of KK-equivalences under composition. In particular,

$$
\tau_{-b}=\tau_{b}^{-1} \in \mathrm{KK}_{0}\left(A_{\theta}, A_{\theta}\right) .
$$

Recall that Kasparov's bivariant category $\operatorname{RKK}_{*}^{\mathbb{Z}}(\mathbb{R} ; \cdot, \cdot)$ has objects $\mathbb{Z}-\mathrm{C}^{*}$ algebras and morphisms $A \rightarrow B$ are the elements of the abelian group

$$
\mathrm{RKK}_{*}^{\mathbb{Z}}(\mathbb{R} ; A, B),
$$

which is the quotient of the set of cycles $(\mathcal{E}, F)$ for $\mathrm{KK}_{*}^{\mathbb{Z}}\left(C_{0}(\mathbb{R}) \otimes A, C_{0}(\mathbb{R}) \otimes B\right)$ for which the left and right actions of $C_{0}(\mathbb{R})$ on the module $\mathcal{E}$ are equal, by homotopy (with a similar requirement on the homotopy). See [14, 2.19].

Such a cycle can be considered as a family $\left(\mathcal{E}_{t}, F_{t}\right)_{t \in \mathbb{R}}$ of $\mathrm{KK}_{*}(A, B)$-cycles which is essentially equivariant in the sense that, for all $t \in \mathbb{R}$, any integer $l$ maps $\mathcal{E}_{t}$ to $\mathcal{E}_{t+l}$ and

$$
(-l) \circ F_{t+l} \circ l-F_{t}
$$

is a compact operator on $\mathcal{E}_{t}$.

Let

$$
p_{\mathbb{R}}^{*}: \operatorname{KK}_{*}^{\mathbb{Z}}(A, B) \rightarrow \operatorname{RKK}_{*}^{\mathbb{Z}}(\mathbb{R} ; A, B)
$$

be Kasparov's inflation map, which (on cycles) associates to a cycle for $\mathrm{KK}_{*}(A, B)$ the corresponding constant field of cycles over $\mathbb{R}$. The inflation map converts analytic problems into topological problems, as we shall see shortly in connection with our own problems.

The following result follows from the Dirac-dual-Dirac method.

Lemma 4.8 (See [8, Theorem 54]). $p_{\mathbb{R}}^{*}$ is an isomorphism for all $A, B$.

We will be setting $A=B=C(\mathbb{T})$ in the following, and apply the inflation map to the class of the equivariant cycles $\left(H_{b}, \operatorname{id}_{C(\mathbb{T})} \otimes d_{\lambda}\right)$ discussed above. 
Definition 4.9. The topological b-twist $\widehat{\tau}^{b} \in \mathrm{RKK}_{0}^{\mathbb{Z}}(\mathbb{R} ; C(\mathbb{T}), C(\mathbb{T}))$ is the class of the bundle of $*$-homomorphisms

$$
\widehat{\tau}_{t}^{b}: C(\mathbb{T}) \rightarrow C(\mathbb{T}), \quad \widehat{\tau}_{t}^{b}(f)([x]):=f([x+b t]),
$$

The family of automorphisms $\left\{\widehat{\tau}_{t}^{b}\right\}_{t \in \mathbb{R}}$ is equivariant if the action by $\mathbb{Z}$ on $\mathbb{R}$ is by translation and on $C(\mathbb{T})$ is by irrational rotation, since $b$ is an integer.

Since the Kasparov product of two families of automorphisms in $\mathrm{RKK}_{0}^{\mathbb{Z}}$ is simply given by composition, we see that the product of $\widehat{\tau}^{b}$ with $\widehat{\tau}^{b^{\prime}}$ is exactly $\widehat{\tau}^{b+b^{\prime}}$. Clearly, $\widehat{\tau}^{0}$ is the identity, and so we conclude that $b \mapsto \widehat{\tau}^{b}$ is a group homomorphism from $\mathbb{Z}$ to invertibles in $\operatorname{RKK}_{0}^{\mathbb{Z}}(\mathbb{R} ; C(\mathbb{T}), C(\mathbb{T})$ ) (under composition).

Theorem 4.10. Let $\left(H_{b}, \mathrm{id}_{C(\mathbb{T})} \otimes d_{\lambda}\right)$ be the Dirac-Schrödinger cycle for $\mathrm{KK}_{0}^{\mathbb{Z}}(C(\mathbb{T}), C(\mathbb{T}))$ of Theorem 4.1, with $\lambda>0$. Then

$$
p_{\mathbb{R}}^{*}\left(\left[\left(H_{b}, \operatorname{id}_{C(\mathbb{T})} \otimes d_{\lambda}\right)\right]\right)=\widehat{\tau}^{b} \in \operatorname{RKK}_{0}^{\mathbb{Z}}(\mathbb{R} ; C(\mathbb{T}), C(\mathbb{T})) .
$$

Proof. As explained at the beginning of this section, $p_{\mathbb{R}}^{*}\left(\left[\left(H_{b}, \operatorname{id}_{C(\mathbb{T})} \otimes d_{\lambda}\right)\right]\right)$ is represented by the constant bundle of cycles which consists, for each $t \in \mathbb{R}$, of the Dirac-Schrödinger cycle.

First, we will modify the operator

$$
d_{\lambda}=\left[\begin{array}{cc}
0 & d_{\lambda,-} \\
d_{\lambda,+} & 0
\end{array}\right], \quad d_{\lambda, \pm}=\lambda \mathrm{M}_{ \pm} \frac{\partial}{\partial r},
$$

on $L^{2}(\mathbb{R}) \oplus L^{2}(\mathbb{R})$ by changing the implicit reference point $t=0$ in the cycle; we do this to turn our constant family over $\mathbb{R}$, which is essentially $\mathbb{Z}$-equivariant in the sense of Equation (4.6), into a $\mathbb{Z}$-equivariant family. We will then apply an argument of Lück-Rosenberg.

If $U_{t}$ is a left translation unitary with $t \in \mathbb{R}$, then

$$
U_{t} \circ d_{\lambda,+} \circ U_{-t}=\lambda(\mathrm{M}-t)+\frac{\partial}{\partial r}=: d_{\lambda,+}^{t},
$$

and a similar statement holds for $d_{\lambda,-}$ and hence for $d_{\lambda}$. We thus obtain an equivariant family of operators $d_{\lambda}^{t}$ on $L^{2}(\mathbb{R}) \oplus L^{2}(\mathbb{R})$, all unitary conjugates and bounded perturbations of each other since

$$
d_{\lambda}-d_{\lambda}^{t}=d_{\lambda}-U_{t} \circ d_{\lambda} \circ U_{-t}=\left[\begin{array}{cc}
0 & \lambda t \\
\lambda t & 0
\end{array}\right]
$$

in particular, they represent the same class in KK-theory by [11, Proposition 4.7]. We now tensor $d_{\lambda}^{t}$ by the identity on $C(\mathbb{T})$ to obtain a family

$$
\left\{\left(H_{b}, \operatorname{id}_{C(\mathbb{T})} \otimes d_{\lambda}^{t}\right)\right\}_{t}
$$

of cycles for $\mathrm{KK}_{0}^{\mathbb{Z}}(C(\mathbb{T}), C(\mathbb{T}))$, in which only the operator is varying with $t \in \mathbb{R}$ while the modules $H_{b}$ stay constant. This describes a cycle that is a bounded perturbation of the constant cycle which represents $p_{\mathbb{R}}^{*}\left[\left(H_{b}, \mathrm{id}_{C(\mathbb{T})} \otimes d_{\lambda}\right)\right]$. In particular,

$$
p_{\mathbb{R}}^{*}\left(\left[\left(H_{b}, \operatorname{id}_{C(\mathbb{T})} \otimes d_{\lambda}\right)\right]\right)=\left[\left(H_{b}, \operatorname{id}_{C(\mathbb{T})} \otimes d_{\lambda}^{t}\right)_{t \in \mathbb{R}}\right] \in \operatorname{RKK}_{0}^{\mathbb{Z}}(\mathbb{R} ; C(\mathbb{T}), C(\mathbb{T})) .
$$

and our new bundle of cycles is $\mathbb{Z}$-equivariant on the nose, as a bundle.

We next describe a homotopy, which we will describe as a family of homotopies parameterized by $t \in \mathbb{R}$. Fix $t$. 
The following is based on arguments of Lück and Rosenberg in [17]. For $\lambda \epsilon$ $[1,+\infty)$, the spectrum of the operator

$$
d_{\lambda}^{t}:=\left[\begin{array}{cc}
0 & \lambda(\mathrm{M}-t)-\frac{\partial}{\partial r} \\
\lambda(\mathrm{M}-t)+\frac{\partial}{\partial r} & 0
\end{array}\right]
$$

on $L^{2}(\mathbb{R}) \oplus L^{2}(\mathbb{R})$ is given by

$$
\{( \pm \sqrt{2 l+1}) \lambda: l=0,1,2, \ldots\}
$$

and $d_{\lambda}^{t}$ is orthogonally diagonalizable with eigenspaces all of multiplicity 1 . The kernel of $d_{\lambda}^{t}$ is spanned by the unit vector $\psi_{0, \lambda}^{t} \oplus 0$ where

$$
\psi_{0, \lambda}^{t}(r)=\left(\frac{\lambda}{\sqrt{\pi}}\right)^{\frac{1}{2}} \cdot e^{-\frac{\lambda(r-t)^{2}}{2}},
$$

and the Fredholm index of $d_{\lambda}^{t}$ is 1 .

For each $\lambda$, let $\operatorname{pr}_{\lambda}^{t}$ be projection to the kernel of $d_{\lambda}$. Since the minimal nonzero eigenvalue of $d_{\lambda}^{t}$ has a distance $\sqrt{2 \lambda}$ to the origin, we obtain Part (1) of the following

Lemma 4.11. With $d_{\lambda}^{t}$ as above and $f\left(d_{\lambda}^{t}\right) \in \mathcal{L}\left(L^{2}(\mathbb{R})^{\oplus 2}\right)$ the operator obtained from $f \in C_{0}(\mathbb{R})$ by functional calculus, we have

(1) $\lim _{\lambda \rightarrow+\infty}\left\|f\left(d_{\lambda}^{t}\right)-f(0) \cdot \operatorname{pr}_{\lambda}^{t}\right\|=0$.

(2) If $\chi \in C_{b}(\mathbb{R})$ is a normalizing function, and $\epsilon^{t}$ is the (Borel measurable) sign function on $\mathbb{R}$ given by

$$
\epsilon^{t}(r):=\frac{r-t}{|r-t|},
$$

acting as a multiplication operator on $L^{2}(\mathbb{R})$, then

$$
F_{\lambda, t}:=\chi\left(d_{\lambda}^{t}\right) \rightarrow\left[\begin{array}{cc}
0 & \epsilon^{t} \\
\epsilon^{t} & 0
\end{array}\right] \text { for } \lambda \rightarrow+\infty
$$

in the strong operator topology.

(3) If $f$ is a smooth, periodic function on $\mathbb{R}$, then

$$
\lim _{\lambda \rightarrow+\infty}\left\|\left[F_{\lambda, t}, f\right]\right\|=0 .
$$

The proof of (2) is carried out in [17, p. 582-583], and of (3) in [17, p. 584-586]. Define a family $\left\{W_{\lambda, t}\right\}_{\lambda}=\left\{W_{\lambda, t}^{+} \oplus W_{\lambda, t}^{-}\right\}_{\lambda \in[1,+\infty}$ of Hilbert spaces by setting $W_{\lambda, t}^{-}:=L^{2}(\mathbb{R})$ for all $\lambda \in[1,+\infty]$, and

$$
W_{\lambda, t}^{+}= \begin{cases}L^{2}(\mathbb{R}) & \text { if } 1 \leq \lambda<\infty, \\ L^{2}(\mathbb{R}) \oplus \mathbb{C} & \text { if } \lambda=\infty\end{cases}
$$

We let $\delta_{0}^{t}=(0,1) \in W_{\infty, t}^{+}=L^{2}(\mathbb{R}) \oplus \mathbb{C}$.

To endow this field with a structure of a continuous field, we only need to be concerned about the point $\infty$ : We declare a section $\xi^{t}$ of the field $\left\{W_{\lambda, t}^{+}\right\}_{\lambda \in[1,+\infty]}$ with value $f+z \delta_{0}^{t}$ at $\lambda=+\infty, f \in L^{2}(\mathbb{R})$ and $z \in \mathbb{C}$, to be continuous at infinity if

$$
\left\|\xi^{t}(\lambda)-\left(f+z \psi_{0, \lambda}^{t}\right)\right\|_{L^{2}(\mathbb{R})} \rightarrow 0 \quad \text { as } \lambda \rightarrow+\infty,
$$

where $\psi_{0, \lambda}^{t} \in L^{2}(\mathbb{R})$ is the normalized 0-eigenvector of $d_{\lambda}^{t}$ as defined in Equation (4.8).

We now describe a continuous family of self-adjoint, grading-reversing operators

$$
F_{\lambda, t}: W_{\lambda, t} \rightarrow W_{\lambda, t}
$$


for $\lambda \in[1,+\infty]$. For finite $\lambda$, set

$$
F_{\lambda, t}:=\chi\left(d_{\lambda}^{t}\right), \quad \text { where } d_{\lambda}^{t}=\left[\begin{array}{cc}
0 & \lambda(\mathrm{M}-t)-\frac{\partial}{\partial r} \\
\lambda(\mathrm{M}-t)+\frac{\partial}{\partial r} & 0
\end{array}\right] .
$$

This odd, self-adjoint operator has the form

$$
F_{\lambda, t}=\left[\begin{array}{cc}
0 & G_{\lambda, t}^{*} \\
G_{\lambda, t} & 0
\end{array}\right]
$$

for suitable $G_{\lambda, t}$.

At infinity, we have $W_{\infty, t}=\left(L^{2}(\mathbb{R}) \oplus \mathbb{C}\right) \oplus L^{2}(\mathbb{R})$ with the first summand $L^{2}(\mathbb{R}) \oplus \mathbb{C}$ graded even and the second summand $L^{2}(\mathbb{R})$ graded odd. We let

$$
G_{\infty, t}: L^{2}(\mathbb{R}) \oplus \mathbb{C} \rightarrow L^{2}(\mathbb{R})
$$

be multiplication by the sign function $\epsilon^{t}$ on the summand $L^{2}(\mathbb{R})$, and zero on the $\mathbb{C}$-summand. Thus, the operator $G_{\infty, t}^{*}: L^{2}(\mathbb{R}) \rightarrow L^{2}(\mathbb{R}) \oplus \mathbb{C}$ is multiplication by $\epsilon^{t}$ on $L^{2}(\mathbb{R})$, followed by the inclusion into $L^{2}(\mathbb{R}) \oplus \mathbb{C}$ by zero in the second summand. The operator $F_{\infty, t}$ is the odd, self-adjoint operator on $W_{\infty, t}$ given by the matrix

$$
F_{\infty, t}:=\left[\begin{array}{cc}
0 & G_{\infty, t}^{*} \\
G_{\infty, t} & 0
\end{array}\right]
$$

This is the correct choice in order to make $\left(F_{\lambda, t}\right)_{\lambda}$ a continuous family, i.e. an adjointable operator on the module of sections, because of (4.9) in Lemma 4.11] Note that the operator $L^{2}(\mathbb{R}) \rightarrow L^{2}(\mathbb{R})$ of multiplication by $\epsilon^{t}$ has no kernel. Since, however, $G_{\infty, t}$ kills the second summand $\mathbb{C}$ of $L^{2}(\mathbb{R}) \oplus \mathbb{C}$, the operator $G_{\infty, t}$ has a 1-dimensional kernel. The cokernel of $G_{\infty, t}$ is clearly trivial, and therefore $G_{\infty, t}$ (and $F_{\infty, t}$ ) also has index 1.

The family of operators $\left\{F_{\lambda, t}\right\}_{\lambda \in[1,+\infty}$ induces an odd, self-adjoint operator $F_{t}$ on the sections $\mathcal{E}_{t}$ of the field $\left\{W_{\lambda, t}\right\}_{\lambda \in[1,+\infty]}$. In other words, we have constructed a $\mathbb{Z} / 2$-graded Hilbert $C([1,+\infty])$-module and an odd, self-adjoint operator $F_{t}$ on $\mathcal{E}_{t}$. Further, $1-F_{t}^{2}$ is compact: for finite $\lambda$,

$$
1-F_{\lambda, t}^{2}=\left(1-\chi^{2}\right)\left(d_{\lambda}^{t}\right)
$$

is compact by Lemma 4.11 By the same lemma,

$$
\left\|\left(1-F_{\lambda, t}^{2}\right)-\left(1-\chi^{2}\right)(0) \cdot \operatorname{pr}_{\lambda, t}\right\|=\left\|1-F_{\lambda, t}^{2}-\operatorname{pr}_{\lambda, t}\right\| \rightarrow 0 \text { for } \lambda \rightarrow \infty .
$$

As $\operatorname{pr}_{\lambda}^{t}=\left|\psi_{0, \lambda}^{t}\right\rangle\left\langle\psi_{0, \lambda}^{t}\right|$ and $1-F_{\infty, t}^{2}=(0 \oplus 1) \oplus 0=\left|\delta_{0}^{t}\right\rangle\left\langle\delta_{0}^{t}\right|$ on $\left(L^{2}(\mathbb{R}) \oplus \mathbb{C}\right) \oplus L^{2}(\mathbb{R})$, we see that $1-F_{\lambda, t}^{2}$ is asymptotic to $|\xi\rangle\langle\xi|$, the rank-one operator corresponding to the continuous section given by $\xi_{\lambda}:=\psi_{0, \lambda}^{t}$ for $\lambda<\infty$ and $\xi(\infty)=\delta_{0}^{t}$.

The definitions above supply a homotopy of $\mathrm{KK}_{0}(\mathbb{C}, \mathbb{C})$-cycles between the bounded transform $\left(W_{\lambda, t}, F_{\lambda, t}\right)$ of $\left(W_{\lambda, t}, d_{\lambda}^{t}\right)=\left(L^{2}(\mathbb{R}) \oplus L^{2}(\mathbb{R}), d_{\lambda}^{t}\right)$ for any finite $\lambda$ and any $t \in \mathbb{R}$, on the one hand, and the sum of the cycle $(\mathbb{C} \oplus 0,0)$ with the degenerate cycle

$$
\left(L^{2}(\mathbb{R}) \oplus L^{2}(\mathbb{R}),\left[\begin{array}{cc}
0 & \epsilon^{t} \\
\epsilon^{t} & 0
\end{array}\right]\right)
$$


on the other hand. Here, both $\mathbb{C} \oplus 0$ and $L^{2}(\mathbb{R}) \oplus L^{2}(\mathbb{R})$ are $\mathbb{Z} / 2$-graded with their respective first summand even and second odd, and $\epsilon^{t}$ is the sign function as before.

Further, the homotopy is equivariant for $\mathbb{Z}$ if one allows the real parameter $t \in \mathbb{R}$ to change with the integer action: translation by $n \in \mathbb{Z}$ conjugates $d_{\lambda}^{t}$ to $d_{\lambda}^{t+n}$. This means that the construction can be carried out in $\operatorname{RKK}^{\mathbb{Z}}(\mathbb{R} ; \cdot, \cdot)$, as we now show.

Set

$$
\mathcal{E}_{\lambda, t}:=C(\mathbb{T}) \otimes W_{\lambda, t} \quad \text { and } \quad \mathcal{F}_{\lambda, t}:=\mathrm{id}_{C(\mathbb{T})} \otimes F_{\lambda, t},
$$

endowed with its standard right Hilbert $C(\mathbb{T})$-module structure, and carrying the $\mathbb{Z} / 2$-grading inherited from the gradings on $W_{\lambda, t}$. On $\mathcal{E}_{\lambda, t}$ and for $f \in C(\mathbb{T})$ considered a periodic function on $\mathbb{R}$, we let

$$
\nu_{\lambda, t}(f) \in \mathcal{L}\left(\mathcal{E}_{\lambda, t}\right)
$$

be the operator defined as follows. Set

$$
f_{b}([x], r)=f([x+b r]),
$$

where $b$ is the integer which was fixed in the beginning. For finite $\lambda$, we let $\nu_{\lambda, t}^{ \pm}(f)$ act on $\mathcal{E}_{\lambda, t}^{ \pm}=C(\mathbb{T}) \otimes L^{2}(\mathbb{R})$ by multiplication by the function $f_{b}$ on $\mathbb{T} \times \mathbb{R}$. For $\lambda=\infty$, we let $\nu_{\infty, t}^{+}(f)$ act on $\mathcal{E}_{\infty, t}^{+}=C(\mathbb{T}) \otimes\left(L^{2}(\mathbb{R}) \oplus \mathbb{C}\right)=\left(C(\mathbb{T}) \otimes L^{2}(\mathbb{R})\right) \oplus C(\mathbb{T})$ by multiplication by $f_{b}$ on the first factor $C(\mathbb{T}) \otimes L^{2}(\mathbb{R})$, and on the second factor $C(\mathbb{T})$ by multiplication by the function $f_{b}^{t} \in C(\mathbb{T})$, where

$$
f_{b}^{t}([x]):=f([x+b t]) .
$$

For $t \in \mathbb{R}$ and $\lambda \in(0, \infty]$, let

$$
Y_{\lambda, t}:=\left(\nu_{\lambda, t}, \mathcal{E}_{\lambda, t}, \mathcal{F}_{\lambda, t}\right) \quad \text { and } \quad Y_{\lambda}:=\left\{Y_{\lambda, t}\right\}_{t \in \mathbb{R}} .
$$

These RKK-cycles are $\mathbb{Z}$-equivariant, and $\left(\lambda \mapsto Y_{\lambda}\right)$ is a homotopy of RKK $\mathbb{Z}^{\mathbb{Z}}$-cycles. For any $\lambda \in(0, \infty)$, Equation (4.7) yields that $Y_{\lambda}$ is a compact perturbation of the constant family $\left\{Y_{\lambda, 0}\right\}_{t \in \mathbb{R}}$, because they arise as the bounded transform of $\left\{\left(L^{2}(\mathbb{R}) \oplus\right.\right.$ $\left.\left.L^{2}(\mathbb{R}), d_{\lambda}^{t}\right)\right\}_{t \in \mathbb{R}}$ resp. $\operatorname{pr}_{\mathbb{R}}^{*}\left(L^{2}(\mathbb{R}) \oplus L^{2}(\mathbb{R}), d_{\lambda}\right)$ after fibrewise tensoring with the rightHilbert $C(\mathbb{T})$-bimodule $(\nu, C(\mathbb{T}))$. Thus, $Y_{\lambda}$ and $\left\{Y_{\lambda, 0}\right\}_{t \in \mathbb{R}}$ determine the same class in $\mathrm{RKK}^{\mathbb{Z}}$. By definition of the inflation map, $\operatorname{pr}_{\mathbb{R}}^{*}\left(\left(H_{b}, \mathrm{id}_{C(\mathbb{T})} \otimes d_{\lambda}\right)\right)=\left\{Y_{\lambda, 0}\right\}_{t \in \mathbb{R}}$ for any finite $\lambda$, so we have shown that $\operatorname{pr}_{\mathbb{R}}^{*}\left(\left(H_{b}, \operatorname{id}_{C(\mathbb{T})} \otimes d_{\lambda}\right)\right)$ and $Y_{\lambda}$ determine the same class.

On the other hand, at $\lambda=\infty$, we have that $Y_{\infty}$ is the sum of the topological $b$-twist $\widehat{\tau}^{b}=\left\{\widehat{\tau}_{t}^{b}\right\}_{t \in \mathbb{R}}$, see Definition 4.9, and the degenerate $\left(t \mapsto\left(H_{b},\left[\begin{array}{cc}0 & \epsilon^{t} \\ \epsilon^{t} & 0\end{array}\right]\right)\right)$. In particular, $\widehat{\tau}^{b}$ also determines the same class as $Y_{\lambda}$ in $\mathrm{RKK}^{\mathbb{Z}}$. This concludes our proof of Theorem 4.10

Proof of Theorem 4.7, Since $\mathrm{pr}_{\mathbb{R}}^{*}$ is an isomorphism, it follows from Theorem 4.10 that $b \mapsto\left[\left(H_{b}, \mathrm{id}_{C(\mathbb{T})} \otimes d_{\lambda}\right)\right]$ is a group homomorphism from $\mathbb{Z}$ to $\operatorname{KK}_{0}^{\mathbb{Z}}(C(\mathbb{T}), C(\mathbb{T}))$. Using descent, the map

$$
b \mapsto j\left[\left(H_{b}, \operatorname{id}_{C(\mathbb{T})} \otimes d_{\lambda}\right)\right]=\left[\left(\mathcal{H}_{b}, D_{\lambda}\right)\right]=\tau_{b}
$$

is a group homomorphism from $\mathbb{Z}$ to $\mathrm{KK}_{0}\left(A_{\theta}, A_{\theta}\right)$, as claimed. 


\section{CONnes' DUALity AND TRANSVERsals}

Let $\Delta_{\theta} \in \mathrm{KK}_{0}\left(A_{\theta} \otimes A_{\theta}, \mathbb{C}\right)$ be Connes' class of Definition 2.3, The main technical result of this paper is the following.

Theorem 5.1. Let $g=\left[\begin{array}{ll}1 & b \\ 0 & 1\end{array}\right]$ for $b \neq 0$ and $\mathcal{L}_{b}:=\mathcal{L}_{g}$. Then

$$
\left(1_{A_{\theta}} \otimes\left[\mathcal{L}_{b}\right]\right) \otimes_{A_{\theta}^{\otimes 3}}\left(\Delta_{\theta} \otimes 1_{A_{\theta}}\right)=\left[\left(\mathcal{H}_{b}, \frac{1}{b} D_{2 \pi b}\right)\right] \in \mathrm{KK}_{0}\left(A_{\theta}, A_{\theta}\right) .
$$

In particular, if $b>0$, then this class coincides with $\tau_{b} \in \mathrm{KK}_{0}\left(A_{\theta}, A_{\theta}\right)$, the b-twist (Definition 4.5).

We proceed to the proof of Theorem 5.1

5.1. Computation of the module in the zig-zag product. Our goal is to compute $\left(1_{A_{\theta}} \otimes\left[\mathcal{L}_{g}\right]\right) \otimes_{A_{\theta}^{\otimes 3}}\left(\Delta_{\theta} \otimes 1_{A_{\theta}}\right) \in \mathrm{KK}_{0}\left(A_{\theta}, A_{\theta}\right)$ for $g$ upper-triangular, and prove that it equals the class of the $b$-twist of Theorem 4.1

In fact, some of the calculations we will do for arbitrary $g$, since it involves little additional effort and leads to the following observation: only for upper-triangular $g$, the Hilbert $A_{\theta}$-bimodule involved in the Kasparov product of the left hand side of (5.1) is of the kind one gets from applying descent to an equivariant module (such as the one appearing in our cycle for the $b$-twist).

As the module $\mathcal{L}_{g}$ and the $\mathrm{C}^{*}$-algebra $A_{\theta}$ are ungraded, the module underlying this class is comprised of two copies of

$$
\left(A_{\theta} \otimes \mathcal{L}_{g}\right) \otimes_{A_{\theta}^{\otimes 3}}\left(L^{2} \otimes A_{\theta}\right),
$$

where $L^{2}=L^{2}(\mathbb{T}) \otimes \ell^{2}(\mathbb{Z})$ as before (see Lemma 2.2). We initially focus on describing this bimodule. Observe first that one is reduced to computing $\mathcal{L}_{g} \otimes_{A_{\theta}} L^{2}$, where the balancing is over $A_{\theta} \otimes 1$ acting on the right of $\mathcal{L}_{g}$, and $A_{\theta}$ acting on the left of $L^{2}$ via $\omega_{2} \rtimes v$. This is because the maps

$$
\left(A_{\theta} \otimes \mathcal{L}_{g}\right) \otimes_{A_{\theta}^{\otimes 3}}\left(L^{2} \otimes A_{\theta}\right) \longleftrightarrow \mathcal{L}_{g} \otimes_{A_{\theta}} L^{2}
$$

defined on elementary tensors by

$$
\begin{aligned}
& (a \otimes \Phi) \otimes(f \otimes b) \longmapsto \Phi \cdot \mathcal{L}_{g}(1 \otimes b) \otimes\left(\omega_{1} \rtimes u\right)(a)(f) \\
& (1 \otimes \Phi) \otimes(f \otimes 1) \longleftrightarrow \Phi \otimes f
\end{aligned}
$$

are inverse to one another and therefore equip the right-hand side with the structure of a right-Hilbert $A_{\theta}$-bimodule as follows:

$$
\begin{array}{ll}
A_{\theta} \curvearrowright\left(\mathcal{L}_{g} \otimes_{A_{\theta}} L^{2}\right): & \xi(\Phi \otimes f):=\Phi \otimes\left(\omega_{1} \rtimes u\right)(\xi)(f), \\
\left(\mathcal{L}_{g} \otimes_{A_{\theta}} L^{2}\right) \curvearrowleft A_{\theta}: & (\Phi \otimes f) \xi:=\Phi \cdot \mathcal{L}_{g}(1 \otimes \xi) \otimes f .
\end{array}
$$

Moreover, $\mathcal{L}_{g} \otimes_{A_{\theta}} L^{2}$ has $A_{\theta} \otimes A_{\theta}$-valued inner product given on elementary tensors by

$\left\langle\Phi \otimes f_{1} \mid \Psi \otimes f_{2}\right\rangle=\left\langle\left\langle(1 \otimes \Phi) \otimes\left(f_{1} \otimes 1\right) \mid(1 \otimes \Psi) \otimes\left(f_{2} \otimes 1\right)\right\rangle\right\rangle^{\left(1_{A_{\theta}} \otimes \mathcal{L}_{g}\right) \otimes_{A_{\theta} \otimes 3}\left(L^{2} \otimes 1_{A_{\theta}}\right)}$

$$
=\left\langle f_{1} \otimes 1 \mid\left(\langle 1 \otimes \Phi \mid 1 \otimes \Psi\rangle_{A_{\theta}^{\otimes 3}}^{A_{\theta} \otimes \mathcal{L}_{g}}\right) \cdot\left(f_{2} \otimes 1\right)\right\rangle^{L^{2} \otimes A_{\theta}},
$$

where $\cdot$ denotes, for the moment, the left-action of $A_{\theta}^{\otimes 3}$ on $L^{2} \otimes A_{\theta}$. 
Lemma 5.2. The maps

$$
\begin{aligned}
C_{c}\left(\mathcal{Z}_{g}\right) & \longrightarrow C_{c}\left(\mathcal{Z}_{g}\right) \odot_{\mathfrak{A}} \mathfrak{A} \subseteq \mathcal{L}_{g} \otimes_{A_{\theta}} L^{2} \\
\Phi & \longmapsto \Phi \otimes\left(z^{0} \otimes \varepsilon_{0}\right) \\
\Phi \cdot \mathcal{L}_{g}\left(V^{l} U^{-k} \otimes 1\right) & \longleftrightarrow \Phi \otimes\left(z^{l} \otimes \varepsilon_{k}\right)
\end{aligned}
$$

are mutually inverse. In particular with the help of Formula (5.2), a copy of the space $C_{c}\left(\mathcal{Z}_{g}\right)$ is sitting densely inside of $\left(A_{\theta} \otimes \mathcal{L}_{g}\right) \otimes_{A_{\theta}^{\otimes 3}}\left(L^{2} \otimes A_{\theta}\right)$.

In the above lemma, we write $\mathfrak{A}$ for two things: on the one hand, it denotes the dense subspace of $L^{2}$ consisting of elements $\sum_{n, m} a_{n, m} z^{n} \otimes \varepsilon_{m}$. On the other hand, it denotes the subalgebra of $A_{\theta}$ consisting of elements $\sum_{n, m} a_{n, m} V^{n} U^{m}$. In both of these cases, $\left(a_{n, m}\right)_{n, m}$ is assumed to be of Schwartz decay. Recall also that $\odot$ denotes the algebraic tensor product before completion.

Proof. On the right-hand side, the balancing gives us the following equality for $\Phi \in C_{c}\left(\mathcal{Z}_{g}\right), f \in \mathfrak{A} \subseteq L^{2}$, and any acting element $\xi \in \mathfrak{A} \subseteq A_{\theta}$ :

$$
\Phi \otimes\left(\omega_{2} \rtimes v\right)(\xi)(f)=\Phi \cdot \mathcal{L}_{g}(\xi \otimes 1) \otimes f .
$$

For $\xi=V^{l_{1}} U^{k_{1}}$ and $f=z^{l_{2}} \otimes \varepsilon_{k_{2}}$, we have

$$
\left(\omega_{2} \rtimes v\right)(\xi)(f)=\lambda^{-k_{1} l_{2}} z^{l_{2}+l_{1}} \otimes \varepsilon_{k_{2}-k_{1}},
$$

where $\lambda:=\mathrm{e}^{2 \pi i \theta}$. So we have for any choice of $l_{1}, k_{1} \in \mathbb{Z}$ :

$$
\Phi \otimes\left(\lambda^{-k_{1} l_{2}} \cdot z^{l_{2}+l_{1}} \otimes \varepsilon_{k_{2}-k_{1}}\right)=\left(\Phi \cdot \mathcal{L}_{g}\left(V^{l_{1}} U^{k_{1}} \otimes 1\right)\right) \otimes\left(z^{l_{2}} \otimes \varepsilon_{k_{2}}\right) .
$$

The case $k_{2}:=0, l_{2}:=0$ and $k_{1}$ replaced by $-k_{1}$ yields:

$$
\Phi \otimes\left(z^{l_{1}} \otimes \varepsilon_{k_{1}}\right)=\left(\Phi \cdot \mathcal{L}_{g}\left(V^{l_{1}} U^{-k_{1}} \otimes 1\right)\right) \otimes\left(z^{0} \otimes \varepsilon_{0}\right) .
$$

It is now easy to see that the two maps are mutually inverse maps, as claimed.

Formula (5.5) equips the left-hand side with the structure of an $\mathfrak{A}-\mathfrak{A}$-rightpre-Hilbert module. We let $\mathcal{N}_{g}^{0}$ be its completion and $\mathcal{N}_{g}:=\mathcal{N}_{g}^{0} \oplus \mathcal{N}_{g}^{0}$ with the standard even grading. By construction, $\mathcal{N}_{g}$ is (isomorphic to) the $A_{\theta}-A_{\theta}$-rightHilbert module underlying $\left(1_{A_{\theta}} \otimes\left[\mathcal{L}_{g}\right]\right) \otimes_{A_{\theta}^{\otimes 3}}\left(\Delta_{\theta} \otimes 1_{A_{\theta}}\right)$. We will now study this $\mathfrak{A}-\mathfrak{A}$-right-pre-Hilbert module in terms of the bimodule structure of $\mathcal{L}_{g}$.

Lemma 5.3 (The Hilbert bimodule structure of $\mathcal{N}_{g}^{0}$ ). The bimodule structure on $\mathcal{N}_{g}^{0}$ is given on its dense subspace $C_{c}\left(\mathcal{Z}_{g}\right)$ by

$$
\begin{aligned}
& \mathfrak{A} \curvearrowright C_{c}\left(\mathcal{Z}_{g}\right): \quad\left(V^{l_{1}} U^{k_{1}} \square \Phi\right)=\lambda^{l_{1} k_{1}} \Phi \cdot \mathcal{L}_{g}\left(V^{l_{1}} U^{-k_{1}} \otimes 1\right), \\
& C_{c}\left(\mathcal{Z}_{g}\right) \curvearrowleft \mathfrak{A}: \quad\left(\Phi \square V^{l_{2}} U^{k_{2}}\right)=\Phi \cdot \mathcal{L}_{g}\left(1 \otimes V^{l_{2}} U^{k_{2}}\right) .
\end{aligned}
$$

For $\Psi$ another compactly supported function on $\mathcal{Z}_{g}$, the (pre-)inner product $\langle\Phi \mid \Psi\rangle^{\mathcal{N}_{g}^{0}}$ with value in $C_{c}\left(\mathcal{A}_{\theta}\right) \subseteq A_{\theta}$ is given by

$$
\langle\Phi \mid \Psi\rangle^{\mathcal{N}_{g}^{0}}([x], k)=\int_{\mathbb{T}}\langle\Phi \mid \Psi\rangle^{\mathcal{L}_{g}}([y], 0,[x], k) \mathrm{d} y,
$$

where $\langle\Phi \mid \Psi\rangle^{\mathcal{L}_{g}}$ takes values in $C_{c}(\mathcal{A})=C_{c}\left(\mathcal{A}_{\theta} \times \mathcal{A}_{\theta}\right)$. 
Proof. An element $\Phi \in C_{c}\left(\mathcal{Z}_{g}\right)$ corresponds to $\Phi \otimes\left(z^{0} \otimes \varepsilon_{0}\right)$ in $\mathcal{L}_{g} \otimes_{A_{\theta}} L^{2}$, see Formula (5.5). By Formula (5.3), the left action on $\mathcal{L}_{g} \otimes_{A_{\theta}} L^{2}$ is given by

$$
V^{l_{1}} U^{k_{1}} \cdot\left(\Phi \otimes\left(z^{0} \otimes \varepsilon_{0}\right)\right)=\Phi \otimes\left(\omega_{1} \rtimes u\right)\left(V^{l_{1}} U^{k_{1}}\right)\left(z^{0} \otimes \varepsilon_{0}\right) .
$$

We compute

$$
\left(\omega_{1} \rtimes u\right)\left(V^{l_{1}} U^{k_{1}}\right)\left(z^{0} \otimes \varepsilon_{0}\right)=\lambda^{l_{1} k_{1}} z^{l_{1}} \otimes \varepsilon_{k_{1}},
$$

so that

$$
V^{l_{1}} U^{k_{1}} \cdot\left(\Phi \otimes\left(z^{0} \otimes \varepsilon_{0}\right)\right)=\lambda^{l_{1} k_{1}} \Phi \otimes\left(z^{l_{1}} \otimes \varepsilon_{k_{1}}\right) .
$$

Similarly, the right action on $\mathcal{L}_{g} \otimes_{A_{\theta}} L^{2}$ is given by

$$
\left(\Phi \otimes\left(z^{0} \otimes \varepsilon_{0}\right)\right) \cdot V^{l_{2}} U^{k_{2}}=\Phi \cdot \mathcal{L}_{g}\left(1 \otimes V^{l_{2}} U^{k_{2}}\right) \otimes\left(z^{0} \otimes \varepsilon_{0}\right) .
$$

The claim about the bimodule structure now follows from Formula (5.5).

Next, we turn to the inner product. Because of Equation (5.4) and Formula (5.5), the pre-inner product on $\mathcal{N}_{g}^{0}$ is given by

$$
\langle\Phi \mid \Psi\rangle^{\mathcal{N}_{g}^{0}}=\left\langle z^{0} \otimes \varepsilon_{0} \otimes 1 \mid\left(\langle 1 \otimes \Phi \mid 1 \otimes \Psi\rangle_{A_{\theta}^{\otimes 3}}^{A_{\theta} \otimes \mathcal{L}_{g}}\right) \cdot\left(z^{0} \otimes \varepsilon_{0} \otimes 1\right)\right\rangle^{L^{2} \otimes A_{\theta}},
$$

where $\cdot$ is, as before, the left-action of $A_{\theta}^{\otimes 3}$ on $L^{2} \otimes A_{\theta}$. Since

$$
\langle 1 \otimes \Phi \mid 1 \otimes \Psi\rangle_{A_{\theta}^{\otimes 3}}^{A_{\theta} \otimes \mathcal{L}_{g}}=1 \otimes\langle\Phi \mid \Psi\rangle_{A_{\theta} \otimes A_{\theta}}^{\mathcal{L}_{g}},
$$

let us study Equation (5.7) for $\langle 1 \otimes \Phi \mid 1 \otimes \Psi\rangle$ replaced by an elementary tensor $1 \otimes a \otimes b:$

$$
\left\langle z^{0} \otimes \varepsilon_{0} \otimes 1 \mid(1 \otimes a \otimes b) \cdot\left(z^{0} \otimes \varepsilon_{0} \otimes 1\right)\right\rangle^{L^{2} \otimes A_{\theta}}=\left\langle z^{0} \otimes \varepsilon_{0} \mid \omega_{2} \rtimes v(a)\left(z^{0} \otimes \varepsilon_{0}\right)\right\rangle^{L^{2}} \cdot b .
$$

For $a=\sum_{n, m} a_{n, m} V^{n} U^{m}$, we have

$$
\omega_{2} \rtimes v(a)\left(z^{0} \otimes \varepsilon_{0}\right)=\sum_{n, m} a_{n, m} z^{n} \otimes \varepsilon_{-m},
$$

so that

$$
\left\langle z^{0} \otimes \varepsilon_{0} \mid \omega_{2} \rtimes v(a)\left(z^{0} \otimes \varepsilon_{0}\right)\right\rangle^{L^{2}}=a_{0,0} .
$$

Thus, for any $([x], k) \in \mathbb{T} \times \mathbb{Z}$ :

$$
\begin{aligned}
\left\langle z^{0} \otimes \varepsilon_{0} \otimes 1 \mid(1 \otimes a \otimes b) \cdot\left(z^{0} \otimes \varepsilon_{0} \otimes 1\right)\right\rangle_{A_{\theta}}^{L^{2} \otimes A_{\theta}}([x], k) & =a_{0,0} \cdot b([x], k) \\
& =\int_{\mathbb{T}}(a \otimes b)([y], 0,[x], k) \mathrm{d} y .
\end{aligned}
$$

We bootstrap from the elementary tensor $a \otimes b$ with $a \in \mathfrak{A}$ to a more general element $\zeta \in C_{c}(\mathbb{T} \times \mathbb{Z} \times \mathbb{T} \times \mathbb{Z})$ with the result

$$
\left\langle z^{0} \otimes \varepsilon_{0} \otimes 1 \mid(1 \otimes \zeta) \cdot\left(z^{0} \otimes \varepsilon_{0} \otimes 1\right)\right\rangle^{L^{2} \otimes A_{\theta}}([x], k)=\int_{\mathbb{T}} \zeta([y], 0,[x], k) \mathrm{d} y
$$

and so in particular

$$
\begin{aligned}
\langle\Phi \mid \Psi\rangle^{\mathcal{N}_{g}^{0}}([x], k) & =\left\langle z^{0} \otimes \varepsilon_{0} \otimes 1 \mid\left(1 \otimes\langle\Phi \mid \Psi\rangle^{\mathcal{L}_{g}}\right) \cdot\left(z^{0} \otimes \varepsilon_{0} \otimes 1\right)\right\rangle^{L^{2} \otimes A_{\theta}} \\
& =\int_{\mathbb{T}}\langle\Phi \mid \Psi\rangle^{\mathcal{L}_{g}}([y], 0,[x], k) \mathrm{d} y,
\end{aligned}
$$

where the last equation follows from Formula (5.7). 
Our goal is to show that for $g$ upper-triangular, the module $\mathcal{N}_{g}$ underlying the cup-cap product $\left(1_{A_{\theta}} \otimes\left[\mathcal{L}_{g}\right]\right) \otimes_{A_{\theta}^{\otimes 3}}\left(\Delta_{\theta} \otimes 1_{A_{\theta}}\right)$ is obtained by applying the descent map to an equivariant module, and to identify this module. Such 'descended' modules are completions of $C_{c}(\mathbb{Z}, N)$ for some right-Hilbert $C(\mathbb{T})-C(\mathbb{T})$-bimodule $N$ equipped with a $\mathbb{Z}$-action. As already mentioned, $\mathcal{N}_{g}^{0}$ is a completion of continuous compactly supported functions on the space $\mathcal{Z}_{g}$, which for $g=\left[\begin{array}{ll}a & b \\ c & d\end{array}\right]$ is given by

$$
\mathcal{Z}_{\left[\begin{array}{ll}
a & b \\
c & d
\end{array}\right]}=\left\{\left(r_{1}, r_{2},\left[\begin{array}{c}
v \\
w
\end{array}\right]\right) \in \mathbb{R}^{2} \times \mathbb{T}^{2} \mid\left[\begin{array}{l}
a\left(v+r_{1} \theta\right)+b r_{1} \\
c\left(v+r_{1} \theta\right)+d r_{1}
\end{array}\right]=\left[\begin{array}{c}
w+r_{2} \theta \\
r_{2}
\end{array}\right]\right\}
$$

see Lemma 3.8 We therefore need to restrict to those $g$ which make $\mathcal{Z}_{g}$ contain a copy of $\mathbb{Z}$. From the above description, we see that this happens exactly when $g$ is upper triangular; then the elements of $\mathcal{Z}_{g}$ have the restriction $\left[d r_{1}\right]=\left[r_{2}\right]$, i.e. $r_{2}=d r_{1}+k$ for some $k \in \mathbb{Z}$.

Since $g$ was assumed to be in $\mathrm{SL}_{2}(\mathbb{Z}), c=0$ implies $d=a$, and $\mu(g) \neq 0$ (Equation (3.6)) becomes $b \neq 0$. We get

$$
\begin{aligned}
& \mathcal{Z}_{\left[\begin{array}{ll}
a & b \\
0 & a
\end{array}\right]} \cong \mathbb{Z} \times \mathbb{T} \times \mathbb{R} \\
&\left(r, r_{2},\left[\begin{array}{l}
v \\
w
\end{array}\right]\right) \mapsto\left(r_{2}-a r,[v], r\right) .
\end{aligned}
$$

The below proposition gives the formulas that $\mathcal{Z}_{g}$ inherits from $\mathcal{Y}_{g} \star_{\mathcal{B}} \mathcal{X}$ via the identification from Equation (5.8). It also makes use of Lemma 3.7 which gave a nicer description of the left $\mathcal{F}_{g}$-action on $\mathcal{Y}_{g}$, and of Lemma 3.3 which gave a nicer description of the right $\mathcal{A}$-action on $\mathcal{X}$.

Proposition 5.1. For $g=\left[\begin{array}{ll}a & b \\ 0 & a\end{array}\right]$, the $\left(\mathcal{F}_{g}, \mathcal{A}\right)$-equivalence $\mathcal{Z}_{g}=\mathbb{Z} \times \mathbb{T} \times \mathbb{R}$ is given by:

$$
\begin{aligned}
\mathcal{F}_{g} \curvearrowright \mathcal{Z}_{g}: & \left(\left[\begin{array}{c}
v \\
0
\end{array}\right]+\left(\frac{l_{1}+l_{2} \theta}{b}+r\right)\left(\begin{array}{c}
\theta \\
1
\end{array}\right), l_{1}, l_{2}\right) \cdot(k,[v], r)=\left(k+l_{2},[v], \frac{l_{1}+l_{2} \theta}{b}+r\right), \\
\mathcal{Z}_{g} \curvearrowleft \mathcal{A}: & (k,[v], r) \cdot\left([v], k_{1},[a v+r b+k \theta], k_{2}\right)=\left(k+k_{2}-a k_{1},\left[v-k_{1} \theta\right], r+k_{1}\right) .
\end{aligned}
$$

Now, we will finally compute the module structure of $\mathcal{L}_{g}$, but only for matrices $g$ of the above form. This, in turn, will then allow us to give the formulas for the Hilbert module structure of $\mathcal{N}_{g}^{0} \cong\left(A_{\theta} \otimes \mathcal{L}_{g}\right) \otimes_{A_{\theta}^{\otimes 3}}\left(L^{2} \otimes A_{\theta}\right)$.

Let $g=\left[\begin{array}{ll}a & b \\ 0 & a\end{array}\right]$ : recall that $Z_{g}$ is the Morita equivalence built as completion of $C_{c}\left(\mathcal{Z}_{g}\right)$, and by 'forgetting' its left-action, we arrived at the right- $A_{\theta} \otimes A_{\theta}$-Hilbert module $\mathcal{L}_{g}=\iota^{*}\left(Z_{g}\right)$. This means that their right Hilbert-module structures coincide, and so according to Theorem 2.8 in [20], the right- $C_{c}(\mathcal{A})$-action on the dense subspace $C_{c}\left(\mathcal{Z}_{g}\right)$ of $\mathcal{L}_{g}$ needs to be defined by

$$
\left(\Phi \cdot \mathcal{L}_{g} f\right)(\mathbf{z})=\int_{\substack{\text { sensible } \\ \nu \in \mathcal{A}}} \Phi(\mathbf{z} \cdot \nu) f\left(\nu^{-1}\right) \mathrm{d} \nu,
$$

where $\nu$ is "sensible" if $\mathbf{z} . \nu$ makes sense. For $\mathbf{z}=(k,[v], r) \in \mathcal{Z}_{g}$, this is the case exactly when $\nu=\left([v],-k_{1},[a v+r b-k \theta],-k_{2}\right)$ for some $k_{i} \in \mathbb{Z}$, in which case

$$
\mathbf{z} . \nu=\left(k-k_{2}+a k_{1},\left[v+k_{1} \theta\right], r-k_{1}\right) .
$$

The inverse of such $\nu$ in $\mathcal{A}_{\theta} \times \mathcal{A}_{\theta}$ is $\nu^{-1}=\left(\left[v+k_{1} \theta\right], k_{1},\left[a v+r b+\left(k_{2}-k\right) \theta\right], k_{2}\right)$. All in all this means:

$$
\begin{aligned}
\left(\Phi \cdot \mathcal{L}_{g} f\right)(k,[v], r)=\sum_{k_{1}, k_{2} \in \mathbb{Z}} \Phi\left(k-k_{2}+a k_{1},\left[v+k_{1} \theta\right], r-k_{1}\right) \\
\quad f\left(\left[v+k_{1} \theta\right], k_{1},\left[a v+r b+\left(k_{2}-k\right) \theta\right], k_{2}\right) .
\end{aligned}
$$


In particular, for $f=V^{l_{1}} U^{k_{1}} \otimes V^{l_{2}} U^{k_{2}}$ :

$$
\begin{aligned}
\left(\Phi \cdot \mathcal{L}_{g} V^{l_{1}} U^{k_{1}} \otimes V^{l_{2}} U^{k_{2}}\right)(k,[v], r)= & \Phi\left(k-k_{2}+a k_{1},\left[v+k_{1} \theta\right], r-k_{1}\right) \\
& \mathrm{e}^{2 \pi i l_{1}\left(v+k_{1} \theta\right)} \mathrm{e}^{2 \pi i l_{2}\left(a v+r b+\left(k_{2}-k\right) \theta\right)} .
\end{aligned}
$$

Now that we have concrete formulas for the right-action on $\mathcal{L}_{g}$, we can make the structure of $\mathcal{N}_{g}^{0}$ concrete by using Formula (5.6):

$$
\begin{array}{ll}
\mathfrak{A} \curvearrowright \mathcal{N}_{g}^{0}: & \left(V^{l_{1}} U^{k_{1}} \square \Phi\right)(k,[v], r)=\Phi\left(k-a k_{1},\left[v-k_{1} \theta\right], r+k_{1}\right) \mathrm{e}^{2 \pi i l_{1} v}, \\
\mathcal{N}_{g}^{0} \curvearrowleft \mathfrak{A}: & \left(\Phi \square V^{l_{2}} U^{k_{2}}\right)(k,[v], r)=\lambda^{l_{2}\left(k_{2}-k\right)} \Phi\left(k-k_{2},[v], r\right) \mathrm{e}^{2 \pi i l_{2}(a v+r b)} .
\end{array}
$$

We now compare this right-module structure of $\mathcal{N}_{g}^{0}$ to the right-module structure it would have if it came via descent from a suitable (yet to be determined) completion of $C_{c}(\mathbb{T} \times \mathbb{R})$ : for any $l_{2}, k_{2} \in \mathbb{Z},(k,[v], r) \in \mathcal{Z}_{g}=\mathbb{Z} \times \mathbb{R} \times \mathbb{T}$, and $\Phi \in C_{c}\left(\mathcal{Z}_{g}\right)$, we would need

$$
\lambda^{l_{2}\left(k_{2}-k\right)} \Phi\left(k-k_{2},[v], r\right) \mathrm{e}^{2 \pi i l_{2}(a v+r b)} \stackrel{!}{=}\left(\lambda^{l_{2}\left(k_{2}-k\right)} \Phi\left(k-k_{2}\right) * z^{l_{2}}\right)([v], r) .
$$

Here, the left-hand side is the right-action by $V^{l_{2}} U^{k_{2}}$ on the function $\Phi$, an element of the dense subspace $C_{c}\left(\mathcal{Z}_{g}\right)$ of $\mathcal{N}_{g}^{0}$. The right-hand side is the formula for the right-action by $V^{l_{2}} U^{k_{2}}$ as 'prescribed' by descent; notice that $\Phi\left(k-k_{2}\right)$ is our notation for the function

$$
\mathbb{T} \times \mathbb{R} \ni([v], r) \mapsto \Phi\left(k-k_{2},[v], r\right)
$$

in $C_{c}(\mathbb{T} \times \mathbb{R})$. In other words, if we define for $\phi \in C_{c}(\mathbb{T} \times \mathbb{R})$ and $f \in C(\mathbb{T})$,

$$
(\phi * f)([v], r)=\phi([v], r) f([a v+r b]),
$$

then descent turns this right-action of $C(\mathbb{T})$ on (a completion of ) $C_{c}(\mathbb{T} \times \mathbb{R})$ into the right-module structure we have on $\mathcal{N}_{g}^{0}$.

For the left-module structure to be coming from descent, we similarly require for any $l_{1}, k_{1} \in \mathbb{Z}$ that

$$
\Phi\left(k-a k_{1},\left[v-k_{1} \theta\right], r+k_{1}\right) \mathrm{e}^{2 \pi i l_{1} v} \stackrel{!}{=}\left(z^{l_{1}} *\left(k_{1} . \Phi\left(k-k_{1}\right)\right)\right)([v], r) .
$$

This shows that we need to have $a=1$, so that we can define for $\phi \in C_{c}(\mathbb{T} \times \mathbb{R})$ the action of $k_{1} \in \mathbb{Z}$ and the left-action of $f \in C(\mathbb{T})$ by:

$$
\left(k_{1} . \phi\right)([v], r)=\phi\left(\left[v-k_{1} \theta\right], r+k_{1}\right) \quad \text { and } \quad(f * \phi)([v], r)=f([v]) \phi([v], r) .
$$

For $g=\left[\begin{array}{ll}1 & b \\ 0 & 1\end{array}\right]$ with $b \in \mathbb{Z}^{\times}$, the inner products of both $\mathcal{L}_{b}=\mathcal{L}_{g}$ and subsequently of $\mathcal{N}_{b}^{0}:=\mathcal{N}_{g}^{0}$ are now easy to compute. First, the $A_{\theta} \otimes A_{\theta}$-valued inner product of $\mathcal{L}_{b}=\iota^{*}\left(Z_{g}\right)$ is just the inner product of $Z_{g}$. Therefore, Theorem 2.8 in [20] gives us the following formula for the inner product of two functions $\Phi, \Psi \in C_{c}\left(\mathcal{Z}_{g}\right) \subseteq \mathcal{L}_{b}$ evaluated at $\nu \in \mathcal{A}=\mathcal{A}_{\theta} \times \mathcal{A}_{\theta}$ :

$$
\langle\Phi \mid \Psi\rangle^{\mathcal{L}_{b}}(\nu)=\int_{\substack{\text { sensible } \\ \gamma \in \mathcal{F}_{g}}} \bar{\Phi}(\gamma \cdot \mathbf{z}) \Psi(\gamma \cdot \mathbf{z} . \nu) \mathrm{d} \gamma,
$$

where $\mathbf{z} \in \mathcal{Z}_{g}=\mathbb{Z} \times \mathbb{R} \times \mathbb{T}$ is any element such that $\mathbf{z} . \nu$ makes sense in $\mathcal{Z}_{g}$, and $\gamma$ is "sensible" if $\gamma . \mathbf{z}$ is defined. According to Proposition 5.1 when $\nu=\left([v], l_{1},[w], l_{2}\right)$, 
we can take the element $\mathbf{z}=\left(0,[v], \frac{w-v}{b}\right)$ for some choice of representatives $v, w$ of $[v, w]$. For sensible $\gamma \in \mathcal{F}_{g}$, we have

$$
\gamma \cdot \mathbf{z}=\left(k_{2},[v], \frac{k_{1}+k_{2} \theta+w-v}{b}\right),
$$

where $k_{1}, k_{2} \in \mathbb{Z}$ are arbitrary, and then

$$
\gamma . \mathbf{z} . \nu=\left(k_{2}+l_{2}-l_{1},\left[v-l_{1} \theta\right], \frac{k_{1}+k_{2} \theta+w-v}{b}+l_{1}\right) .
$$

All in all:

$$
\begin{aligned}
& \langle\Phi \mid \Psi\rangle^{\mathcal{L}_{b}}\left([v], l_{1},[w], l_{2}\right) \\
& =\sum_{k_{1}, k_{2} \in \mathbb{Z}} \bar{\Phi}\left(k_{2},[v], \frac{k_{1}+k_{2} \theta+w-v}{b}\right) \Psi\left(k_{2}+l_{2}-l_{1},\left[v-l_{1} \theta\right], \frac{k_{1}+k_{2} \theta+w-v}{b}+l_{1}\right) .
\end{aligned}
$$

Now we will use Lemma 5.3 to compute a formula for $\langle\Phi \mid \Psi\rangle^{\mathcal{N}_{b}}$ where $\Phi, \Psi \epsilon$ $C_{c}\left(\mathcal{Z}_{g}\right) \subseteq \mathcal{N}_{g}=\mathcal{N}_{b}:$

$$
\begin{aligned}
\langle\Phi \mid \Psi\rangle^{\mathcal{N}_{b}}([x], l) & =\int_{\mathbb{T}} \sum_{k_{1}, k_{2} \in \mathbb{Z}} \bar{\Phi}\left(k_{2},[y], \frac{k_{1}+k_{2} \theta+x-y}{b},\right) \Psi\left(k_{2}+l,[y], \frac{k_{1}+k_{2} \theta+x-y}{b}\right) \mathrm{d} y \\
& =\int_{\mathbb{R}} \sum_{k \in \mathbb{Z}} \bar{\Phi}\left(k,[r], \frac{k \theta+x-r}{b}\right) \Psi\left(k+l,[r], \frac{k \theta+x-r}{b}\right) \mathrm{d} r \\
& =\int_{\mathbb{R}} \sum_{k \in \mathbb{Z}} \bar{\Phi}\left(k,[x+k \theta-r], \frac{r}{b}\right) \Psi\left(k+l,[x+k \theta-r], \frac{r}{b}\right) \mathrm{d} r .
\end{aligned}
$$

For this to come from descent, we need

$$
\langle\Phi \mid \Psi\rangle^{\mathcal{N}_{b}}([x], l) \stackrel{!}{=} \sum_{k}\langle\Phi(k) \mid \Psi(k+l)\rangle_{C(\mathbb{T})}^{N_{g}}([x+k \theta]) .
$$

This is satisfied if we define

$$
\langle\phi \mid \psi\rangle_{C(\mathbb{T})}^{N_{b}^{0}}([x]):=\int_{\mathbb{R}}(\bar{\phi} \psi)\left([x-r], \frac{r}{b}\right) \mathrm{d} r .
$$

In Theorem 5.4 below, we will sum up what we have found so far, namely the formulas for the lift via descent of the module $\mathcal{N}_{b}^{0}$.

\subsection{Conclusion of the proof.}

Theorem 5.4. Suppose $b \in \mathbb{Z}^{\times}$. We define the structure of an equivariant, rightpre-Hilbert $C(\mathbb{T})$-bimodule on $C_{c}(\mathbb{T} \times \mathbb{R})$ by

$$
\begin{aligned}
\phi, \psi \in C_{c}(\mathbb{T} \times \mathbb{R}): & \langle\phi \mid \psi\rangle_{C(\mathbb{T})}([x]) & =\int_{\mathbb{R}}(\bar{\phi} \psi)\left([x-r], \frac{r}{b}\right) \mathrm{d} r, \\
\mathbb{Z} \curvearrowright C_{c}(\mathbb{T} \times \mathbb{R}): & (l . \phi)([x], r) & =\phi([x-l \theta], r+l), \\
C(\mathbb{T}) \curvearrowright C_{c}(\mathbb{T} \times \mathbb{R}): & (f * \phi)([x], r) & =f([x]) \phi([x], r), \\
C_{c}(\mathbb{T} \times \mathbb{R}) \curvearrowleft C(\mathbb{T}): & (\phi * f)([x], r) & =\phi([x], r) f([x+r b]) .
\end{aligned}
$$

Let $N_{b}^{ \pm}$be the completion of $C_{c}(\mathbb{T} \times \mathbb{R})$ with respect to this pre-inner product, and let $N_{b}:=N_{b}^{+} \oplus N_{b}^{-}$be standard evenly graded. Define the unbounded operator $d_{N_{b},+}: N_{b}^{+} \rightarrow N_{b}^{-}$by

$$
d_{N_{b},+}:=-\frac{1}{b} \frac{\partial}{\partial r}+\frac{\partial}{\partial \Theta}-2 \pi \mathrm{M}
$$

let $d_{N_{b},-}:=d_{N_{b},+}^{*}$ and define

$$
d_{N_{b}}:=\left[\begin{array}{cc}
0 & d_{N_{b},-} \\
d_{N_{b},+} & 0
\end{array}\right] .
$$

Then the pair $\left(N_{b}, d_{N_{b}}\right)$ is a cycle in $\Psi^{\mathbb{Z}}(C(\mathbb{T}), C(\mathbb{T}))$. 
Remark 5.5. To see why we chose this (pre-)Hilbert module structure, see Formula (5.13), Formula (5.12), and Formula (5.15). To see why we chose this operator, see the proof of Lemma 5.7

To prove Theorem [5.4 we will check that $\left(N_{b}, b \cdot d_{N_{b}}\right)$ is unitarily equivalent to the equivariant cycle $\left(H_{b}, \mathrm{id}_{C(\mathbb{T})} \otimes d_{\lambda}\right)$ of Remark 4.6 for $\lambda:=2 \pi b \in \mathbb{R}^{\times}$. Recall that we defined $H_{b}^{ \pm}$as the completion of $C_{c}(\mathbb{T} \times \mathbb{R})$ with respect to the pre-Hilbert module structure given on page 263 which is also where the definition of $d_{\lambda}$ can be found. Note that the domain of $\operatorname{id}_{C(\mathbb{T})} \otimes d_{\lambda}$ contains, by definition, the subspace $C(\mathbb{T}) \odot \mathcal{S}(\mathbb{R})$.

Proof of Theorem [5.4. Define $w: \mathbb{T} \times \mathbb{R} \longrightarrow \mathbb{T} \times \mathbb{R}$ by

$$
w([x], r):=([x+b r],-r),
$$

so that $w=w^{-1}$, and let

$$
\begin{array}{cc}
H_{b}^{ \pm} \supseteq C_{c}(\mathbb{T} \times \mathbb{R}) \underset{W}{\longleftarrow} C_{c}(\mathbb{T} \times \mathbb{R}) \subseteq N_{b}^{ \pm} \\
W^{-1} \phi:=\sqrt{|b|} \cdot \phi \circ w & W \phi:=\frac{1}{\sqrt{|b|}} \cdot \phi \circ w
\end{array}
$$

It is quickly checked that this induces the claimed structure on $H_{b}^{ \pm}$.

Finally, a routine computation shows that

$$
\begin{aligned}
W^{-1} \circ b \cdot d_{N_{b},+} \circ W & \stackrel{\sqrt{5.16}}{=} W^{-1} \circ\left(-\frac{\partial}{\partial r}+b \frac{\partial}{\partial \Theta}-2 \pi b \mathrm{M}\right) \circ W \\
& =-\left(b \frac{\partial}{\partial \Theta}-\frac{\partial}{\partial r}\right)+b \frac{\partial}{\partial \Theta}+2 \pi b \mathrm{M} \\
& =2 \pi b \mathrm{M}+\frac{\partial}{\partial r}=d_{2 \pi b,+}
\end{aligned}
$$

as claimed.

Since we have proved $\left(H_{b}, d_{\lambda}\right)$ to be an unbounded cycle for any $\lambda \in \mathbb{R}^{\times}$(see Theorem 4.1), it follows that $\left(N_{b}, b \cdot d_{N_{b}}\right)$ and hence $\left(N_{b}, d_{N_{b}}\right)$ are cycles also.

We will next verify that $\left(\mathcal{N}_{b}, D_{\mathcal{N}_{b}}\right)$ satisfies all properties needed to invoke [15, Theorem 13], the well-known recipe due to Kucerovsky how to determine that a given unbounded KK-cycle is the Kasparov product of two other cycles.

Theorem 5.6. For $\left(N_{b}, d_{N_{b}}\right)$ as defined in Theorem 5.4 and

$$
j: \Psi_{0}^{\mathbb{Z}}(C(\mathbb{T}), C(\mathbb{T})) \longrightarrow \Psi_{0}\left(A_{\theta}, A_{\theta}\right)
$$

the descent map, the cycle $j\left(N_{b}, d_{N_{b}}\right)=\left(\mathcal{N}_{b}, D_{\mathcal{N}_{b}}\right)$ represents the Kasparov product $\left(1_{A_{\theta}} \otimes\left[\mathcal{L}_{b}\right]\right) \otimes_{A_{\theta}^{\otimes 3}}\left(\Delta_{\theta} \otimes 1_{A_{\theta}}\right)$.

We have already found that the module $N_{b}$ descends to $\mathcal{N}_{b}=\mathcal{N}_{b}^{0} \oplus \mathcal{N}_{b}^{0}$ - in fact, this is where the formulas that we used to define $N_{b}$ came from, see Formula (5.13), Formula (5.12), and Formula (5.15). Furthermore, we have seen that $\mathcal{N}_{b}^{ \pm}$ can be regarded as $\left(A_{\theta} \otimes \mathcal{L}_{b}\right) \otimes_{A_{\theta}^{\otimes 3}}\left(L^{2} \otimes A_{\theta}\right)$, two copies of which make up the module underlying $\left(1_{A_{\theta}} \otimes\left[\mathcal{L}_{b}\right]\right) \otimes_{A_{\theta}^{\otimes 3}}\left(\Delta_{\theta} \otimes 1_{A_{\theta}}\right)$ via Equations (5.2) and (5.5). The identification can be summed up as follows:

$$
\underbrace{\left(V^{l_{1}} U^{k_{1}} \otimes \Phi\right) \otimes_{B}\left(\left(z^{l_{2}} \otimes \varepsilon_{k_{2}}\right) \otimes V^{l_{3}} U^{k_{3}}\right)}_{\in\left(A_{\theta} \otimes \mathcal{L}_{b}\right) \otimes_{A_{\theta}^{\otimes 3}}\left(L^{2} \otimes A_{\theta}\right)} \triangleq \lambda^{l_{1}\left(k_{1}+k_{2}\right)} \underbrace{\Phi \cdot \mathcal{L}_{b}\left(V^{l_{1}+l_{2}} U^{-\left(k_{1}+k_{2}\right)} \otimes V^{l_{3}} U^{k_{3}}\right)}_{\in \mathcal{N}_{b}^{ \pm}}
$$


We have also already proved that $\left(\mathcal{N}_{b}, D_{\mathcal{N}_{b}}\right)$ is indeed in $\Psi\left(A_{\theta}, A_{\theta}\right)$. Therefore, we now only need to prove the following:

Lemma 5.7. For all $x$ in a dense subset of $A_{\theta} \otimes \mathcal{L}_{b}$, the operator

$$
\left[\left[\begin{array}{cc}
D_{\mathcal{N}_{b}} & 0 \\
0 & d_{\Delta} \otimes 1
\end{array}\right],\left[\begin{array}{cc}
0 & T_{x} \\
T_{x}^{*} & 0
\end{array}\right]\right]
$$

extends to a bounded operator.

We note that $C_{c}^{\infty}\left(\mathcal{A}_{\theta}\right) \odot C_{c}^{\infty}\left(\mathcal{Z}_{b}\right)$ is dense in $A_{\theta} \otimes \mathcal{L}_{b}$ by the following:

Lemma 5.8. Suppose $\Phi_{n} \in C_{c}(\mathbb{Z} \times \mathbb{T} \times \mathbb{R})$ are such that $\left\|\Phi_{n}\right\|_{\infty} \stackrel{n \rightarrow \infty}{\longrightarrow} 0$ and that, for all $n$, the support of $\Phi_{n}$ is contained in some compact set. Then $\Phi_{n} \stackrel{n \rightarrow \infty}{\longrightarrow} 0$ both in $\mathcal{L}_{b}$ and in $\mathcal{H}_{-b}^{ \pm}$.

The proof of Lemma 5.8 employs a "standard trick" that was used in the proof of Theorem 2.8 in [20]: the inductive limit topology on $C_{c}(\mathcal{G})$ for $\mathcal{G}$ a second countable locally compact Hausdorff étale groupoid is finer than the topology given by the $\mathrm{C}^{*}$-norm (see [23. Chapter II, Proposition 1.4(i)]).

Corollary 5.9. If $c_{00}$ denotes the space of bi-infinite sequences which are eventually zero, then the subspace $c_{00} \odot \operatorname{span}\left\{z^{n} \mid n \in \mathbb{Z}\right\} \odot C_{c}^{\infty}(\mathbb{R})$ is dense in both $\mathcal{L}_{b}$ and $\mathcal{H}_{-b}^{ \pm}$.

Remark 5.10. The statement in Lemma 5.7 implicitly makes use of the identification in Equation (5.19). In other words, our claim (for the creation part) can be rephrased to saying that the following diagram is commutative up to adjointable operators, where $\mathcal{N}^{1}:=A_{\theta} \otimes\left[\mathcal{L}_{b}\right]$ and $\mathcal{N}^{2}:=\left(L^{2} \oplus L^{2}\right) \otimes A_{\theta}$ :

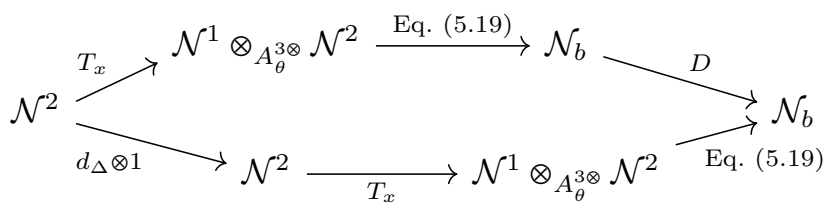

We observe that only the creation-part in Lemma 5.7 has to be shown.

Lemma 5.11. Let $D: \operatorname{Dom}(D) \rightarrow \mathcal{N}$ be a self-adjoint, densely defined unbounded operator on a right-Hilbert $C^{*}$-module $\mathcal{N}$ over some $C^{*}$-algebra $C$. Let $T \in \mathcal{L}(\mathcal{N})$ be such that $\operatorname{Dom}(D T) \cap \operatorname{Dom}(D)$ and $\operatorname{Dom}(D) \cap \operatorname{Dom}\left(D T^{*}\right)$ are dense. If the operator DT+TD (or DT-TD) extends to a bounded operator, then its extension is adjointable and $T^{*} D+D T^{*}$ (resp. $\left.T^{*} D-D T^{*}\right)$ also extends to an adjointable operator.

Proof. Let $S:=T^{*} D \pm D T^{*}$ and $R:=D T \pm T D$, so that $\operatorname{Dom}(S)=\operatorname{Dom}(D) \cap$ $\operatorname{Dom}\left(D T^{*}\right)$ and $\operatorname{Dom}(R)=\operatorname{Dom}(D T) \cap \operatorname{Dom}(D)$ are dense by assumption. We claim that $R^{*}$ extends $S$ and that it is an adjointable operator.

We compute for $\xi \in \operatorname{Dom}(S)$ and $\zeta \in \operatorname{Dom}(R)$

$$
\begin{aligned}
\langle R \zeta \mid \xi\rangle & =\langle D T \zeta \mid \xi\rangle \pm\langle T D \zeta \mid \xi\rangle=\langle T \zeta \mid D \xi\rangle \pm\left\langle D \zeta \mid T^{*} \xi\right\rangle \\
& =\left\langle\zeta \mid T^{*} D \xi\right\rangle \pm\left\langle\zeta \mid D T^{*} \xi\right\rangle=\langle\zeta \mid S \xi\rangle .
\end{aligned}
$$

This shows that $\operatorname{Dom}(S)$ is a subset of

$$
\operatorname{Dom}\left(R^{*}\right)=\{\xi \in \mathcal{N} \mid \exists y \in \mathcal{N}, \forall \zeta \in \operatorname{Dom}(R):\langle R \zeta \mid \xi\rangle=\langle\zeta \mid y\rangle\}
$$


Moreover, for any $\zeta \in \operatorname{Dom}(R)$ and $\xi \in \operatorname{Dom}(S)$,

$$
\langle\zeta \mid S \xi\rangle=\langle R \zeta \mid \xi\rangle=\left\langle\zeta \mid R^{*} \xi\right\rangle \text {. }
$$

We know that this property uniquely defines $R^{*} \xi$ since $\operatorname{Dom}(R)$ is dense, and hence $R^{*} \xi=S \xi$ on $\operatorname{Dom}(S)$. In other words, $R^{*}$ extends $S$. In particular, $R^{*}$ is also densely defined.

Let $\bar{R}$ be the assumed bounded extension of $R$. Then for $\zeta \in \operatorname{Dom}(R)$ and $\xi \in \operatorname{Dom}\left(R^{*}\right)$, we have

$$
\left\langle\zeta \mid R^{*} \xi\right\rangle=\langle R \zeta \mid \xi\rangle=\langle\bar{R} \zeta \mid \xi\rangle \text {, so that }\left\|\left\langle\zeta \mid R^{*} \xi\right\rangle\right\| \leq\|\bar{R}\| \cdot\|\zeta\| \cdot\|\xi\| .
$$

As $\operatorname{Dom}(R)$ is dense, we conclude that the norm inequality in (5.21) holds for all $\zeta \in \mathcal{N}$. In particular, choosing $\zeta=R^{*} \xi$ yields $\left\|R^{*}(\xi)\right\| \leq\|\bar{R}\| \cdot\|\xi\|$, so the closed operator $R^{*}$ is bounded by $\|\bar{R}\|$ on its entire dense domain. This implies that $R^{*}$ is a bounded operator. Using denseness of $\operatorname{Dom}(R)$ once again and $\operatorname{Dom}\left(R^{*}\right)=\mathcal{N}$, Equation (5.21) shows that $(\bar{R})^{*}=R^{*}$, so that $\bar{R}$ and $R^{*}$ are adjointable operators, as claimed.

Corollary 5.12. Let $D: \operatorname{Dom}(D) \rightarrow \mathcal{N}$ and $D^{\prime}: \operatorname{Dom}\left(D^{\prime}\right) \rightarrow \mathcal{N}^{\prime}$ be two self-adjoint, densely defined unbounded operators on right-Hilbert $C^{*}$-modules $\mathcal{N}$ resp. $\mathcal{N}^{\prime}$ over some $C^{*}$-algebra $C$. Let $T \in \mathcal{L}\left(\mathcal{N}^{\prime}, \mathcal{N}\right)$ be such that $\operatorname{Dom}(D T) \cap \operatorname{Dom}\left(D^{\prime}\right)$ and $\operatorname{Dom}(D) \cap \operatorname{Dom}\left(D^{\prime} T^{*}\right)$ are dense. If the operator $D T+T D^{\prime}$ (or $D T-T D^{\prime}$ ) extends to a bounded operator, then its extension is adjointable and $T^{*} D+D^{\prime} T^{*}$ (resp. $T^{*} D-D^{\prime} T^{*}$ ) also extends to an adjointable operator.

Proof. Consider the self-adjoint, densely defined operator $\mathcal{D}:=\left[\begin{array}{cc}D & 0 \\ 0 & D^{\prime}\end{array}\right]$ and the adjointable operator $\mathcal{T}:=\left[\begin{array}{ll}0 & T \\ 0 & 0\end{array}\right]$ on the right-Hilbert $C^{*}$-module $\mathcal{N} \oplus \mathcal{N}^{\prime}$. Then

$$
\operatorname{Dom}(\mathcal{D} \mathcal{T}) \cap \operatorname{Dom}(\mathcal{D})=\operatorname{Dom}(D) \oplus\left(\operatorname{Dom}(D T) \cap \operatorname{Dom}\left(D^{\prime}\right)\right)
$$

and

$$
\operatorname{Dom}(\mathcal{D}) \cap \operatorname{Dom}\left(\mathcal{D} \mathcal{T}^{*}\right)=\left(\operatorname{Dom}(D) \cap \operatorname{Dom}\left(D^{\prime} T^{*}\right)\right) \oplus \operatorname{Dom}\left(D^{\prime}\right)
$$

are both dense by assumption. Since $\mathcal{D} \mathcal{T} \pm \mathcal{T} \mathcal{D}=\left[\begin{array}{cc}0 & D T \pm T D^{\prime} \\ 0 & 0\end{array}\right]$ extends to a bounded operator by assumption, we may use Lemma 5.11 and the claim follows.

Proof of Lemma 5.7. We start by considering an elementary tensor $x=a \otimes \Phi$ in $C_{c}^{\infty}\left(\mathcal{A}_{\theta}\right) \odot C_{c}^{\infty}\left(\mathcal{Z}_{b}\right)$.

Let us untangle Diagram (5.20) and be precise: Instead of working with $D_{\mathcal{N}_{b}}$ on $\mathcal{N}_{b}$, we will work with the corresponding operator $\tilde{D}$ on the actual space $\mathcal{N}^{1} \otimes_{A_{\theta}^{3 \otimes}} \mathcal{N}^{2}$ (using Equation (5.19) to figure out $\tilde{D}$ ). Unfortunately, $\tilde{D}$ is going to be very unwieldy, which is the reason we instead chose to define $D$ 's lift in Theorem [5.4] The upshot is that $\tilde{D} T_{x}-T_{x}\left(d_{\Delta} \otimes 1\right)$ will turn out to be extended by a creation operator, which is clearly adjointable.

Note that, since $C_{c}^{\infty}\left(\mathcal{Z}_{b}\right)$ is a subspace of $\operatorname{Dom}(D)$ which contains $C_{c}^{\infty}\left(\mathcal{Z}_{b}\right) \cdot_{\mathcal{L}_{b}}\left(C_{c}^{\infty}\left(\mathcal{A}_{\theta}\right)\right)^{2 \odot}$, the map in Equation (5.19) shows that

$$
\left(C_{c}^{\infty}\left(\mathcal{A}_{\theta}\right) \odot C_{c}^{\infty}\left(\mathcal{Z}_{b}\right)\right) \odot_{\mathfrak{A}^{3} \odot}\left(C_{c}^{\infty}\left(\mathcal{A}_{\theta}\right) \odot C_{c}^{\infty}\left(\mathcal{A}_{\theta}\right)\right) \subseteq \operatorname{Dom}\left(\tilde{D}_{ \pm}\right) .
$$

Consequently, $T_{x}$ for $x=a \otimes \Phi$ as above maps $C_{c}^{\infty}\left(\mathcal{A}_{\theta}\right) \odot C_{c}^{\infty}\left(\mathcal{A}_{\theta}\right)$ into $\operatorname{Dom}\left(\tilde{D}_{ \pm}\right)$. Note that $C_{c}^{\infty}\left(\mathcal{A}_{\theta}\right) \odot C_{c}^{\infty}\left(\mathcal{A}_{\theta}\right)$ is also contained in the domain of $D_{2, \pm}:=d_{\Delta, \pm} \otimes 1$, so in particular, $\operatorname{Dom}\left(\tilde{D}_{ \pm} T_{x}\right) \cap \operatorname{Dom}\left(D_{2, \pm}\right)$ contains this dense subset of $\left(L^{2} \oplus L^{2}\right)^{ \pm} \otimes A_{\theta}$. 
For $a \in C_{c}^{\infty}\left(\mathcal{A}_{\theta}\right) \subseteq A_{\theta}$ and $f \in C_{c}^{\infty}\left(\mathcal{A}_{\theta}\right) \subseteq\left(L^{2} \oplus L^{2}\right)^{ \pm}$, define the function $\psi(a, f) \in$ $C_{c}^{\infty}\left(\mathcal{A}_{\theta}\right) \subseteq A_{\theta}$ by

$$
\psi(a, f)([x], k):=\sum_{n \in \mathbb{Z}} a([x-k \theta],-n) f([x], n-k),
$$

then for $a=V^{l_{1}} U^{k_{1}}$ and $f=z^{l_{2}} \otimes \varepsilon_{k_{2}}$, we recover $\psi(a, f)=\lambda^{l_{1}\left(k_{1}+k_{2}\right)} V^{l_{1}+l_{2}} U^{-\left(k_{1}+k_{2}\right)}$. This shows that, for $c \in C_{c}^{\infty}\left(\mathcal{A}_{\theta}\right)$ and $\Phi \in C_{c}^{\infty}\left(\mathcal{Z}_{b}\right)$, the map in Equation (5.19) identifies

$$
\begin{gathered}
\mathcal{N}^{1} \otimes_{A_{\theta}^{3 \otimes}}\left(\mathcal{N}^{2}\right)^{ \pm} \longleftrightarrow \mathcal{N}_{b}^{ \pm} \\
(a \otimes \Phi) \otimes_{A_{\theta}^{3 \otimes}}(f \otimes c) \triangleq \Phi \cdot \mathcal{L}_{b}(\psi(a, f) \otimes c)
\end{gathered}
$$

To find $\tilde{D}_{ \pm}\left((a \otimes \Phi) \otimes_{A_{\theta}^{3 \otimes}}(f \otimes c)\right)$, we see from Equation (5.23) that we first need to compute $D_{\mathcal{N}_{b}, \pm}\left(\Phi \cdot \mathcal{L}_{b}(\psi(a, f) \otimes c)\right)$. Note that, if $\xi:=\psi(a, f)$ and $\Psi:=\Phi \cdot \mathcal{L}_{b} \xi$, then Equation (5.9) (the formula for the right action on $\mathcal{L}_{b}$ ) reveals that

$$
\begin{aligned}
\frac{\partial \Psi}{\partial r} & =\left(\frac{\partial \Phi}{\partial r}\right) \cdot \mathcal{L}_{b} \xi+b \Phi \cdot \mathcal{L}_{b}\left(\psi(a, f) \otimes \frac{\partial c}{\partial \Theta}\right), \\
\frac{\partial \Psi}{\partial \Theta} & =\left(\frac{\partial \Phi}{\partial \Theta}\right) \cdot \mathcal{L}_{b} \xi+\Phi \cdot \mathcal{L}_{b}\left(\psi(a, f) \otimes \frac{\partial c}{\partial \Theta}+\frac{\partial \psi(a, f)}{\partial \Theta} \otimes c\right), \quad \text { and } \\
\mathrm{M}^{\mathbb{R}} \Psi & =\left(\mathrm{M}^{\mathbb{R}} \Phi\right) \cdot \mathcal{L}_{b} \xi+\Phi \cdot \mathcal{L}_{b}\left(\left(\mathrm{M}^{\mathbb{Z}} \psi(a, f)\right) \otimes c\right),
\end{aligned}
$$

where $M^{\mathbb{R}}$ resp. $M^{\mathbb{Z}}$ denotes the operator that multiplies by the input of the $\mathbb{R}$ - resp. the $\mathbb{Z}$-component, and $\frac{\partial}{\partial r}$ resp. $\frac{\partial}{\partial \Theta}$ refers to differentiation with respect to the $\mathbb{R}$ resp. $\mathbb{T}$-component.

It follows that, applying the operator $D:=D_{\mathcal{N}_{b}}$ on $\mathcal{N}_{b}$-built out of $d_{N_{b}}$ (see Definition 5.16) via descent- to $\Psi$ yields

$$
\begin{aligned}
D_{ \pm}(\Psi)=[\mp & \left.\frac{1}{b} \frac{\partial}{\partial r} \pm \frac{\partial}{\partial \Theta}-2 \pi \mathrm{M}^{\mathbb{R}}\right](\Phi) \cdot_{\mathcal{L}_{b}} \xi \\
& +\Phi \cdot \mathcal{L}_{b}\left[\psi\left( \pm \frac{\partial a}{\partial \Theta}+2 \pi \mathrm{M}^{\mathbb{Z}} a, f\right)+\psi\left(a, \pm \frac{\partial f}{\partial \Theta}+2 \pi \mathrm{M}^{\mathbb{Z}} f,\right)\right] \otimes c .
\end{aligned}
$$

This element corresponds via Equation (5.23) to the following element in $\mathcal{N}^{1} \otimes_{A_{\theta}^{3 \otimes}}$ $\left(\mathcal{N}^{2}\right)^{ \pm}$:

$$
\begin{aligned}
& \tilde{D}_{ \pm}\left((a \otimes \Phi) \otimes_{A_{\theta}^{3 \otimes}}(f \otimes c)\right) \\
& \quad:=\left(a \otimes\left[-2 \pi \mathrm{M}^{\mathbb{R}} \mp \frac{1}{b} \frac{\partial}{\partial r} \pm \frac{\partial}{\partial \Theta}\right](\Phi)+\left[2 \pi \mathrm{M}^{\mathbb{Z}} \pm \frac{\partial}{\partial \Theta}\right](a) \otimes \Phi\right) \otimes_{A_{\theta}^{3 \otimes}}(f \otimes c) \\
& \quad+(a \otimes \Phi) \otimes_{A_{\theta}^{3 \otimes}}\left(\left[2 \pi \mathrm{M}^{\mathbb{Z}} \pm \frac{\partial}{\partial \Theta}\right](f) \otimes c\right)
\end{aligned}
$$

Since $D_{\mathbb{T}}=-i \frac{\partial}{\partial \Theta}$ and $D_{\mathbb{Z}}=2 \pi \mathrm{M}^{\mathbb{Z}}$ (defined in Lemma (2.2) $)$, we get

$$
\left[2 \pi \mathrm{M}^{\mathbb{Z}} \pm \frac{\partial}{\partial \Theta}\right](f) \otimes x=\left(D_{\mathbb{Z}} \pm i D_{\mathbb{T}}\right)(f) \otimes c=\left(d_{\Delta_{\theta}, \pm} \otimes 1\right)(f \otimes c)=D_{2, \pm}(f \otimes c) .
$$

Thus, if we define for $x=a \otimes \Phi$ :

$$
X_{ \pm}(x):=a \otimes\left[-2 \pi \mathrm{M}^{\mathbb{R}} \mp \frac{1}{b} \frac{\partial}{\partial r} \pm \frac{\partial}{\partial \Theta}\right](\Phi)+\left[2 \pi \mathrm{M}^{\mathbb{Z}} \pm \frac{\partial}{\partial \Theta}\right](a) \otimes \Phi \in C_{c}^{\infty}\left(\mathcal{A}_{\theta}\right) \odot C_{c}^{\infty}\left(\mathcal{Z}_{b}\right),
$$


then this shows that

$$
\left.\tilde{D}_{ \pm}\left(T_{x}(f \otimes c)\right)=T_{X_{ \pm}(x)}(f \otimes c)+T_{x} D_{2, \pm}(f \otimes c)\right) .
$$

We conclude that $\tilde{D}_{ \pm} T_{x}-T_{x} D_{2, \pm}=T_{X_{ \pm}(x)}$ extends to an adjointable operator for $x$ any elementary tensor in $C_{c}^{\infty}\left(\mathcal{A}_{\theta}\right) \odot C_{c}^{\infty}\left(\mathcal{Z}_{b}\right)$. By linearity, we conclude that $\tilde{D}_{ \pm} T_{x}-T_{x} D_{2, \pm}$ is densely defined and extends to an adjointable operator for any $x \in C_{c}^{\infty}\left(\mathcal{A}_{\theta}\right) \odot C_{c}^{\infty}\left(\mathcal{Z}_{b}\right)$.

To prove that $T_{x}^{*} \tilde{D}_{ \pm}-D_{2, \pm} T_{x}^{*}$ extends to an adjointable as well, we want to invoke Corollary 5.12. The only thing that remains to check is that $\operatorname{Dom}\left(\tilde{D}_{ \pm}\right) \cap$ $\operatorname{Dom}\left(D_{2, \pm} T_{x}^{*}\right)$ is dense. So let $y \in C_{c}^{\infty}\left(\mathcal{A}_{\theta}\right) \odot C_{c}^{\infty}\left(\mathcal{Z}_{b}\right)$ be another element like $x$ and let $F \in C_{c}^{\infty}\left(\mathcal{A}_{\theta}\right) \odot C_{c}^{\infty}\left(\mathcal{A}_{\theta}\right) \subseteq\left(L^{2} \oplus L^{2}\right)^{ \pm} \otimes A_{\theta}$. We have

$$
T_{x}^{*}(y \otimes(f \otimes c))=\langle x \mid y\rangle_{A_{\theta}^{\otimes 3}}^{A_{\theta} \otimes \mathcal{L}_{b}} \cdot F,
$$

where $\cdot$ denotes the action of $A_{\theta}^{\otimes 3}$ on $F$. One readily sees from Equation (5.14) (the formula for the $A_{\theta} \otimes A_{\theta}$-valued inner product on $\mathcal{L}_{b}$ ) that $\langle x \mid y\rangle_{A_{\theta}^{\otimes 3}}^{A_{\theta} \otimes \mathcal{L}_{b}}$ is not just a smooth but also a compactly supported function on $\mathcal{A}_{\theta} \times \mathcal{A}_{\theta} \times \mathcal{A}_{\theta}$. By bootstrapping from elementary tensors (similarly to how it was done in the proof of the inner product formula of Lemma 5.3), one finds the following formula for the $A_{\theta}^{\otimes 3}$-action on $L^{2} \otimes A_{\theta}$ that holds for any $a \in C_{c}^{\infty}\left(\mathcal{A}_{\theta} \times \mathcal{A}_{\theta} \times \mathcal{A}_{\theta}\right) \subseteq A_{\theta}^{\otimes 3}$ acting on the element $F$ :

$$
\begin{gathered}
(a \cdot F)\left([x], m_{1},[y], m_{2}\right)=\sum_{k, l, n} a\left(\left[x+m_{1} \theta\right], m_{1}+k-n,[x], k,[y], l\right) \\
F\left([x-k \theta], n,[y-l \theta], m_{2}-l\right) .
\end{gathered}
$$

Using that $a$ and $F$ are smooth and compactly supported, one sees that $a \cdot F$ is also smooth and compactly supported. In particular, this holds for $a=\langle x \mid y\rangle_{A_{\theta}^{\otimes 3}}^{A_{\theta} \otimes \mathcal{L}_{b}}$. Thus, $T_{x}^{*}$ maps the dense subset of $\operatorname{Dom}\left(\tilde{D}_{ \pm}\right)$from Equation (5.22) into the dense subset $C_{c}^{\infty}\left(\mathcal{A}_{\theta} \times \mathcal{A}_{\theta}\right)$ of $\operatorname{Dom}\left(D_{2, \pm}\right)$, proving the claim.

\section{Conclusion of the Duality theorem}

As a result of the previous sections, we have obtained the following, where we use that $\tau_{b}^{-1}=\tau_{-b}$ by Theorem 4.7 .

Theorem 6.1. Let $g=\left[\begin{array}{ll}1 & b \\ 0 & 1\end{array}\right]$ for $b>0$ and $\mathcal{L}_{b}:=\mathcal{L}_{g}$ (Definition 3.6). Then

$$
\left(1_{A_{\theta}} \otimes \tau_{-b}\right)_{*}\left(\left[\mathcal{L}_{b}\right]\right) \otimes_{A_{\theta}^{\otimes 3}}\left(\Delta_{\theta} \otimes 1_{A_{\theta}}\right)=1_{A_{\theta}},
$$

where $\tau_{b} \in \mathrm{KK}_{0}\left(A_{\theta}, A_{\theta}\right)$ is the b-twist (Definition 4.5). In particular, the class

$$
\widehat{\Delta}_{\theta}:=\left(1_{A_{\theta}} \otimes \tau_{-b}\right)_{*}\left(\mathcal{L}_{b}\right)
$$

together with Connes' class $\Delta_{\theta}$, satisfy the zig-zag equations. The classes $\Delta_{\theta}, \widehat{\Delta}_{\theta}$ are the co-unit and unit, respectively, of a self-duality for $A_{\theta}$.

We have proved the first zig-zag equation, and the second follows in exactly the same way; we omit the details to avoid much duplication. We can now describe a spectral cycle representative for $\widehat{\Delta}_{\theta}$. First, recall that $\tau_{-b}$ can be described as the descended version of the cycle $\left(H_{-b}, d_{1}\right)$, i.e.

$$
\tau_{-b}=j\left(\left[\left(H_{-b}, d_{1}\right)\right]\right)=:\left[\left(\mathcal{H}_{-b}, D_{1}\right)\right],
$$


Thus, its module is a completion of $C_{c}(\mathbb{Z} \times \mathbb{T} \times \mathbb{R})$, described explicitly in Lemma 4.4 and its operator $D_{1}=\left[\begin{array}{cc}0 & D_{1,-} \\ D_{1,+} & 0\end{array}\right]$ is given by

$$
D_{1, \pm}=\mathrm{M} \pm \frac{\partial}{\partial r}
$$

where $\mathrm{M}$ still denotes multiplication by the input of the $\mathbb{R}$-component. Recall from Remark 4.6 that we can replace $D_{1}$ by $\frac{1}{2 \pi} \cdot D_{\lambda}$ for any $\lambda>0$, so for the best final results, we will choose $\lambda=2 \pi b>0$ :

$$
D_{\mathcal{H}}:=\frac{1}{2 \pi} \cdot D_{2 \pi b}=\left[\begin{array}{cc}
0 & b \mathrm{M}-\frac{1}{2 \pi} \frac{\partial}{\partial r} \\
b \mathrm{M}+\frac{1}{2 \pi} \frac{\partial}{\partial r} & 0
\end{array}\right] .
$$

Before we can state the main theorem of this section, we need some notation.

Definition 6.2. For a smooth function $F$ on $\mathbb{Z} \times \mathbb{T} \times \mathbb{R}^{n}$, any $N \in \mathbb{N}_{0}$, and $\alpha$ an $n$-multi-index, define the semi-norm

$$
\|F\|_{(N, \alpha)}^{\mathcal{S}_{n}}:=\sup \left\{\left(\|(k, \vec{x})\|_{N}^{N}+1\right)\left|\frac{\partial^{\alpha} \Phi}{\partial \vec{x}^{\alpha}}(k,[v], \vec{x})\right|:(k,[v], \vec{x}) \in \mathbb{Z} \times \mathbb{T} \times \mathbb{R}^{n}\right\}
$$

where $\frac{\partial^{\alpha}}{\partial \vec{x}^{\alpha}}$ is differentiation with respect to the $\mathbb{R}$-components. If $\|F\|_{(N, \alpha)}^{\mathcal{S}_{n}}$ is finite for every choice of $N$ and $\alpha$, then $F$ is called a Schwartz-Bruhat function. We will denote the locally convex space consisting of such $F$ by $\mathcal{S}_{n}$.

Remark 6.3. While it is possible to define a larger family of semi-norms by including differentiation in the $\mathbb{T}$-direction, the above seminorms are sufficient for our goals.

Definition 6.4. For functions on $\mathbb{Z} \times \mathbb{T} \times \mathbb{R}^{n}$, let $\mathrm{M}_{i}^{\mathbb{R}}$ be the operator of multiplication by the input of the $i^{\text {th }} \mathbb{R}$-component, and $\partial_{i}$ differentiation with respect to the $i^{\text {th }}$ $\mathbb{R}$-component. Let $\mathrm{M}^{\mathbb{Z}}$ be the operator of multiplication by the input of the $\mathbb{Z}$ component.

Note that all of these operators map $\mathcal{S}_{n}$ back into itself. We can now state the theorem:

Theorem 6.5. Let $\mathcal{R}^{ \pm}$be the completion of the right- $\mathfrak{A} \odot \mathfrak{A}$ pre-Hilbert module $R^{\infty}:=\mathcal{S}_{2}$ whose structure is defined by:

$$
\begin{aligned}
& \left(F \cdot \mathcal{R}\left(V^{l_{1}} U^{k_{1}} \otimes V^{l_{2}} U^{k_{2}}\right)\right)(k,[x], r, s) \\
& =\lambda^{l_{1}\left(k+k_{1}\right)+l_{2} k_{2}} \mathrm{e}^{2 \pi i x\left(l_{1}+l_{2}\right)} \mathrm{e}^{2 \pi i\left(l_{2} r-k_{2} s\right)} F\left(k-k_{2}+k_{1},\left[x+k_{2} \theta\right], r, s\right)
\end{aligned}
$$

and

$$
\begin{aligned}
& \left\langle F_{1} \mid F_{2}\right\rangle^{\mathcal{R}}\left(l_{1},[v], l_{2},[w]\right) \\
& =\sum_{k_{1}, k_{2} \in \mathbb{Z}} \int_{t} e^{2 \pi i t l_{2}} \overline{F_{1}}\left(k_{1},\left[v-k_{1} \theta\right], k_{2}+k_{1} \theta-v+w, t\right) \\
& \quad F_{2}\left(k_{1}+l_{2}-l_{1},\left[v-\left(k_{1}+l_{2}\right) \theta\right], k_{2}+k_{1} \theta-v+w, t\right) \mathrm{d} t .
\end{aligned}
$$

Let $\mathcal{R}:=\mathcal{R}^{+} \oplus \mathcal{R}^{-}$be standard evenly graded and define

$$
d_{\mathcal{R}}:=\left[\begin{array}{cc}
0 & d_{\mathcal{R},-} \\
d_{\mathcal{R},+} & 0
\end{array}\right] \text { where } d_{\mathcal{R}, \pm}:=\mathrm{M}_{1}^{\mathbb{R}} \mp i \mathrm{M}_{2}^{\mathbb{R}} \text { with } \operatorname{Dom}\left(d_{\mathcal{R}, \pm}\right):=\mathcal{S}_{2} .
$$

Then $\left(\mathcal{R}, d_{\mathcal{R}}\right)$ is a Kasparov cycle and represents $\widehat{\Delta}_{\theta}$. In particular, $\widehat{\Delta}_{\theta}$ does not depend on the choice of $b \in \mathbb{Z}^{\times}$. 
To prove this, we will make use of the following:

Theorem 6.6 (Special case of [16, Theorem 7.4]). Let $\mathcal{E}_{b}:=\mathcal{L}_{b} \otimes_{A_{\theta}^{\otimes 2}}\left(A_{\theta} \otimes \mathcal{H}_{-b}\right)$ for $b>0$, and suppose we have

(1) an odd, self-adjoint, regular operator $D_{\mathcal{E}}: \operatorname{Dom}\left(D_{\mathcal{E}}\right) \rightarrow \mathcal{E}_{b}$ so that

(2) $\left(0, D_{\mathcal{E}}\right)$ is a weakly anticommuting pair, and

(3) a dense $\mathfrak{A} \odot \mathfrak{A}$-submodule $\mathcal{X} \subseteq \mathcal{L}_{b}$ for which the algebraic tensor product $\mathcal{X} \odot_{\mathfrak{A} \odot \mathfrak{A}} \operatorname{Dom}\left(1_{A_{\theta}} \otimes D_{\mathcal{H}}\right)$ is a core for $D_{\mathcal{E}}$ such that

(4) for all $\Phi \in \mathcal{X}$, both operators $\eta \mapsto D_{\mathcal{E}, \pm}(\Phi \otimes \eta)-\Phi \otimes\left(1_{A_{\theta}} \otimes D_{\mathcal{H}, \pm}\right)(\eta)$ with domain $\operatorname{Dom}\left(1_{A_{\theta}} \otimes D_{\mathcal{H}, \pm}\right)$ extend to adjointable operators $A_{\theta} \otimes \mathcal{H}_{-b}^{ \pm} \rightarrow \mathcal{E}_{b}^{ \pm}$.

Then $\left(\mathcal{E}_{b}, D_{\mathcal{E}}\right)$ is a Kasparov cycle and represents $\widehat{\Delta}_{\theta}$.

Note that Item (2) is actually true no matter what self-adjoint regular operator $D_{\mathcal{E}}$ is chosen.

The remainder of this section is structured as follows: First, we find a description of $\mathcal{E}_{b}^{ \pm}$as a completion, called $\mathcal{P}_{b}^{ \pm}$, of $C_{c}\left(\mathbb{Z} \times \mathbb{T} \times \mathbb{R}^{2}\right)$. We will then prove that $\mathcal{E}_{b}^{ \pm}$contains $\mathcal{S}_{2}$, Schwartz-Bruhat functions on $\mathbb{Z} \times \mathbb{T} \times \mathbb{R}^{2}$, and explicitly describe the module structure of this subspace. Using a unitary operator, we simplify $\mathcal{E}_{b}$ to the module $\mathcal{R}$ from Theorem 6.5. On this easier module, we study the two unbounded operators $d_{\mathcal{R}, \pm}: \mathcal{R}^{ \pm} \rightarrow \mathcal{R}^{\mp}$ to then induce them to unbounded operators $D_{\mathcal{E}, \pm}: \mathcal{E}_{b}^{ \pm} \rightarrow \mathcal{E}_{b}^{\mp}$. Finally, we will show that the off-diagonal operator $D_{\mathcal{E}}$, built in the usual way out of $D_{\mathcal{E}, \pm}$, makes $\mathcal{E}_{b}$ a representative of $\widehat{\Delta}_{\theta}$. This will prove Theorem 6.5

Proposition 6.1 (The balancing). The module $\mathcal{E}_{b}^{ \pm}=\mathcal{L}_{b} \otimes_{A_{\theta}^{\otimes 2}}\left(A_{\theta} \otimes \mathcal{H}_{-b}^{ \pm}\right)$underlying $\widehat{\Delta}_{\theta}$ has a copy of $P^{\infty}:=C_{c}^{\infty}(\mathbb{Z} \times \mathbb{T} \times \mathbb{R}) \odot C_{c}^{\infty}(\mathbb{R})$ as a dense subspace via the following map:

$$
\begin{gathered}
\iota_{0}: \quad P^{\infty} \longrightarrow \mathcal{L}_{b} \otimes_{A_{\theta}^{\otimes 2}}\left(A_{\theta} \otimes \mathcal{H}_{-b}^{ \pm}\right) \\
\Phi \odot \psi \longmapsto \Phi \otimes\left(1_{A_{\theta}} \otimes \varepsilon_{0} \otimes z^{0} \otimes \psi\right)
\end{gathered}
$$

The proof is routine.

Lemma 6.7. The space $P^{\infty}=C_{c}^{\infty}(\mathbb{Z} \times \mathbb{T} \times \mathbb{R}) \odot C_{c}^{\infty}(\mathbb{R})$ inherits the following structure of a pre-Hilbert right-module from $\mathcal{E}_{b}^{ \pm}$via $\iota_{0}$ (the map in Lemma 6.1): the pre-inner product with values in $C_{c}^{\infty}(\mathcal{A})$ is given for $F_{i} \in P^{\infty}$ by

$$
\begin{aligned}
& \left\langle F_{1} \mid F_{2}\right\rangle^{\mathcal{P}}\left(l_{1},[v], l_{2},[w]\right) \\
& =\sum_{k_{1}, k_{2} \in \mathbb{Z}} \int_{\mathbb{R}} \overline{F_{1}}\left(k_{1},[v], \frac{k_{2}+k_{1} \theta-v+w}{b}-r, r\right) \\
& \quad F_{2}\left(k_{1}+l_{2}-l_{1},\left[v-l_{1} \theta\right], \frac{k_{2}+k_{1} \theta-v+w}{b}-r+l_{1}, r-l_{2}\right) \mathrm{d} r .
\end{aligned}
$$

The right action of an element $\xi \in \mathfrak{A} \odot \mathfrak{A}$ on $F \in P^{\infty}$ is given by:

$$
\begin{aligned}
& (F \cdot \mathcal{P} \xi)(k,[v], r, s)=\sum_{k_{1}, k_{2} \in \mathbb{Z}} F\left(k-k_{2}+k_{1},\left[v+k_{1} \theta\right], r-k_{1}, s+k_{2}\right) \\
& \cdot \xi\left(k_{1},\left[v+k_{1} \theta\right], k_{2},\left[v+b(r+s)+\left(k_{2}-k\right) \theta\right]\right) .
\end{aligned}
$$

The proof is straightforward.

Remark 6.8. If we let $\mathcal{P}_{b}^{ \pm}$be the completion of $P^{\infty}$ with respect to the above inner product, then $\iota_{0}$ extends, by construction, to a unitary $\mathcal{P}_{b}^{ \pm} \cong \mathcal{E}_{b}^{ \pm}$. 
The next goal is to prove the that $\mathcal{E}_{b}$ contains functions of Schwartz decay.

Proposition 6.2. The injective linear map $\iota_{0}: P^{\infty}=C_{c}^{\infty}(\mathbb{Z} \times \mathbb{T} \times \mathbb{R}) \odot C_{c}^{\infty}(\mathbb{R}) \rightarrow \mathcal{E}_{b}^{ \pm}$ from Lemma 6.1 extends to an injective linear map $\iota: \mathcal{S}_{2} \rightarrow \mathcal{E}_{b}^{ \pm}$. Moreover, the image of $\mathcal{S}_{2}$ is a right-A $\odot \mathfrak{A}$ pre-Hilbert submodule of $\mathcal{E}_{b}^{ \pm}$. The module structure on $\mathcal{S}_{2}$ induced by $\iota$ is given by the same formulas as on $P^{\infty}$.

Corollary 6.9. The completion $\mathcal{P}_{b}^{ \pm}$of $P^{\infty}$ has $\mathcal{S}_{2}$ as a dense subspace.

The main tool needed for the proof of Proposition 6.2 (see page 284) is the following result, proved using some estimates of quadruple series of rapid decay, and its corollaries.

Lemma 6.10. For any integer $N \geq 6$, there exists a finite number $\mu(N) \geq 0$ with the following property: If $F_{1}, F_{2} \in \mathcal{S}_{2}$, then for all $M, N \geq 6$,

$$
\left\|\left\langle F_{1} \mid F_{2}\right\rangle\right\|_{I} \leq \mu(M) \cdot\left\|F_{1}\right\|_{(M, 0)}^{\mathcal{S}_{2}} \cdot \mu(N) \cdot\left\|F_{2}\right\|_{(N, 0)}^{\mathcal{S}_{2}},
$$

where we define the inner product of two Schwartz-Bruhat functions by the same formula as Equation (6.5).

The interested reader can find a proof of the lemma and of the following in the first-named author's PhD thesis ([5] Lemma 7.2.5 ff.]). For a definition of the $I$ norm, see [23]. Note that the above, in particular, implies that $\left\langle F_{1} \mid F_{2}\right\rangle$ is indeed a function on $\mathcal{A}$ (i.e., that it takes finite values). With this tool, one proves the following:

Lemma 6.11. If $F_{n} \in P^{\infty}=C_{c}^{\infty}(\mathbb{Z} \times \mathbb{T} \times \mathbb{R}) \odot C_{c}^{\infty}(\mathbb{R})$ converges to $F \in \mathcal{S}_{2}$ with respect to $\|\cdot\|_{(M, 0)}^{\mathcal{S}_{2}}$, and $G_{n} \in P^{\infty}$ to $G \in \mathcal{S}_{2}$ in $\|\cdot\|_{(N, 0)}^{\mathcal{S}_{2}}$ for some $M, N \geq 6$, then $\left\langle F_{n} \mid G_{n}\right\rangle^{\mathcal{P}}$ converges to $\langle F \mid G\rangle^{\mathcal{S}}$ in $C^{*}(\mathcal{A})$. Consequently, the function $\langle F \mid G\rangle^{\mathcal{S}}$ is an element of $C^{*}(\mathcal{A})=A_{\theta} \otimes A_{\theta}$.

Using the fact that the $I$-norm dominates the $\mathrm{C}^{*}$-norm (see 23 , Chapter II, Proposition 4.2(ii)]), we conclude:

Corollary 6.12. For any integers $M, N \geq 6$ and with $\mu(N)$ as in Lemma 6.10, we have for all $F_{j}$ in $\mathcal{S}_{2}$ :

$$
\left\|\left\langle F_{1} \mid F_{2}\right\rangle^{\mathcal{S}}\right\|_{C^{*}(\mathcal{A})} \leq \mu(M) \cdot\left\|F_{1}\right\|_{(M, 0)}^{\mathcal{S}_{2}} \cdot \mu(N) \cdot\left\|F_{2}\right\|_{(N, 0)}^{\mathcal{S}_{2}} \cdot
$$

In particular, if $F \in P^{\infty}$, then $\left\|\iota_{0}(F)\right\|_{\mathcal{E}_{b}} \leq \mu(N) \cdot\|F\|_{(N, 0)}^{\mathcal{S}_{2}}$.

Using the fact that the $\mathrm{C}^{*}$-norm dominates the uniform-norm (see [23. Chapter II, Proposition 4.1(i)]), we also conclude:

Corollary 6.13. For $F$ in $\mathcal{S}_{2}$, we have

$$
\left\|\langle F \mid F\rangle^{\mathcal{S}}\right\|_{C^{*}(\mathcal{A})} \geq \sup \left\{\int_{\mathbb{R}}|F|^{2}(k,[v], s-r, r) \mathrm{d} r:[v] \in \mathbb{T}, k \in \mathbb{Z}, s \in \mathbb{R}\right\} .
$$

Lemma 6.14. If $F$ in $\mathcal{S}_{2}$ and $\xi$ in $\mathfrak{A} \odot \mathfrak{A}$, and if $F_{\mathcal{S}_{2}} \xi$ is defined by the same formula as Equation (6.6), then $F_{\mathcal{S}_{2}} \xi$ is an element of $\mathcal{S}_{2}$. Moreover, if $F_{n} \in$ $P^{\infty}=C_{c}^{\infty}(\mathbb{Z} \times \mathbb{T} \times \mathbb{R}) \odot C_{c}^{\infty}(\mathbb{R})$ converges to $F$ in $\mathcal{S}_{2}$, then $F_{n} \cdot \mathcal{P} \xi$ converges to $F \cdot \mathcal{S}_{2} \xi$ in $\mathcal{S}_{2}$. 
Proof of Proposition 6.2. Take any $F \in \mathcal{S}_{2}$ and let $F_{n} \in P^{\infty}=C_{c}^{\infty}(\mathbb{Z} \times \mathbb{T} \times \mathbb{R}) \odot$ $C_{c}^{\infty}(\mathbb{R})$ be a sequence which converges to $F$ in $\mathcal{S}_{2}$; in particular, for any $\epsilon>0$ and for $n, m$ sufficiently large,

$$
\left\|F_{n}-F_{m}\right\|_{(4,0)}^{\mathcal{S}_{2}} \leq\left\|F_{n}-F\right\|_{(4,0)}^{\mathcal{S}_{2}}+\left\|F-F_{m}\right\|_{(4,0)}^{\mathcal{S}_{2}}<\epsilon .
$$

By Corollary 6.12 the sequence $\left(\iota_{0}\left(F_{n}\right)\right)_{n}$ is therefore Cauchy in $\mathcal{E}_{b}^{ \pm}$and hence converges; let $\iota(F)$ denote the limit in $\mathcal{E}_{b}^{ \pm}$. Note that, if $\lim _{n}^{\mathcal{S}} F_{n}=F=0$, then $\lim _{n}^{\mathcal{E}} \iota_{0}\left(F_{n}\right)=0$ by the same corollary, so $\iota(F)$ does not depend on the chosen sequence in $P^{\infty}$ and for $F \in P$, we have $\iota(F)=\iota_{0}(F)$. Using Corollary 6.12 yet again, we get for any integer $N \geq 6$ :

$$
\begin{aligned}
\left\|\langle\iota(F) \mid \iota(F)\rangle^{\mathcal{E}}\right\|_{C^{*}(\mathcal{A})}^{\frac{1}{2}} & =\|\iota(F)\|_{\mathcal{E}_{b}}=\lim _{n \rightarrow \infty}\left\|\iota_{0}\left(F_{n}\right)\right\|_{\mathcal{E}_{b}} \\
& \leq \lim _{n \rightarrow \infty}\left(\left\|F_{n}\right\|_{(N, 0)}^{\mathcal{S}_{2}} \cdot \mu(N)\right)=\|F\|_{(N, 0)}^{\mathcal{S}_{2}} \cdot \mu(N) .
\end{aligned}
$$

To check that the extended map $\iota$ is injective, note first that there exists a constant $K$ such that for any $F \in \mathcal{S}_{2}$ and any $N \geq 2$ :

$$
K \cdot\left(\|F\|_{(N, 0)}^{\mathcal{S}_{2}}\right)^{2} \geq \sup \left\{\int_{\mathbb{R}}|F|^{2}(k,[v], s-r, r) \mathrm{d} r:[v] \in \mathbb{T}, k \in \mathbb{Z}, s \in \mathbb{R}\right\} .
$$

Using Lemma 6.13 this implies

$$
\|\iota(F)\|_{\mathcal{E}_{b}}^{2} \geq \sup \left\{\int_{\mathbb{R}}|F|^{2}(k,[v], s-r, r) \mathrm{d} r:[v] \in \mathbb{T}, k \in \mathbb{Z}, s \in \mathbb{R}\right\},
$$

i.e. if $\|\iota(F)\|_{\mathcal{E}_{b}}=0$, then $F \equiv 0$, so $\iota$ is injective. Some more estimates with Lemma 6.11 and Corollary 6.12 show

$$
\iota(F) \cdot \mathcal{E}_{b} \xi=\iota\left(F \cdot \mathcal{S}_{2} \xi\right) \text { and }\langle F \mid G\rangle^{\mathcal{S}}=\langle\iota(F) \mid \iota(G)\rangle^{\mathcal{E}}
$$

where $\xi \in \mathfrak{A} \odot \mathfrak{A}$, which concludes our proof.

Remark 6.15. One proves mutatis mutandis that the inclusions $C_{c}^{\infty}(\mathbb{Z} \times \mathbb{T} \times \mathbb{R}) \subseteq \mathcal{L}_{b}$ and $C_{c}^{\infty}(\mathbb{Z} \times \mathbb{T} \times \mathbb{R}) \subseteq \mathcal{H}_{b}^{ \pm}$(which are dense by Corollary [5.9) extend to injective linear maps $\mathcal{S}_{1} \rightarrow \mathcal{L}_{b}$ resp. $\mathcal{S}_{1} \rightarrow \mathcal{H}_{b}^{ \pm}$, and that the respective right pre-Hilbert module formulas on $C_{c}^{\infty}(\mathbb{Z} \times \mathbb{T} \times \mathbb{R})$ are still valid for elements in $\mathcal{S}_{1}$. Fully analogously to the map $\iota_{0}: P^{\infty}=C_{c}^{\infty}(\mathbb{Z} \times \mathbb{T} \times \mathbb{R}) \odot C_{c}^{\infty}(\mathbb{R}) \rightarrow \mathcal{E}_{b}^{ \pm}$from Lemma 6.1, we could therefore have defined the map

$$
\begin{aligned}
\iota^{\prime}: & \mathcal{S}_{1} \odot \mathcal{S}(\mathbb{R}) \longrightarrow \mathcal{L}_{b} \otimes_{A_{\theta}^{\otimes 2}}\left(A_{\theta} \otimes \mathcal{H}_{-b}^{ \pm}\right)=\mathcal{E}_{b}^{ \pm} \\
& \Phi \odot \psi \longmapsto \Phi \otimes\left(1_{A_{\theta}} \otimes \varepsilon_{0} \otimes z^{0} \otimes \psi\right),
\end{aligned}
$$

which clearly also has dense image. By construction, $\iota^{\prime}$ and $\iota_{0}$ give rise to the same extension, namely the injective linear map $\iota: \mathcal{S}_{2} \rightarrow \mathcal{E}_{b}^{ \pm}$from Proposition 6.2.

Now that we have simplified $\mathcal{E}_{b}$, we would like to show that it is unitarily equivalent to the module $\mathcal{R}$ from Theorem 6.5

Definition 6.16. Let

$$
\begin{aligned}
& \chi: \mathcal{S}_{2} \rightarrow \mathcal{S}_{2}, \chi(F)(k,[x], r, s):=\int_{t} F(k,[x], r, t) \mathrm{e}^{-2 \pi i t s} \mathrm{~d} t, \\
& \text { and } \quad \Gamma: \mathcal{S}_{2} \rightarrow \mathcal{S}_{2}, \quad \Gamma(F)(k,[x], r, s):=F(k,[x-k \theta], b(r+s+k), s)
\end{aligned}
$$


with inverses given by

$$
\begin{aligned}
\chi^{-1}(F)(k,[x], r, s) & :=\int_{q} F(k,[x], r, q) \mathrm{e}^{2 \pi i q s} \mathrm{~d} q \\
\text { and } \quad \Gamma^{-1}(F)(k,[x], r, s) & :=F\left(k,[x+k \theta], \frac{r}{b}-s-k, s\right) .
\end{aligned}
$$

And define

$$
\begin{aligned}
\mathcal{S}_{2} & \rightarrow \mathcal{S}_{2} \\
\Xi(F)(k,[x], r, s) & =\int_{t} F(k,[x-k \theta], b(r+s+k), t) \mathrm{e}^{-2 \pi i t s} \mathrm{~d} t .
\end{aligned}
$$

with inverse

$$
\Xi^{-1}(F)(k,[x], r, s)=\int_{q} F\left(k,[x+k \theta], \frac{r}{b}-q-k, q\right) \mathrm{e}^{2 \pi i q s} \mathrm{~d} q
$$

Theorem 6.17. The map $\Xi$ extends to a unitary from $\mathcal{R}^{ \pm}$, the completion of the pre-Hilbert module $R^{\infty}$ defined in Theorem [6.5, to $\mathcal{P}_{b}^{ \pm}$, the completion of the pre-Hilbert module $P^{\infty}$ defined in Lemma 6.7.

Proof. A direct computation shows that the linear map $\Xi: R^{\infty} \rightarrow \mathcal{S}_{2} \subseteq \mathcal{P}_{b}^{ \pm}$preserves the pre-inner product and right $\mathfrak{A} \odot \mathfrak{A}$-module structure on $\mathcal{S}_{2}=R^{\infty} \subseteq \mathcal{R}^{ \pm}$. As $\Xi$ is a bijection $\mathcal{S}_{2} \rightarrow \mathcal{S}_{2}$, and as $\mathcal{S}_{2}$ is dense in both $\mathcal{R}^{ \pm}$and $\mathcal{P}_{b}^{ \pm}$by definition, $\Xi$ extends to a unitary $\mathcal{R}^{ \pm} \cong \mathcal{P}_{b}^{ \pm}$.

Corollary 6.18. The map $\iota \circ \Xi: R^{\infty} \rightarrow \mathcal{E}_{b}^{ \pm}=\mathcal{L}_{b} \otimes_{A_{\theta}^{\otimes 2}}\left(A_{\theta} \otimes \mathcal{H}_{b}^{ \pm}\right)$extends to a unitary $\mathcal{R}^{ \pm} \cong \mathcal{E}_{b}^{ \pm}$, where $\iota$ is the injective linear map from Proposition 6.2 .

We now turn to the operator.

Lemma 6.19. The closure of the operator $d_{\mathcal{R}}$ from Equation (6.4) is self-adjoint and regular.

Proof. Because $\mathrm{M}_{1}^{\mathbb{R}}$ and $\mathrm{M}_{2}^{\mathbb{R}}$ are obviously symmetric in view of the inner product defined on $\mathcal{R}^{ \pm}$(see Equation (6.3) ), so is $d_{\mathcal{R}}$. Since the domain of $d_{\mathcal{R}}$ is the dense set $\mathcal{S}_{2}$, it thus suffices to check that $d_{\mathcal{R}} \pm i$ has dense range. For any given $\psi_{1}, \psi_{2} \in \mathcal{S}_{2}$, define

$$
\begin{aligned}
& \phi_{1}(k,[x], r, s):=\frac{(r+i s) \cdot \psi_{2}(k,[x], r, s) \mp i \psi_{1}(k,[x], r, s)}{1+s^{2}+r^{2}}, \text { and } \\
& \phi_{2}(k,[x], r, s):=\frac{(r-i s) \cdot \psi_{1}(k,[x], r, s) \mp i \psi_{2}(k,[x], r, s)}{1+s^{2}+r^{2}} .
\end{aligned}
$$

These functions lie in the domain of our operator $d_{\mathcal{R}}$ and satisfy $\left(d_{\mathcal{R}} \pm i\right)\left(\phi_{1} \oplus \phi_{2}\right)=$ $\psi_{1} \oplus \psi_{2}$, so the range of $d_{\mathcal{R}} \pm i$ contains $\mathcal{S}_{2}^{\oplus 2}$ and is hence dense.

Corollary 6.20 (Using Theorem 6.17). On $\mathcal{P}_{b}$, the closure of the operator

$$
d_{\mathcal{P}}:=\left[\begin{array}{cc}
0 & d_{\mathcal{P},-} \\
d_{\mathcal{P},+} & 0
\end{array}\right] \text { where } d_{\mathcal{P}, \pm}:=\Xi \circ d_{\mathcal{R}, \pm} \circ \Xi^{-1} \text { with } \operatorname{Dom}\left(d_{\mathcal{P}, \pm}\right):=\mathcal{S}_{2} \subseteq \mathcal{P}_{b}^{ \pm},
$$

is self-adjoint and regular. 
Note that the definition of $d_{\mathcal{P}, \pm}$ indeed makes sense since $d_{\mathcal{R}, \pm}$ maps its domain $\mathcal{S}_{2}$ back into itself. A direct computation shows:

Lemma 6.21. We have

$$
\Xi \circ \mathrm{M}_{1}^{\mathbb{R}} \circ \Xi^{-1}=b\left(\mathrm{M}_{1}^{\mathbb{R}}+\mathrm{M}^{\mathbb{Z}}+\mathrm{M}_{2}^{\mathbb{R}}\right) \quad \text { and } \quad \Xi \circ \mathrm{M}_{2}^{\mathbb{R}} \circ \Xi^{-1}=\frac{i}{2 \pi}\left(\partial_{2}-\partial_{1}\right) \text {, }
$$

where $\Xi: \mathcal{S}_{2} \rightarrow \mathcal{S}_{2}$ is the map defined in Definition 6.16. In particular,

$$
d_{\mathcal{P}, \pm}=\left[b\left(\mathbf{M}_{1}^{\mathbb{R}}+\mathbf{M}^{\mathbb{Z}}\right) \mp \frac{1}{2 \pi} \partial_{1}\right]+\left[b \mathbf{M}_{2}^{\mathbb{R}} \pm \frac{1}{2 \pi} \partial_{2}\right] .
$$

We should remark that we have written $d_{\mathcal{P}, \pm}$ in such a way because the $\mathbb{Z}$ and the first $\mathbb{R}$-component both arose from the copy of $\mathcal{L}_{b}$ inside of $\mathcal{E}_{b}^{ \pm}$, while the second $\mathbb{R}$-component arose from the copy of $\mathcal{H}_{-b}^{ \pm} ; c f$. the map $\iota_{0}$ in Lemma 6.1 with extension $\iota$ constructed in Proposition 6.2

As $\iota$ is an injective map and as $d_{\mathcal{P}, \pm}$ maps its domain $\mathcal{S}_{2}$ back into itself, it makes sense to define the following operator on $\mathcal{E}_{b}$ :

$$
D_{\mathcal{E}}:=\left[\begin{array}{cc}
0 & D_{\mathcal{E},-} \\
D_{\mathcal{E},+} & 0
\end{array}\right] \text { where } D_{\mathcal{E}, \pm}:=\iota \circ d_{\mathcal{P}, \pm} \circ \iota^{-1} \text { with } \operatorname{Dom}\left(D_{\mathcal{E}, \pm}\right):=\operatorname{ran}(\iota) \subseteq \mathcal{E}_{b}^{ \pm} .
$$

Note that $D_{\mathcal{E}}$ is densely defined according to Lemma 6.1 Moreover, its closure is self-adjoint and regular because the closure of $d_{\mathcal{P}}$ is by Corollary 6.20

Recall that we chose

$$
D_{\mathcal{H}, \pm}=b \mathrm{M}^{\mathbb{R}} \pm \frac{1}{2 \pi} \frac{\partial}{\partial r}
$$

in Equation (6.1). Its domain can be chosen to be $\operatorname{Dom}\left(D_{\mathcal{H}, \pm}\right):=\mathcal{S}_{1} \subseteq \mathcal{H}_{-b}^{ \pm}$thanks to Remark 6.15]

Lemma 6.22. Let

$$
D_{\mathcal{L}, \pm}:=b\left(\mathrm{M}^{\mathbb{R}}+\mathrm{M}^{\mathbb{Z}}\right) \mp \frac{1}{2 \pi} \frac{\partial}{\partial r} \text { with } \operatorname{Dom}\left(D_{\mathcal{L}, \pm}\right):=\mathcal{S}_{1} \subseteq \mathcal{L}_{b}
$$

On the image under $\iota$ of the subspace $\mathcal{S}_{1} \odot \mathcal{S}(\mathbb{R})$ of $\mathcal{S}_{2}$, we have

$$
D_{\mathcal{E}, \pm}=D_{\mathcal{L}, \pm_{ \pm}}{A_{\theta}^{\otimes 2}}\left(1_{A_{\theta} \otimes \mathcal{H}_{-b}^{ \pm}}\right)+1_{\mathcal{L}_{b}} \otimes_{A_{\theta}^{\otimes 2}}\left(1_{A_{\theta}} \otimes D_{\mathcal{H}, \pm}\right) .
$$

In the above, we have written $\otimes_{A_{\theta}^{\otimes 2}}$ (instead of the more customary $\otimes$ ) to emphasize that $\mathcal{E}_{b}^{ \pm}=\mathcal{L}_{b} \otimes_{A_{\theta}^{\otimes 2}}\left(A_{\theta} \otimes \mathcal{H}_{-b}^{ \pm}\right)$is the balanced tensor product (so it is not obvious a priori that the above operator is well-defined).

Proof. Recall that $\iota$ is the extension of the map

$$
\iota_{0}: \quad C_{c}^{\infty}(\mathbb{Z} \times \mathbb{T} \times \mathbb{R}) \odot C_{c}^{\infty}(\mathbb{R}) \rightarrow \mathcal{L}_{b} \otimes_{A_{\theta}^{\otimes 2}}\left(A_{\theta} \otimes \mathcal{H}_{-b}^{ \pm}\right)=\mathcal{E}_{b}^{ \pm}
$$

from Lemma 6.1 to all of $\mathcal{S}_{2}$. In particular, on the subspace $\mathcal{S}_{1} \odot \mathcal{S}(\mathbb{R}), \iota$ is given by the exact same formula as $\iota_{0}$, namely

$$
\iota(\Phi \odot \psi)=\Phi \otimes\left(1_{A_{\theta}} \otimes \varepsilon_{0} \otimes z^{0} \otimes \psi\right) .
$$

It is then obvious that $D_{\mathcal{E}, \pm}$, defined as $\iota \circ d_{\mathcal{P},{ }_{ \pm}} \circ \iota^{-1}$ with

$$
d_{\mathcal{P}, \pm}=\left[b\left(\mathrm{M}_{1}^{\mathbb{R}}+\mathrm{M}^{\mathbb{Z}}\right) \mp \frac{1}{2 \pi} \partial_{1}\right]+\left[b \mathrm{M}_{2}^{\mathbb{R}} \pm \frac{1}{2 \pi} \partial_{2}\right]
$$

computed in Lemma 6.21 is indeed as claimed. 
Lemma 6.23. The operator $d_{\mathcal{P}, \pm}$ leaves the subspace $\mathcal{S}_{1} \odot \mathcal{S}(\mathbb{R})$ of $\mathcal{S}_{2}$ invariant. Moreover, $\mathcal{S}_{1} \odot \mathcal{S}(\mathbb{R})$ is a core for $d_{\mathcal{P}, \pm}$.

The invariance is obvious, and the proof regarding the core requires only an application of Corollary 6.12 (in fact, one proves that any subspace of $\mathcal{S}_{2}=\operatorname{Dom}\left(d_{\mathcal{P}, \pm}\right.$ ) which is dense with respect to the family of seminorms on $\mathcal{S}_{2}$, is a core for $\left.d_{\mathcal{P}, \pm}\right)$. Since $D_{\mathcal{E}, \pm}:=\iota \circ d_{\mathcal{P}, \pm} \circ \iota^{-1}$ (see Equation (6.9) ), a consequence is that Item (3) holds for $\left(\mathcal{E}_{b}, D_{\mathcal{E}}\right)$ :

Corollary 6.24. The dense $\mathfrak{A} \odot \mathfrak{A}$-submodule $\operatorname{Dom}\left(D_{\mathcal{L}}\right)=\mathcal{S}_{1} \subseteq \mathcal{L}_{b}$ makes $\mathcal{S}_{1} \odot_{\mathfrak{A} \odot \mathfrak{A}}$ $\operatorname{Dom}\left(1_{A_{\theta}} \otimes D_{\mathcal{H}}\right)$ a core for $D_{\mathcal{E}}$.

Item (4) holds as well for $\left(\mathcal{E}_{b}, D_{\mathcal{E}}\right)$ :

Lemma 6.25. For all $\Phi \in \operatorname{Dom}\left(D_{\mathcal{L}}\right)=\mathcal{S}_{1} \subseteq \mathcal{L}_{b}$, both operators $\eta \mapsto D_{\mathcal{E}, \pm}(\Phi \otimes \eta)-$ $\Phi \otimes\left(1_{A_{\theta}} \otimes D_{\mathcal{H}, \pm}\right)(\eta)$ with domain $\operatorname{Dom}\left(1_{A_{\theta}} \otimes D_{\mathcal{H}, \pm}\right)$ extend to adjointable operators $A_{\theta} \otimes \mathcal{H}_{-b}^{ \pm} \rightarrow \mathcal{E}_{b}^{ \pm}$.

Proof. For $\eta=a \otimes \Psi$ for $a \in \mathfrak{A}$ and $\Psi \in \mathcal{S}_{1} \subseteq \mathcal{H}_{-b}^{ \pm}$and $\Phi \in \mathcal{S}_{1} \subseteq \mathcal{L}_{b}$ :

$$
\begin{aligned}
& D_{\mathcal{E}, \pm}\left(\Phi \otimes_{A_{\theta}^{\otimes 2}} \eta\right)-\Phi \otimes_{A_{\theta}^{\otimes 2}}\left(1_{A_{\theta}} \otimes D_{\mathcal{H}, \pm}\right)(\eta) \\
& \quad=\left(D_{\mathcal{L}, \pm} \Phi\right) \otimes_{A_{\theta}^{\otimes 2}}(a \otimes \Psi)+\Phi \otimes_{A_{\theta}^{\otimes 2}}\left(a \otimes D_{\mathcal{H}, \pm}(\Psi)\right)-\Phi \otimes_{A_{\theta}^{\otimes 2}}\left(1_{A_{\theta}} \otimes D_{\mathcal{H}, \pm}\right)(\eta) \\
& =\left(D_{\mathcal{L}, \pm} \Phi\right) \otimes_{A_{\theta}^{\otimes 2}} \eta=T_{D_{\mathcal{L}, \pm} \Phi}(\eta) .
\end{aligned}
$$

We conclude for general $\eta$ that $D_{\mathcal{E}, \pm}\left(\Phi \otimes_{A_{\theta}^{\otimes 2}} \eta\right)-\Phi \otimes_{A_{\theta}^{\otimes 2}}\left(1_{A_{\theta}} \otimes D_{\mathcal{H}, \pm}\right)(\eta)=T_{D_{\mathcal{L}, \pm} \Phi}(\eta)$, so we have shown that the operator in question is extended by a creation operator, which is clearly adjointable.

Proposition 6.3. The pair $\left(\mathcal{E}_{b}, D_{\mathcal{E}}\right)$ is a Kasparov cycle and represents $\widehat{\Delta}_{\theta}$.

Proof. Recall that $\widehat{\Delta}_{\theta}$ was defined as $\left[\mathcal{L}_{b}\right] \otimes_{A_{\theta}^{\otimes 2}}\left(1_{A_{\theta}} \otimes x_{-b}\right)$. We have checked that the items in Theorem 6.6 are all satisfied:

As explained on page 286 the closure of $D_{\mathcal{E}}$ is self-adjoint and regular (i.e. Item (1) holds) because $D_{\mathcal{E}}$ is unitarily equivalent to the operator $d_{\mathcal{P}}$, whose closure is self-adjoint and regular by Corollary 6.20 . We explained that $\left(0, D_{\mathcal{E}}\right)$ is a weakly anticommuting pair (i.e. Item (2) holds), and in Lemma 6.24, we have proved that for the dense submodule $\mathcal{X}:=\mathcal{S}_{1}$ of $\mathcal{L}_{b}$, the algebraic tensor product $\mathcal{S}_{1} \odot \operatorname{Dom}\left(1_{A_{\theta}} \otimes\right.$ $\left.D_{\mathcal{H}}\right)$ is a core for $D_{\mathcal{E}}$ (i.e. Item $(3)$ holds). Lastly, in Lemma 6.25 we have shown that, for $\Phi \in \mathcal{X}$, the operator $D_{\mathcal{E}} T_{\Phi}-T_{\Phi}\left(1_{A_{\theta}} \otimes D_{\mathcal{H}}\right)$ has an adjointable extension, i.e. Item (4) holds as well.

Proof of Theorem 6.5. We have shown in Corollary 6.18 that $\mathcal{R}$ is unitarily equivalent to $\mathcal{E}_{b}$, and we have defined $D_{\mathcal{E}}$ exactly so that the unitary equivalence turns it into $d_{\mathcal{R}}$. The claim now follows from Proposition 6.3

\section{ACKnowledgments}

This research was carried out in the course of the first-named author's Ph.D. at the University of Victoria, and forms part of her thesis [5]. We would like to thank Marcelo Laca and Ian Putnam for their sage remarks and advice during the production of this paper, and the referees for their careful reading and helpful corrections. 


\section{REFERENCES}

[1] Berndt A. Brenken, Representations and automorphisms of the irrational rotation algebra, Pacific J. Math. 111 (1984), no. 2, 257-282. MR734854

[2] Alain Connes, Noncommutative geometry, Academic Press, Inc., San Diego, CA, 1994. MR 1303779

[3] Alain Connes, Gravity coupled with matter and the foundation of non-commutative geometry, Comm. Math. Phys. 182 (1996), no. 1, 155-176. MR1441908

[4] A. Connes and G. Skandalis, The longitudinal index theorem for foliations, Publ. Res. Inst. Math. Sci. 20 (1984), no. 6, 1139-1183, DOI 10.2977/prims/1195180375. MR775126

[5] A. Duwenig, Poincaré self-duality of $A_{\theta}, 2020$, URI: http://hdl.handle.net/1828/11678

[6] Siegfried Echterhoff, Heath Emerson, and Hyun Jeong Kim, KK-theoretic duality for proper twisted actions, Math. Ann. 340 (2008), no. 4, 839-873, DOI 10.1007/s00208-007-0171-6. MR2372740

[7] Heath Emerson, Lefschetz numbers for $C^{*}$-algebras, Canad. Math. Bull. 54 (2011), no. 1, 82-99, DOI 10.4153/CMB-2010-084-5. MR2797970

[8] Heath Emerson, Noncommutative Poincaré duality for boundary actions of hyperbolic groups, J. Reine Angew. Math. 564 (2003), 1-33, DOI 10.1515/crll.2003.090. MR2021032

[9] Heath Emerson, The class of a fibre in noncommutative geometry, J. Geom. Phys. 148 (2020), 103537, 37, DOI 10.1016/j.geomphys.2019.103537. MR4043062

[10] José M. Gracia-Bondía, Joseph C. Várilly, and Héctor Figueroa, Elements of noncommutative geometry, Birkhäuser Advanced Texts: Basler Lehrbücher. [Birkhäuser Advanced Texts: Basel Textbooks], Birkhäuser Boston, Inc., Boston, MA, 2001. MR.1789831

[11] Jens Kaad, On the unbounded picture of KK-theory, SIGMA Symmetry Integrability Geom. Methods Appl. 16 (2020), 082, 21 pages, DOI 10.3842/SIGMA.2020.082. MR 4137615

[12] Jerome Kaminker and Ian Putnam, K-theoretic duality of shifts of finite type, Comm. Math. Phys. 187 (1997), no. 3, 509-522, DOI 10.1007/s002200050147. MR1468312

[13] Jerome Kaminker, Ian F. Putnam, and Michael F. Whittaker, K-theoretic duality for hyperbolic dynamical systems, J. Reine Angew. Math. 730 (2017), 263-299, DOI 10.1515/crelle2014-0126. MR.3692021

[14] G. G. Kasparov, Equivariant KK-theory and the Novikov conjecture, Invent. Math. 91 (1988), no. 1, 147-201, DOI 10.1007/BF01404917. MR918241

[15] Dan Kucerovsky, The KK-product of unbounded modules, K-Theory 11 (1997), no. 1, 17-34, DOI 10.1023/A:1007751017966. MR:1435704

[16] Matthias Lesch and Bram Mesland, Sums of regular self-adjoint operators in Hilbert-C $C^{*}$ modules, J. Math. Anal. Appl. 472 (2019), no. 1, 947-980, DOI 10.1016/j.jmaa.2018.11.059. MR3906406

[17] Wolfgang Lück and Jonathan Rosenberg, Equivariant Euler characteristics and K-homology Euler classes for proper cocompact G-manifolds, Geom. Topol. 7 (2003), 569-613, DOI 10.2140/gt.2003.7.569. MR2026542

[18] Bram Mesland, Unbounded bivariant K-theory and correspondences in noncommutative geometry, J. Reine Angew. Math. 691 (2014), 101-172, DOI 10.1515/crelle-2012-0076. MR 3213549

[19] Ralf Meyer and Ryszard Nest, The Baum-Connes conjecture via localisation of categories, Topology 45 (2006), no. 2, 209-259, DOI 10.1016/j.top.2005.07.001. MR2193334

[20] Paul S. Muhly, Jean N. Renault, and Dana P. Williams, Equivalence and isomorphism for groupoid $C^{*}$-algebras, J. Operator Theory 17 (1987), no. 1, 3-22. MR873460

[21] Ian F. Putnam and Jack Spielberg, The structure of $C^{*}$-algebras associated with hyperbolic dynamical systems, J. Funct. Anal. 163 (1999), no. 2, 279-299, DOI 10.1006/jfan.1998.3379. MR.1680475

[22] Graham A. Niblo, Roger Plymen, and Nick Wright, Poincaré duality and Langlands duality for extended affine Weyl groups, Ann. K-Theory 3 (2018), no. 3, 491-522, DOI 10.2140/akt.2018.3.491. MR.3830200

[23] Jean Renault, A groupoid approach to $C^{*}$-algebras, Lecture Notes in Mathematics, vol. 793, Springer, Berlin, 1980. MR584266

[24] Lachlan MacDonald and Adam Rennie, The Godbillon-Vey invariant and equivariant KKtheory, Ann. K-Theory 5 (2020), no. 2, 249-294, DOI 10.2140/akt.2020.5.249. MR4113770 
[25] Adam Rennie, David Robertson, and Aidan Sims, Poincaré duality for Cuntz-Pimsner algebras, Adv. Math. 347 (2019), 1112-1172, DOI 10.1016/j.aim.2019.02.032. MR3924388

[26] John Roe, Elliptic operators, topology and asymptotic methods, 2nd ed., Pitman Research Notes in Mathematics Series, vol. 395, Longman, Harlow, 1998. MR1670907

[27] Yasuo Watatani, Toral automorphisms on irrational rotation algebras, Math. Japon. 26 (1981), no. 4, 479-484. MR634924

School of Mathematics and Applied Statistics, University of Wollongong, NorthFields Ave, Wollongong, NSW 2522, Australia

Email address: aduwenig@uow.edu.au

Department of Mathematics and Statistics, University of Victoria, P.O. Box 3045 STN CSC, Victoria, British Columbia, V8W 3P4 Canada

Email address: hemerson@math.uvic.ca 